\title{
Radial distribution of dust, stars, gas, and star-formation rate in DustPedia ${ }^{\star}$ face-on galaxies
}

\author{
V. Casasola ${ }^{1}$, L. P. Cassarà ${ }^{2}$, S. Bianchi ${ }^{1}$, S. Verstocken ${ }^{3}$, E. Xilouris ${ }^{2}$, L. Magrini ${ }^{1}$, M. W. L. Smith ${ }^{4}$, I. De Looze ${ }^{5}$ \\ M. Galametz ${ }^{6}$, S. C. Madden ${ }^{6}$, M. Baes ${ }^{3}$, C. Clark ${ }^{4}$, J. Davies ${ }^{4}$, P. De Vis ${ }^{7}$, R. Evans ${ }^{4}$, J. Fritz ${ }^{8}$, F. Galliano ${ }^{6}$, \\ A. P. Jones ${ }^{7}$, A. V. Mosenkov ${ }^{3}$, S. Viaene ${ }^{3,9}$, and N. Ysard ${ }^{7}$ \\ 1 INAF-Osservatorio Astrofisico di Arcetri, Largo E. Fermi 5, 50125 Florence, Italy \\ e-mail: casasola@arcetri.astro.it \\ 2 National Observatory of Athens, Institute for Astronomy, Astrophysics, Space Applications and Remote Sensing, \\ Ioannou Metaxa and Vasileos Pavlou, 15236 Athens, Greece \\ 3 Sterrenkundig Observatorium, Department of Physics and Astronomy, Universiteit Gent Krijgslaan 281 S9, 9000 Gent, Belgium \\ ${ }^{4}$ School of Physics and Astronomy, Cardiff University, The Parade, Cardiff CF24 3AA, UK \\ 5 Department of Physics and Astronomy, University College London, Gower Street, London WC1E 6BT, UK \\ ${ }^{6}$ Laboratoire AIM, CEA/DSM - CNRS - Université Paris Diderot, IRFU/Service d'Astrophysique, CEA Saclay, \\ 91191 Gif-sur-Yvette, France \\ 7 Institut d'Astrophysique Spatiale, CNRS, Univ. Paris-Sud, Université Paris-Saclay, Bât. 121, 91405, Orsay Cedex, France \\ 8 Instituto de Radioastronomía y Astrofísica, UNAM, Campus Morelia, A.P. 3-72, C.P. 58089, Mexico \\ 9 Centre for Astrophysics Research, University of Hertfordshire, College Lane, Hatfield AL10 9AB, UK
}

Received 22 April 2017 / Accepted 14 June 2017

\begin{abstract}
Aims. The purpose of this work is the characterization of the radial distribution of dust, stars, gas, and star-formation rate (SFR) in a sub-sample of 18 face-on spiral galaxies extracted from the DustPedia sample.

Methods. This study is performed by exploiting the multi-wavelength DustPedia database, from ultraviolet (UV) to sub-millimeter bands, in addition to molecular $\left({ }^{12} \mathrm{CO}\right)$ and atomic (HI) gas maps and metallicity abundance information available in the literature. We fitted the surface-brightness profiles of the tracers of dust and stars, the mass surface-density profiles of dust, stars, molecular gas, and total gas, and the SFR surface-density profiles with an exponential curve and derived their scale-lengths. We also developed a method to solve for the $\mathrm{CO}-$ to- $\mathrm{H}_{2}$ conversion factor $\left(\alpha_{\mathrm{CO}}\right)$ per galaxy by using dust- and gas-mass profiles.

Results. Although each galaxy has its own peculiar behavior, we identified a common trend of the exponential scale-lengths versus wavelength. On average, the scale-lengths normalized to the $B$-band $25 \mathrm{mag} / \operatorname{arcsec}^{2}$ radius decrease from UV to $70 \mu \mathrm{m}$, from 0.4 to 0.2 , and then increase back up to $\sim 0.3$ at 500 microns. The main result is that, on average, the dust-mass surface-density scale-length is about 1.8 times the stellar one derived from IRAC data and the $3.6 \mu \mathrm{m}$ surface brightness, and close to that in the UV. We found a mild dependence of the scale-lengths on the Hubble stage $T$ : the scale-lengths of the Herschel bands and the $3.6 \mu \mathrm{m}$ scale-length tend to increase from earlier to later types, the scale-length at $70 \mu \mathrm{m}$ tends to be smaller than that at longer sub-mm wavelength with ratios between longer sub-mm wavelengths and $70 \mu \mathrm{m}$ that decrease with increasing $T$. The scale-length ratio of SFR and stars shows a weak increasing trend towards later types. Our $\alpha_{\mathrm{CO}}$ determinations are in the range (0.3-9) $M_{\odot} \mathrm{pc}^{-2}\left(\mathrm{~K} \mathrm{~km} \mathrm{~s}^{-1}\right)^{-1}$, almost invariant by using a fixed dust-to-gas ratio mass (DGR) or a DGR depending on metallicity gradient.
\end{abstract}

Key words. dust, extinction - ISM: molecules - galaxies: ISM - galaxies: spiral - galaxies: structure - galaxies: photometry

\section{Introduction}

The mass of the interstellar medium (ISM) is composed of gas for $\approx 99 \%$ ( $\approx 75 \%$ as hydrogen and $\approx 25 \%$ as helium), and primarily of dust for the remaining $\approx 1 \%$. The ISM is of vital importance for the formation and evolution of galaxies since it is in the environment from which stars are formed. Stars indeed form inside dense molecular clouds, from the conversion of gas into stars (e.g., Krumholz 2011), while this gas is also ejected through stellar winds and supernova explosions (e.g.,

\footnotetext{
* DustPedia is a project funded by the EU under the heading "Exploitation of space science and exploration data". It has the primary goal of exploiting existing data in the Herschel Space Observatory and Planck Telescope databases.
}

Veilleux et al. 2005). The formation of hydrogen molecules $\left(\mathrm{H}_{2}\right)$, the raw material for star formation (SF), takes place on the surface of dust grains (Gould \& Salpeter 1963) that are formed from supernova/stellar ejecta and/or in the ISM (e.g., Draine 2003).

Although the dust constitutes a small percentage of the ISM, understanding its spatial distribution is of particular importance for two reasons. First, the dust affects our view of galaxies at different wavelengths, by absorbing ultraviolet (UV) and optical light and reemitting it in the infrared (IR). Correcting for dust extinction is usually the main source of uncertainty when deriving properties such as the star-formation rate (SFR), age, or metallicity (e.g., Calzetti et al. 1994; Buat \& Xu 1996; Calzetti 2001; Pérez-González et al. 2003). Second, the dust constitutes 
an important element in the chemical evolution of the ISM. Metals resulting from stellar nucleosynthesis are returned to the ISM, either as gas and solid grains condensed during the later stages of stellar evolution; they can later be destroyed and incorporated into new generations of stars. Elements are injected into the ISM at different rates (we refer to e.g., Dwek et al. 2009). For instance, atomic bounds of carbon are most likely responsible for the emission features, whose carriers are often identified with Polycyclic Aromatic Hydrocarbons (PAHs); they might originally have been produced in AGB stars, whose typical lifetimes are a few Gyr (e.g., Marigo et al. 2008; Cassarà et al. 2013; Villaume et al. 2015; Simonian \& Martini 2017). Other elements may be synthesized in more massive stars, which die as supernovae on shorter timescales. Thus, the abundance of the mid-IR (MIR) emission feature carriers and the dust-togas mass ratio (DGR) are expected to vary with the age of the stellar populations and correlate with the metal (e.g., oxygen and nitrogen) abundance of the gas (e.g., Galliano et al. 2008). However, dust formation results from a long chain of poorly-understood processes, from the formation and injection into the ISM of dust seeds formed in the atmospheres of AGB stars or in SNe ejecta, to grain growth and destruction in the ISM (e.g., Valiante et al. 2009; Asano et al. 2013; Mattsson et al. 2014; Rémy-Ruyer et al. 2014; Bocchio et al. 2016). Based on Draine (2009); only $~ 10 \%$ of interstellar dust is directly formed in stellar sources, with the remaining $\sim 90 \%$ being later condensed in the ISM. However, Jones \& Nuth (2011) found that the destruction efficiencies might have been severely overestimated. They concluded that the current estimates of global dust lifetimes could be uncertain by factors large enough to call into question their usefulness (we also refer to Ferrara et al. 2016).

The connection between dust, gas, and stars (and SF processes) has been investigated mainly through spatially unresolved studies of the ISM components and stars/SF (e.g., Buat et al. 1989; Buat 1992; Kennicutt 1989, 1998a; Deharveng et al. 1994; Cortese et al. 2012; Clark et al. 2015; Rémy-Ruyer et al. 2015; De Vis et al. 2017). Although these works are useful for characterizing global disk properties, intimately understanding the structure of galaxies requires resolved measurements. Thanks to IR and sub-millimeter (sub-mm) data from space telescopes (e.g., Spitzer and Herschel) together with ground-based facilities (e.g., IRAM-30 m, IRAM-PdBI now NOEMA, ALMA), we have also resolved measurements of dust and gas for large samples of nearby galaxies even beyond the Local Group (e.g., Wong \& Blitz 2002; Bigiel et al. 2008; Schruba et al. 2011; Muñoz-Mateos et al. 2009a; Casasola et al. 2015).

Although the ISM is distributed in an irregular manner between the stars, following a variety of structures (e.g., clouds, shells, holes, and filaments), the dust and molecular component of the ISM as well as stars are all generally distributed in a disk, often with an exponential decline of surface density with radius (e.g., Alton et al. 1998; Bianchi 2007; Bigiel et al. 2008; Muñoz-Mateos et al. 2009a; Hunt et al. 2015). The relative distribution of the dust components emitting at different IR and sub$\mathrm{mm}$ wavelengths provides information on the dominant heating mechanism of dust. In general, dust emission within galaxies is powered by radiation coming from both sites of recent SF and from more evolved stellar populations, as well as from the presence of active galactic nuclei (AGN, e.g., Draine \& Li 2007). There is a long-standing debate about the exact fraction of dust heating contributed by each stellar population (e.g., Law et al. 2011; Boquien et al. 2011; Bendo et al. 2012, 2015; Hunt et al. 2015), which depends on several factors such as the intrinsic spectral energy distribution (SED) of the stellar populations, the dust mass and optical properties, as well as the relative dust-star geometry.

Radial profiles of dust and stars are strong diagnostics to distinguish between the relative distributions and to understand how dust is heated. Based on this, Alton et al. (1998; see also Davies et al. 1999), by comparing the ISO $200 \mu \mathrm{m}$ observations (full width half maximum, $F W H M \simeq 2^{\prime}$ ) with improved resolution IRAS 60 and $100 \mu \mathrm{m}$ data $\left(F W H M \simeq 1-2^{\prime}\right)$ for eight nearby spiral galaxies, found that cold dust $(\sim 18-21 \mathrm{~K})$ detected by ISO becomes more dominant at larger radii with respect to warm dust $(\sim 30 \mathrm{~K})$ revealed by IRAS. The comparison of the $200 \mu \mathrm{m}$ images with $B$-band data also showed that the cold dust is radially more extensive than stars (the latter traced, in some measure, by the blue light). With higher resolution Spitzer observations of 57 galaxies in the SINGS sample, Muñoz-Mateos et al. (2009a) instead found that the distribution of the dust mass (derived from SED fits up to the longest available wavelength of $\left.160 \mu \mathrm{m} ; F W H M=38^{\prime \prime}\right)$ has a similar scale-length to that measured for stellar emission at $3.6 \mu \mathrm{m}$. However, the result is not incompatible with that of Alton et al. (1998), since near-infrared (NIR) surface-brightness profiles are steeper than in the optical.

The advent of Herschel allowed for the attainment of a competitive resolution beyond the peak of thermal dust emission. In a preliminary, qualitative analysis of the surface-brightness profiles of the two grand-design spirals NGC 4254 (M 99) and NGC 4321 (M 100), Pohlen et al. (2010) showed that cold dust detected with Herschel-SPIRE data $\left(250 \mu \mathrm{m}-F W H M=18^{\prime \prime}\right.$, $\left.350 \mu \mathrm{m}-F W H M=24^{\prime \prime}, 500 \mu \mathrm{m}-F W H M=36^{\prime \prime}\right)$ extends to at least the optical radius of the galaxy and shows features at similar locations as the stellar distribution. Scale-lengths at $250 \mu \mathrm{m}$ were derived for the 61 galaxies in the sample of the project Key Insights into Nearby Galaxies: Far-Infrared Survey with Herschel (KINGFISH, Kennicutt et al. 2011) by Hunt et al. (2015). They confirmed the results of Muñoz-Mateos et al. (2009a), that is, of the similarity between NIR and far-IR (FIR) scale-lengths. They did not provide, however, scale-lengths at the larger wavelength accessible to SPIRE in the sub-mm, nor for the dust-mass distribution that could be derived by resolved SED fitting. The scale-length of an averaged dust-mass profile was recently determined by Smith et al. (2016) from a stacking analysis of 45 low-inclination galaxies as part of the Herschel Reference Survey (HRS, Boselli et al. 2010). Since the main focus of the work was the detection of dust in the outskirts of spiral disks, scale-lengths for individual objects were not analyzed and shown.

In this paper, we study the spatial variations of dust, stars, gas, and SFR of a sample of 18 nearby, spiral, face-on galaxies extracted from the DustPedia sample ${ }^{1}$ by analyzing their surface-brightness and mass-surface-density profiles (we refer to Davies et al. 2017, for a detailed description of the DustPedia project and sample). To perform this we exploit the multi-wavelength (from UV to sub-mm wavelengths) DustPedia database $^{2}$. This paper covers - with continuity - a wider wavelength range, that is homogeneously treated with respect to previous works dedicated to scale-lengths of stars, dust and other ISM components in galaxies.

The paper is organized as follows. In Sect. 2 we outline the sample selection and in Sect. 3 we present the different data used in this work. In Sect. 4 we describe the adopted procedure to homogenize the dataset, and in Sect. 5 the methods adopted

\footnotetext{
http://www . dustpedia.com/

http://dustpedia.astro.noa.gr/
} 
to derive the mass of dust, stars, and gas and the SFR. Section 6 presents the surface-brightness profiles and mass and SFR surface-density profiles, their exponential fits and corresponding scale-lengths, while Sect. 7 describes the method we developed to solve for single $\mathrm{CO}$-to- $\mathrm{H}_{2}$ conversion factors by using dustand gas-mass profiles. Finally, in Sect. 8 we summarize our main results.

\section{The sample selection}

For the purpose of this work, we selected large spiral galaxies from the DustPedia sample with a small (or moderate) disk inclination, imaged over their whole extent with both PACS and SPIRE in Herschel. We first searched for objects with $D_{25}>6^{\prime 3}$ in the $B$-band and with Hubble stage $T$ ranging from 1 to 8 (including galaxies with classifications from $\mathrm{Sa}$ to $\mathrm{Sdm}$, as listed in the Nearby Galaxy Atlas; Tully 1988). We then refined the search after measuring the extent of the galaxy in the $250 \mu \mathrm{m}$ images at the isophote of $12 \mathrm{mJy} / \mathrm{beam}$ (i.e., at twice the confusion noise, a sensitivity achieved in all SPIRE images; Nguyen et al. 2010). A total of 18 galaxies were found with PACS and SPIRE data and sub-mm minor-to-major axis ratio $(d / D)_{\text {sub-mm }} \geq 0.4$ and $D_{\text {sub-mm }} \geq 9^{\prime}$, corresponding to $\approx 15$ resolution elements in the SPIRE $500 \mu \mathrm{m}$ maps.

The final sample includes galaxies with all sorts of peculiarities, such as the presence of bars and/or signatures of interaction with companions. All galaxies are indeed present in the sample of interacting galaxies of Casasola et al. (2004) that includes more than 1000 galaxies appearing to be clearly interacting with nearby objects and presenting tidal tails or bridges, merging systems, and galaxies with disturbed structures. We also checked for the presence of AGN in our sample. Ten of the eighteen sample galaxies are classified as low-luminosity AGN $\left(L_{\mathrm{X}}<10^{42} \mathrm{erg} \mathrm{s}^{-1}\right)$, including Seyferts and low-ionization nuclear emission-line region (LINER) galaxies, while the reaming sources have H II nuclei (except for NGC 300 for which there is no nuclear classification, to our knowledge). Table 1 collects the main properties of the sample galaxies, that are listed in order of decreasing $D_{25}$.

\section{The data}

In this section we present the data collected for the studied galaxy sample, whose main properties are listed in Tables 2 and 3 . Most of the data used have been retrieved from the DustPedia database except for molecular $\left({ }^{12} \mathrm{CO}\right)$ and atomic (HI) gas maps, a fraction of the optical images, and information on metallicity.

\subsection{Herschel data and their reduction}

We used both PACS (70, 100, and $160 \mu \mathrm{m})$ and SPIRE (250, 350 , and $500 \mu \mathrm{m})$ Herschel maps. PACS images have FWHM of 6,8 , and $12^{\prime \prime}$ at 70,100 , and $160 \mu \mathrm{m}$, respectively, and SPIRE images of 18,24 , and $36^{\prime \prime}$ at 250,350 , and $500 \mu \mathrm{m}$, respectively. The reduction of these data for the entire DustPedia sample, including galaxies studied in this paper, has been performed by the DustPedia team. Davies et al. (2017) describe in detail the data reduction adopted for both PACS and SPIRE observations. Final reduced Herschel images as well as the other ones present in the DustPedia database are in units of $\mathrm{Jy} \mathrm{pix}^{-1}$ (for details on

\footnotetext{
$3 D_{25}$ is defined as the length the projected major axis of a galaxy at the isophotal level $25 \mathrm{mag} / \mathrm{arcsec}^{2}$ in the $B$-band (this is the diameter of the galaxy if it is a disk).
}

the database, we refer to Clark et al. 2017). The Herschel images have been used to derive the FIR/sub-mm light distribution and the dust-mass surface-density profile (Sect. 5.1).

\subsection{GALEX data}

We used ultraviolet (UV) images of our sample galaxies observed by the Galaxy Evolution Explorer (GALEX) satellite in its far-UV (FUV, $\lambda_{\text {eff }}=1516 \AA$ ) and near-UV (NUV, $\lambda_{\text {eff }}=2267 \AA$ ) bands. The GALEX images have FWHM in the range 4".0-4".5 and $5 " .0-5 " .5$ for the FUV and NUV bands, respectively.

Massive, young stars emit most of their energy in this part of the spectrum and, at least in star-forming galaxies, they outshine the emission from any other stage of the evolution of a composite stellar population (e.g., Bruzual \& Charlot 2003). Therefore, the flux emitted in the UV in spiral (and irregular) galaxies is an excellent measure of the current SFR (e.g., Kennicutt 1998b). Additionally, the light emitted in the UV can be very efficiently absorbed by dust and then re-emitted at FIR wavelengths. Therefore, an analysis of the energy budget comparing the IR and UV emission is a powerful tool to determine the dust attenuation of light at all wavelengths (e.g., Buat et al. 2005; Cortese et al. 2006; Mao et al. 2012). However, an analysis on the energy budget is beyond the scope of this paper. The GALEX images have been used to derive the UV stellar light distribution and the SFR surface-density profiles together with IR emission (Sect. 5.4).

\subsection{SDSS and SINGS data}

We used two optical datasets. The first one consists of images originally from the Sloan Digital Sky Survey (SDSS), Data Release 12 (DR12 ${ }^{4}$, York et al. 2000). We used images in $g$ $(4686 \AA)$ and $i$ (7480 $⿱$ ) $)$ wavebands for nine sample galaxies. The FWHM is of $\sim 1$ "'4 and $\sim 1$ '!2 for $g$ and $i$ waveband, respectively. The second optical dataset we used is not contained in the DustPedia database. These images come from "The Spitzer Infrared Nearby Galaxies Survey - Physics of the Star-Forming ISM and Galaxy Evolution" (SINGS ${ }^{5}$ ) collaboration, that obtained optical BVRI data for the galaxies in the SINGS sample. We used $B(4360 \AA)$ and $R(7480 \AA)$ wavebands for other two sample galaxies. The FWHM is of $\sim 1-2^{\prime \prime}$ in both wavebands.

The optical light revealed in $g$ and $i$ SDSS and $B$ and $R$ SINGS images traces the young blue stellar content of a galaxy (e.g., Bruzual \& Charlot 1993; Maraston 2005; Piovan et al. 2006; Cassarà et al. 2013). These images have been used both to derive the optical stellar light and stellar-mass surface-density profiles of the sample galaxies (Sect. 5.2).

\subsection{Spitzer data}

We used NIR images, originally obtained with the IRAC camera on Spitzer at 3.6 and $4.5 \mu \mathrm{m}$. These images have a median FWHM of $\sim 1$ '! 7 for both wavebands.

The IRAC 3.6 and $4.5 \mu \mathrm{m}$ bands are sensitive mainly to the dust penetrated old stellar component. Since these NIR channels are nearly minimally sensitive to dust absorption and emission (e.g., Fazio et al. 2004; Willner et al. 2004), the corresponding

\footnotetext{
4 DR12 is the final data release of the SDSS-III, containing all SDSS observations through July 2014 and encompassing more than one-third of the entire celestial sphere, https: //WwW. sdss3.org/

5 http://irsa.ipac.caltech.edu/data/SPITZER/SINGS/, see also Dale et al. (2017) for the new recalibration of the whole dataset.
} 
A\&A 605, A18 (2017)

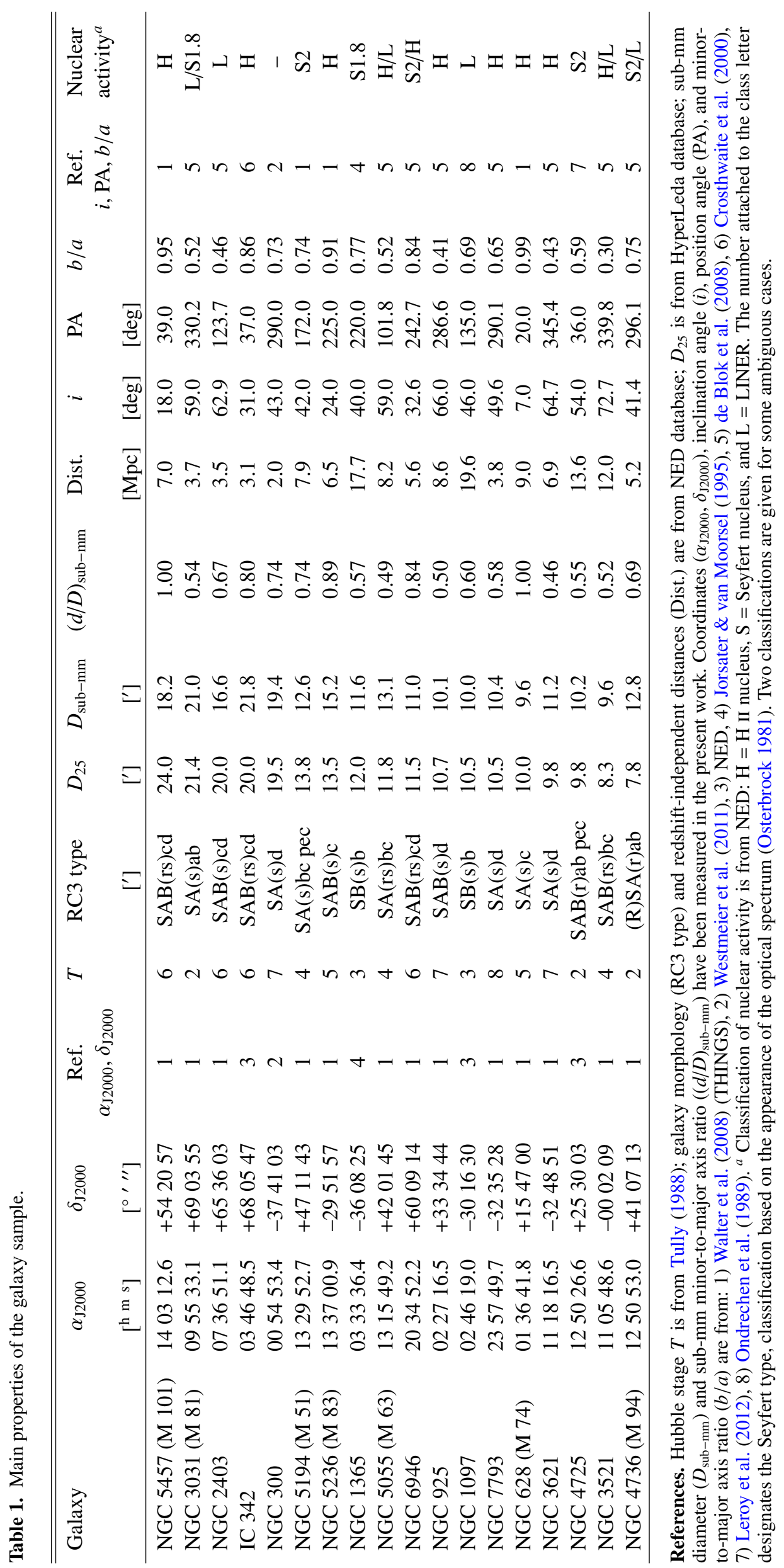


Table 2. Main properties of the Herschel images and the ancillary ones used for this work.

\begin{tabular}{|c|c|c|c|c|}
\hline Instrument/survey & Wavelength & $\begin{array}{c}F W H M \\
{\left[{ }^{\prime \prime}\right]}\end{array}$ & Ref. $F W H M$ & $\begin{array}{c}\text { Pixel size } \\
{\left[{ }^{\prime \prime}\right]}\end{array}$ \\
\hline$G A L E X-\mathrm{FUV}^{a}$ & $1516 \AA$ & $4.0-4.5$ & 1 & 3.2 \\
\hline$G A L E X-\mathrm{NUV}^{a}$ & $2267 \AA$ & $5.0-5.5$ & 1 & 3.2 \\
\hline SDSS $-g^{b}$ & $4686 \AA$ & 1.4 & 2 & 0.45 \\
\hline SDSS $-i^{b}$ & $7480 \AA$ & 1.2 & 2 & 0.45 \\
\hline SINGS- $B^{c}$ & $4360 \AA$ & $\sim 1-2$ & 3 & 0.433 \\
\hline SINGS- $R^{c}$ & $6440 \AA$ & $\sim 1-2$ & 3 & 0.433 \\
\hline Spitzer-IRAC- $1^{a}$ & $3.6 \mu \mathrm{m}$ & 1.7 & 4 & 0.75 \\
\hline Spitzer-IRAC-2 ${ }^{a}$ & $4.5 \mu \mathrm{m}$ & 1.7 & 4 & 0.75 \\
\hline WISE-W3 ${ }^{a}$ & $12 \mu \mathrm{m}$ & 6.8 & 5 & 1.375 \\
\hline WISE-W4 ${ }^{a}$ & $22 \mu \mathrm{m}$ & 11.8 & 5 & 1.375 \\
\hline Herschel-PACS ${ }^{d}$ & $70 \mu \mathrm{m}$ & 6 & 6 & 2 \\
\hline Herschel-PACS ${ }^{e}$ & $100 \mu \mathrm{m}$ & 8 & 6 & 3 \\
\hline Herschel-PACS ${ }^{a}$ & $160 \mu \mathrm{m}$ & 12 & 6 & 4 \\
\hline Herschel-SPIRE ${ }^{a}$ & $250 \mu \mathrm{m}$ & 18 & 7 & 6 \\
\hline Herschel-SPIRE ${ }^{a}$ & $350 \mu \mathrm{m}$ & 24 & 7 & 8 \\
\hline Herschel-SPIRE ${ }^{a}$ & $500 \mu \mathrm{m}$ & 36 & 7 & 12 \\
\hline
\end{tabular}

Notes. These images are from the DustPedia database, except for the SINGS ones. ${ }^{(a)}$ Available data for the whole sample. ${ }^{(b)}$ Available data for nine sample galaxies: NGC 5457 (M 101), NGC 2403, NGC 3031 (M 81), NGC 5194 (M 51), NGC 5055 (M 63), NGC 4736 (M 94), NGC 3521, NGC 4725, and NGC 628 (M 74). ${ }^{(c)}$ Data for NGC 3621 and NGC 1097; for these two galaxies SDSS- $g$ and SDSS- $i$ data are not available. ${ }^{(d)}$ Available data for the whole sample except for NGC 300. ${ }^{(e)}$ Available data for the whole sample except for NGC 2403, NGC 3031 (M 81), NGC 5194 (M 51), and NGC 5236 (M 83). The numbers in Col. (4) refer to the following references: 1) Morrissey et al. (2007); 2) Bramich \& Freudling (2012); 3) http://irsa.ipac.caltech.edu/data/SPITZER/SINGS/; 4) see "IRAC Instrument Handbook", http://irsa.ipac. caltech. edu/data/SPITZER/docs/irac/iracinstrumenthandbook/; 5) see "Explanatory Supplement to the WISE All-Sky Data Release Products", http://wise2.ipac.caltech.edu/docs/release/allsky/expsup/index.html; 6) see "PACS Observer's Manual", http://herschel.esac.esa.int/Docs/PACS/html/pacs_om.html; 7) see "The Spectral and Photometric Imaging Receiver (SPIRE) Handbook", http://herschel . esac.esa.int/Docs/SPIRE/html/spire_om.html.

images are used as tracers of the stellar mass distribution (e.g., Kennicutt et al. 2003; Helou et al. 2004). Younger (hotter) stars are not expected to contribute significantly to the observed stellar emission. However, due to their strong UV fluxes, these younger stars can heat their surrounding dust, which, in turn, re-radiates at longer wavelengths and can also account for a significant fraction of the light at $3.6 \mu \mathrm{m}$ (e.g., Meidt et al. 2012). The IRAC 3.6 and $4.5 \mu \mathrm{m}$ images have been used both to derive the NIR stellar light and stellar-mass surface-density profiles of the sample galaxies (see Sect. 5.2).

\subsection{WISE data}

We used MIR images at $12 \mu \mathrm{m}$ and $22 \mu \mathrm{m}$ bands originally taken with the Wide-field Infrared Survey Explorer (WISE, Wright et al. 2010). The median FWHM is 6".8 and 11'.8 for the $12 \mu \mathrm{m}$ and $22 \mu \mathrm{m}$ images, respectively.

The W3 band covers the complex of emission bands at 8$13 \mu \mathrm{m}$ imputed to carbonaceous material as well as the broad silicate absorption feature at $10 \mu \mathrm{m}$. The $\mathrm{W} 4$ band, on the other hand, is free of these emission features: it therefore serves as a good measure of the pure dust continuum, sampling warm dust in the high temperature tail of the typical distribution of grains and/or non-thermal emission from stochastically heated grains. The W3 and W4 WISE images have been used to derive the corresponding MIR emission profiles and the $22 \mu \mathrm{m}-\mathrm{W} 4 \mathrm{im}-$ ages have been combined with the GALEX-FUV ones to correct the FUV luminosity for dust attenuation, and subsequently, derive maps tracing the current SFR surface-density profiles (see Sect. 5.4).

\section{6. $\mathrm{HI}$ and ${ }^{12} \mathrm{CO}$ images}

We derived gas-mass profiles for the galaxies in our sample by using images of the HI $21 \mathrm{~cm}$ emission line and of different ${ }^{12} \mathrm{CO}$ emission lines for tracing the atomic and molecular gas component, respectively. The data were available through various surveys, catalogs, and individual projects. For 15 galaxies we retrieved HI data, for 13 galaxies ${ }^{12} \mathrm{CO}$ data, and for 12 of these galaxies we have data for both $\mathrm{HI}$ and ${ }^{12} \mathrm{CO}$ emission lines. In Table 3 we list the collected $\mathrm{HI}$ and ${ }^{12} \mathrm{CO}$ images we used. These images are already reduced and details on the data reduction procedures are contained in the case-by-case references quoted in Table 3. In the following, we give the main information on these images.

Concerning the atomic gas component, for 13 galaxies, HI maps are from "The HI Nearby Galaxy Survey" (THINGS, Walter et al. 2008) ${ }^{6}$. THINGS is a program undertaken at the NRAO Very Large Array (VLA) to perform $21 \mathrm{~cm} \mathrm{HI} \mathrm{observa-}$ tions of 34 nearby galaxies at an angular resolution of 6 ". From the THINGS data repository we extracted reduced integrated HI intensity maps ("moment 0 ") in units of Jy beam ${ }^{-1} \mathrm{~m} \mathrm{~s}^{-1}$. For IC 342 we used the $21 \mathrm{~cm}$ HI map available from the NED catalog in units of Jy beam ${ }^{-1} \mathrm{~m} \mathrm{~s}^{-1}$ and obtained with the NRAO VLA at the angular resolution of $38^{\prime \prime}$. For NGC 4725, the $21 \mathrm{~cm}$

6 http://www . mpia.de/THINGS/Overview .html 
Table 3. Collected HI $21 \mathrm{~cm}$ and ${ }^{12} \mathrm{CO}(1-0$ or 2-1) emission line data used to derive the mass surface density of the atomic and molecular gas, respectively.

\begin{tabular}{|c|c|c|c|c|c|c|}
\hline Galaxy & Instrument $\mathrm{HI}(F W H M)$ & Ref. HI & Pixel size HI & Instrument ${ }^{12} \mathrm{CO}(F W H M)$ & Ref. ${ }^{12} \mathrm{CO}\left({ }^{12} \mathrm{CO}\right.$ line $)$ & Pixel size ${ }^{12} \mathrm{CO}$ \\
\hline NGC 5457 (M 101) & $\operatorname{VLA}\left(6^{\prime \prime}\right)$ & 1 & $11^{\prime \prime}$ & IRAM $30 \mathrm{~m}\left(11^{\prime \prime}\right)$ & $4(2-1)$ & $2^{\prime \prime}$ \\
\hline NGC 3031 (M 81) & $\operatorname{VLA}\left(6^{\prime \prime}\right)$ & 1 & $11^{\prime \prime}$ & BIMA $\left(6^{\prime \prime}\right)$ & $5(1-0)$ & $1^{\prime \prime}$ \\
\hline NGC 2403 & VLA $\left(6^{\prime \prime}\right)$ & 1 & $11^{\prime \prime}$ & IRAM $30 \mathrm{~m}\left(11^{\prime \prime}\right)$ & $4(2-1)$ & $2^{\prime \prime}$ \\
\hline IC 342 & $\operatorname{VLA}\left(38^{\prime \prime}\right)$ & 2 & $6^{\prime \prime}$ & NRO $45 \mathrm{~m}\left(15^{\prime \prime}\right)$ & $6(1-0)$ & $1^{\prime \prime}$ \\
\hline \multicolumn{7}{|l|}{ NGC 300} \\
\hline NGC 5194 (M 51) & $\operatorname{VLA}\left(6^{\prime \prime}\right)$ & 1 & $11^{\prime \prime}$ & IRAM $30 \mathrm{~m}\left(11^{\prime \prime}\right)$ & $4(2-1)$ & $2^{\prime \prime}$ \\
\hline NGC 5236 (M 83) & $\operatorname{VLA}\left(6^{\prime \prime}\right)$ & 1 & $11^{\prime \prime}$ & & & \\
\hline \multicolumn{7}{|l|}{ NGC 1365} \\
\hline NGC 5055 (M 63) & $\operatorname{VLA}\left(6^{\prime \prime}\right)$ & 1 & $11^{\prime \prime}$ & IRAM $30 \mathrm{~m}\left(11^{\prime \prime}\right)$ & $4(2-1)$ & $2^{\prime \prime}$ \\
\hline NGC 6946 & $\operatorname{VLA}\left(6^{\prime \prime}\right)$ & 1 & $11^{\prime \prime}$ & IRAM $30 \mathrm{~m}\left(11^{\prime \prime}\right)$ & $4(2-1)$ & $2^{\prime \prime}$ \\
\hline NGC 925 & $\operatorname{VLA}\left(6^{\prime \prime}\right)$ & 1 & $11^{\prime \prime}$ & IRAM $30 \mathrm{~m}\left(11^{\prime \prime}\right)$ & $4(2-1)$ & $2^{\prime \prime}$ \\
\hline NGC 1097 & & & & ATFN Mopra 22 m (30") & $7(1-0)$ & $1^{\prime \prime}$ \\
\hline NGC 7793 & VLA $\left(6^{\prime \prime}\right)$ & 1 & $11^{\prime \prime}$ & & & \\
\hline NGC 628 (M 74) & $\operatorname{VLA}\left(6^{\prime \prime}\right)$ & 1 & $11^{\prime \prime}$ & IRAM 30 m (11") & $4(2-1)$ & $2^{\prime \prime}$ \\
\hline NGC 3621 & VLA $\left(6^{\prime \prime}\right)$ & 1 & $11^{\prime \prime}$ & & & \\
\hline NGC 4725 & WSRT (13"'22) & 3 & $13 " \prime 22$ & IRAM 30 m (11") & $4(2-1)$ & $2^{\prime \prime}$ \\
\hline NGC 3521 & $\operatorname{VLA}\left(6^{\prime \prime}\right)$ & 1 & $11^{\prime \prime}$ & IRAM $30 \mathrm{~m}\left(11^{\prime \prime}\right)$ & $4(2-1)$ & $2^{\prime \prime}$ \\
\hline NGC 4736 (M 94) & $\operatorname{VLA}\left(6^{\prime \prime}\right)$ & 1 & $11^{\prime \prime}$ & IRAM $30 \mathrm{~m}\left(11^{\prime \prime}\right)$ & $4(2-1)$ & $2^{\prime \prime}$ \\
\hline
\end{tabular}

References. For the HI and ${ }^{12}$ CO FWHM values are: 1) THINGS survey (Walter et al. 2008); 2) NED (Crosthwaite et al. 2001); 3) WHISP survey from NED (Swaters et al. 2002); 4) HERACLES survey (Leroy et al. 2012); 5) BIMA SONG survey (Helfer et al. 2003); 6) Nobeyama CO Atlas of Nearby Spiral Galaxies (Kuno et al. 2007); 7) priv. comm. (PI's proposal: M. W. L. Smith).

HI map is from "Westerbork observations of neutral Hydrogen in Irregular and SPiral galaxies" (WHISP, Swaters et al. 2002) ${ }^{7}$, a survey undertaken at the Westerbork Synthesis Radio Telescope (WSRT) at a resolution of $13^{\prime \prime} .22$. We downloaded the reduced integrated HI intensity map from NED in Westerbork Units $\left(1 \mathrm{~W} . \mathrm{U} .=2.2 \times 10^{20} \mathrm{H}\right.$ atoms $\mathrm{cm}^{-2}$ ).

Concerning the molecular gas component, for ten sample galaxies we used ${ }^{12} \mathrm{CO}(2-1)$ line intensity $(230 \mathrm{GHz} / 1.3 \mathrm{~mm}$, rest frame) maps from "The HERA CO-Line Extragalactic Survey" (HERACLES, Leroy et al. 2009) ${ }^{8}$. HERACLES is a Large Program that used the IRAM $30 \mathrm{~m}$ telescope to map the ${ }^{12} \mathrm{CO}(2-1)$ emission from 48 nearby galaxies at the angular resolution of $11^{\prime \prime}$. From HERACLES data repository we extracted the reduced integrated intensity maps and associated uncertainty, in units of $\mathrm{K} \mathrm{km} \mathrm{s}^{-1}$. For NGC 3031 (M 81) we used the ${ }^{12} \mathrm{CO}(1-0)$ line intensity $(115 \mathrm{GHz} / 2.6 \mathrm{~mm}$, rest frame) map from BIMA SONG and available from NED in units of Jy beam ${ }^{-1} \mathrm{~m} \mathrm{~s}^{-1}$. BIMA SONG is a systematic imaging study of the ${ }^{12} \mathrm{CO}(1-0)$ molecular line emission within the centers and disks of 44 nearby spiral galaxies performed with the 10-element Berkeley-Illinois-Maryland Association (BIMA) millimeter interferometer at an angular resolution of $6^{\prime \prime}$. For IC 342 we downloaded the ${ }^{12} \mathrm{CO}(1-0)$ line intensity map from "Nobeyama $\mathrm{CO}$ Atlas of Nearby Spiral Galaxies" (Kuno et al. 2007) ${ }^{9}$ in units of $\mathrm{K} \mathrm{km} \mathrm{s}^{-1}$, a ${ }^{12} \mathrm{CO}(1-0)$ survey of 40 nearby spiral galaxies performed with the NRO $45 \mathrm{~m}$ telescope at an angular resolution of $15^{\prime \prime}$. For NGC 1097 , we used the ${ }^{12} \mathrm{CO}(1-0)$ line intensity map in units of $\mathrm{K} \mathrm{km} \mathrm{s}^{-1}$ observed by us with the ATFN Mopra $22 \mathrm{~m}$ telescope (PI: M. W. L. Smith) at an angular resolution of $30^{\prime \prime}$.

\subsection{Metallicity abundances}

We also searched for metallicity abundance information for the face-on DustPedia sample. Pilyugin et al. (2014) compiled [O II] $\lambda 3727+\lambda 3729,[\mathrm{O}$ III] $\lambda 5007,[\mathrm{~N} \mathrm{II}] \lambda 6584$, and [S II] $\lambda 6717+\lambda 6731$ emission lines (all normalised to $\mathrm{H} \beta$ ) for

\footnotetext{
7 http://www .astro.rug.nl/ whisp/

8 http://www.mpia.de/HERACLES/Data.html

9 http://www.nro.nao.ac.jp/ nro45mrt/html/Coatlas/
}

around 3740 published spectra of $\mathrm{H}$ II regions across the optical disks of 130 nearby late-type galaxies. Using these emission lines they determined homogeneous oxygen $(\mathrm{O} / \mathrm{H})$ and nitrogen $(\mathrm{N} / \mathrm{H})$ abundance distributions and fitted radial profiles within the isophotal radius in every galaxy following this equation:

$12+\log (\mathrm{O} / \mathrm{H})=12+\log (\mathrm{O} / \mathrm{H})_{r_{0}}+C_{\mathrm{O} / \mathrm{H}} \times\left(r / r_{25}\right)$,

where $12+\log (\mathrm{O} / \mathrm{H})_{r_{0}}$ is the oxygen abundance at $r_{0}=0$, that is, the extrapolated central oxygen abundance, $C_{\mathrm{O} / \mathrm{H}}$ is the slope of the oxygen abundance gradient expressed in terms of dex $r_{25}{ }^{-1}$, and $r / r_{25}$ is the fractional radius (the galactocentric distance normalized to the disk's isophotal radius $r_{25}$ ). All galaxies of our sample are contained in Pilyugin et al. (2014) and we used their metallicity values (see their Table 2) to study the variation of the $\mathrm{CO}$-to- $\mathrm{H}_{2}$ conversion factor as a function of $\mathrm{O} / \mathrm{H}$ abundance (see Sects. 5.3 and 7).

We stress that different strong-line diagnostics and calibration methodologies (e.g., photoionization models versus empirical electronic temperature, $T_{\mathrm{e}}$, derivations) yield substantial systematic offsets in the inferred gas metallicities (e.g., Kennicutt et al. 2003; Moustakas et al. 2010; López-Sánchez et al. 2012; Bresolin et al. 2016), reaching values up to 0.7 dex (Kewley \& Ellison 2008). Methods calibrated from $T_{\mathrm{e}}$ measurements tend to occupy the bottom of the abundance scale. Since, to our knowledge, abundances derived with the $T_{\mathrm{e}}$-method are not available for the whole sample, we take advantage of using metallicity values derived from the same calibration for our entire sample to have an homogeneous treatment of data.

\section{Image treatment}

As described in Clark et al. (2017), UV, optical, and NIR images in the DustPedia database have Galactic foreground stars removed. The same procedure was used to remove foreground stars in the additional SINGS images used in this work. Sky subtraction was instead performed by defining a few (at least five) rectangular regions of blank sky far away from everything, containing no detectable stars or other features. The average brightness level of these regions has therefore been subtracted from the 
image. While the choice of the correct sky value might affect the derivation of surface-brightness profiles and their scale-lengths, we checked that it has little influence for the inner disk profiles we analyze here. Even for the outer parts of the disk in the 70 and $100 \mu \mathrm{m}$ defining the extent of our analysis (see Sect. 6.1), changes in the adopted sky values, within the uncertainty of their estimate, produce negligible variations in the results.

All images from GALEX-FUV at $1516 \AA$ to HerschelSPIRE at $350 \mu \mathrm{m}$ (Table 2) and HI and ${ }^{12} \mathrm{CO}$ maps (Table 3) were convolved to the resolution of the $500 \mu \mathrm{m}$ (Herschel-SPIRE) maps $\left(36^{\prime \prime}\right)$. We chose the resolution of $500 \mu \mathrm{m}$, which is used in our SED fitting procedures and defines the resolution of the derived dust maps (Sect. 5.1). For most cases, convolution kernels to the $500 \mu \mathrm{m}$ PSF are available in Aniano et al. (2011). The higher-resolution gas maps, instead, were convolved to the lower resolution using simple Gaussian kernels. The HI map of IC 342 was left unaltered, since its angular resolution is close to that of $500 \mu \mathrm{m}$.

Then, all maps have been resampled to the $500 \mu \mathrm{m}$ map pixel size (12", see Table 2). These procedures were performed using IDL $^{10}$, IRAF $^{11}$, and IRAM/GILDAS ${ }^{12}$ (Guilloteau \& Lucas 2000) software packages.

\section{Surface density of galaxy properties}

In this section we describe the adopted techniques to derive the mass surface density of dust, stars, and gas and the SFR surface density.

\subsection{Dust mass}

The dust mass surface density has been derived by comparing a modelled SED, convolved with the Herschel filter response functions, with the observed data at each position within a galaxy. The parameters of the model were retrieved using a standard $\chi^{2}$ minimization technique.

We adopted the optical properties and grain size distributions of the THEMIS ${ }^{13}$ dust model, as described in Jones et al. (2013) and successive updates (Köhler et al. 2014; Ysard et al. 2015; Jones et al. 2017). We assumed that the emission is due to grains exposed to a radiation field with the same spectrum as that of the local interstellar radiation field (LISRF, Mathis et al. 1983), and intensity scaled up and down via the parameter $U$ to account for different heating environments within the galaxy ( $U=1$ for the same conditions as in the Solar neighborhood). The dust emission for the various $U$ values, including both thermal and stochastic processes, was computed with the DustEM software (Compiègne et al. 2011).

Our approach is a simplified version of the more complex Draine \& Li (2007) procedure, which includes both a radiation field of intensity $U=U_{\min }$, responsible for most of the thermal peak at $\lambda \geq 100 \mu \mathrm{m}$, and a power-law distribution of higher intensity radiation fields $\left(U>U_{\min }\right)$. This second component, though necessary to describe the emission at shorter

\footnotetext{
10 http: //idlastro.gsfc.nasa.gov/, Landsman (1993).

11 IRAF is the Image Reduction and Analysis Facility. IRAF is written and supported by the National Optical Astronomy Observatories (NOAO) in Tucson, Arizona. NOAO is operated by the Association of Universities for Research in Astronomy (AURA), Inc. under cooperative agreement with the National Science Foundation.

12 http: //WWW . iram. fr/IRAMFR/GILDAS/

13 The Heterogeneous Evolution Model for Interstellar Solids, http://www.ias.u-psud. fr/themis/index.html
}

wavelengths, contributes little to the dust mass determination. In fact, applications of the method to both global and resolved SEDs (e.g. Dale et al. 2012; Aniano et al. 2012, respectively) show that only a minor fraction of the dust mass (smaller than a few percent) is exposed to the more intense fields. Thus, fits to $\lambda \geq 100 \mu \mathrm{m}$ Herschel SEDs assuming that dust is heated by a single radiation field with $U=U_{\min }$ can retrieve the bulk of the dust mass (see also Magrini et al. 2011; Bianchi 2013).

Initially, we derived the dust mass surface density $\Sigma_{\text {dust }}$ and radiation field intensity $U$ for all the pixels where the Herschel surface brightness was larger than $2 \sigma$ in all bands with $\lambda \geq$ $160 \mu \mathrm{m}$. We thus produced $\Sigma_{\text {dust }}$ and $U$ maps, in a way analogous to Magrini et al. (2011). However, this procedure could have biased the results to flatter gradients; about half of the galaxy fits in the outer parts of the objects were possible only for a fraction of the pixels, and only those were counted in the profile determination. Conversely, by assuming that the non-fitted pixels had null values for $\Sigma_{\text {dust }}$ and $U$, the results would have been biased to steeper gradients. Thus, we resorted to using the method of Hunt et al. (2015): the dust-mass surface-density profiles are directly derived by fitting, for each radial bin, the SED resulting from the azimuthally averaged surface-brightness profiles. The method was not our first choice, because of other potential biases induced by the possible temperature mixing along the isophotal ellipses. However, for the regions where there is a full pixel coverage of the azimuthal bins, there is little differences with our initial, pixel-by-pixel, determinations; instead, for the external part of a galaxy, the Hunt et al. (2015) procedure allows us to extend the profiles to larger distances from the center. Errors on the fit results were estimated using a Monte Carlo bootstrapping technique, assuming as errors for the surface-brightness profile the sum, in quadrature, of the standard deviation of the mean along the elliptical annulus, and the calibration error of each band. The profile determination is further described in Sect. 6.1.

Besides the bands with $\lambda \geq 160 \mu \mathrm{m}$, the $100 \mu \mathrm{m}$ data, when available, were included in the fit (or used as an upper limit in pixels with $S / N<2$ ). For galaxies with available $70 \mu \mathrm{m}$ images, the data were used as an upper limit, in order to avoid contamination from grains heated by more intense radiation fields that we do not include in our approach. The inclusion of upper limits in the fit was achieved as in Rémy-Ruyer et al. (2013): first, a fit was performed without $70 \mu \mathrm{m}$ data or $<2 \sigma$ signal at $100 \mu \mathrm{m}$; if the fitted SED exceeded the flux at $70 \mu \mathrm{m}$ or $2 \sigma$ at $100 \mu \mathrm{m}$, the fit was repeated including those datapoints. Typical average parameters retrieved from the fits are, at $r=0.5 \times r_{25}, U=1-4$ and $\Sigma_{\text {dust }}=0.02-0.1 M_{\odot} \mathrm{pc}^{-2}$, depending on the galaxy. An example of the fits is given in Fig. 1.

Given the various reports on the ability of different kinds of fits to retrieve the correct dust masses (and thus the dust surface mass densities), we also experimented with other approaches: a single-temperature modified-blackbody (MBB) fit with the averaged absorption cross-section of our reference dust model; a MBB with a power-law absorption cross section with spectral index $\beta=1.5$ and 2, scaled on the Milky Way emissivity (Bianchi 2013); or emission spectra from the Draine \& Li (2007) and Compiègne et al. (2011) dust models. We also tried to estimate the effect of a (maximum) contribution from hot dust by fitting a MBB to all galaxies with available $70 \mu \mathrm{m}$ data after assuming that the entire $70 \mu \mathrm{m}$ flux was due to dust at 40 or $50 \mathrm{~K}$ (and correcting the longer wavelength bands accordingly). The dust-mass surface density changes from fit to fit, and, in particular, when different dust models are used: values obtained with the Draine \& Li (2007) emission templates are about 2.7 times larger than those from our reference THEMIS model, and those 


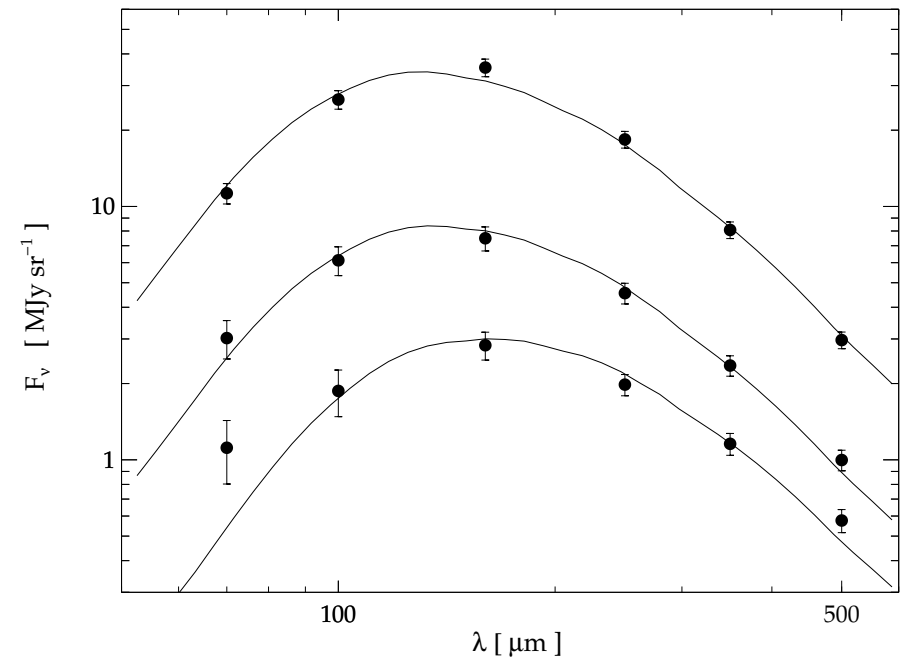

Fig. 1. Example SED fits: the datapoints are from three different positions on NGC 5457 (M 101) at $R \approx 0.3,0.5$, and $0.7 \times r_{25}$, corresponding to the local average heating conditions (fitted $U \approx 3,2$ and 1 , respectively, from top to bottom).

with the Milky Way-scaled power law emissivities of Bianchi (2013) a factor 1.5 times higher. The difference is due almost entirely to the difference in the optical properties of the dust models and it does not imply in itself that a particular model is better or worse than the others. For example, in Bianchi (2013) the dust masses of galaxies in the KINGFISH sample were derived assuming that the global Herschel SED could be described by a single temperature modified blackbody and with a powerlaw absorption cross-section fitting the average properties of the dust model used in the Draine \& Li (2007) emission templates, that of Draine (2003). We repeated the same estimate by using the absorption cross-section $k_{\lambda}=8.06 \times(250 \mu \mathrm{m} / \lambda)^{1.85} \mathrm{~cm}^{2} / \mathrm{g}$, a fit to the average properties of the THEMIS model between 70 and $700 \mu \mathrm{m}$. The ratio of the dust masses derived with the two models is 2.7 , essentially the same found in this work, despite the fact that the analysis is done here for a different sample, for specific positions within each galaxy and without assuming a single temperature. The difference is due mostly to the larger average absorption cross-section of the THEMIS model with respect to Draine (2003), a factor 2 at $250 \mu \mathrm{m}$ which converts to a factor 2 larger dust masses for Draine (2003); and in part to the steeper spectral index of Draine (2003) $(\beta=2.08$ vs. 1.78 in THEMIS) which results, for MBB fits, in smaller temperatures and thus in a further increase in the dust mass estimate to reproduce the same SED. Nevertheless, we found that the profiles (i.e., the gradients) have small differences, with changes in the fitted scale-length that can be, at most, as large as the error estimate of the fitting procedure (Sect. 6.1 and Table 6) but are in most cases smaller.

A decreasing spectral index for the absorption cross-section with radius, and thus possible variations in the dust properties along the disk, has been reported for MBB fits to M 31 (Smith et al. 2012a) and M 33 (Tabatabaei et al. 2014). Though this change in properties might affect the dust gradients, we did not try fits with varying $\beta$, as the dust-mass determination would also need a knowledge of the unknown variations of the absorption cross-section normalization with the environment. We only note that an apparent, flatter, absorption cross-section for colder dust at larger radii can be produced by the mixing due to the different temperatures attained by grains of different size and materials (Hunt et al. 2015). This behavior, if important in the determination of the dust gradients, should be captured by our fitting procedure, that uses the full THEMIS grain distribution.

Since the aim of this paper is to study the radial profiles and the corresponding scale-lengths, in the following figures we only show dust-mass profiles obtained with the full THEMIS model. We instead do not comment any further on the absolute values of the dust-mass surface density. A more complete analysis on the dust mass in DustPedia galaxies, including the contribution of grains from different environment to the SED composition, will be the subject of future publications from the collaboration.

\subsection{Stellar mass}

Stellar mass is one of the most fundamental parameters describing present-day galaxies. Gavazzi et al. (1996) and Gavazzi \& Scodeggio (1996) pointed out that the structure and star-formation history of disc galaxies are tightly linked with stellar mass. These findings can be extended to all morphologies as shown by Scodeggio et al. (2002). Bell \& de Jong (2000) pointed out that the mean stellar-mass density of a galaxy might be an even more basic parameter than the total stellar mass in determining the stellar populations in spiral galaxies. This conclusion has been confirmed by Kauffmann et al. (2003) and extended to all morphological types using more than 100000 galaxies from the SDSS.

To calculate the stellar-mass surface density we adopted two different methods. The first method makes use of the MIR images, specifically the IRAC 3.6 and $4.5 \mu \mathrm{m}$, and the prescription presented in Querejeta et al. (2015). They computed stellar mass maps using the Independent Component Analysis (ICA, Meidt et al. 2012) which is able to disentangle the contribution of the light from old stars and the dust emission, that can significantly contribute to the observed $3.6 \mu \mathrm{m}$ flux. Querejeta et al. (2015) also provided a relationship between the effective stellar mass-to-light ratio $(\mathrm{M} / \mathrm{L})$ and the observed [3.6] - [4.5] color (with 0.2 dex of uncertainties), calibrated using their optimal stellar mass estimates and that compares well with other relations in the literature (e.g., Eskew et al. 2012; Jarrett et al. 2013; McGaugh \& Schombert 2014).

The second method we adopted is based on the optical stellar images (SDSS and SINGS data) and the prescription of Zibetti et al. (2009). In that paper the authors presented a method to construct spatially resolved maps of stellar mass surface density in galaxies based on multi-band optical/near IR imaging, and the effective $\mathrm{M} / \mathrm{L}$ at each pixel is expressed as a function of one or two colors. We adopted the Table B1 of Zibetti et al. (2009) and the power-law fit to the $\mathrm{M} / \mathrm{L}$ ratio as a function of the optical color $(g-i)$ from the Bruzual \& Charlot (2003) models. The differences in stellar-mass surface density between the profiles obtained with Charlot \& Bruzual (2007) and Bruzual \& Charlot (2003) models are consistent within the errors, so we report only the results from the Charlot \& Bruzual (2007) models (Zibetti, priv. comm.). The calculation of the stellar-mass surface density from optical images can be done only for a fraction of galaxies of our sample: for nine galaxies, we adopted the Zibetti et al. (2009) procedure using the $(g-i)$ color, their SDSS images on the $g$ and $i$ bands being available, while for NGC 3621 and NGC 1097, we used the SINGS $B$ and $R$ images and the corresponding power-law fit.

As shown later (Figs. 3 and A.2), stellar mass profiles obtained from the optical and MIR images are in agreement for the majority of the nine face-on galaxies for which this comparison can be performed. For some galaxies (NGC 5194 (M 51), 


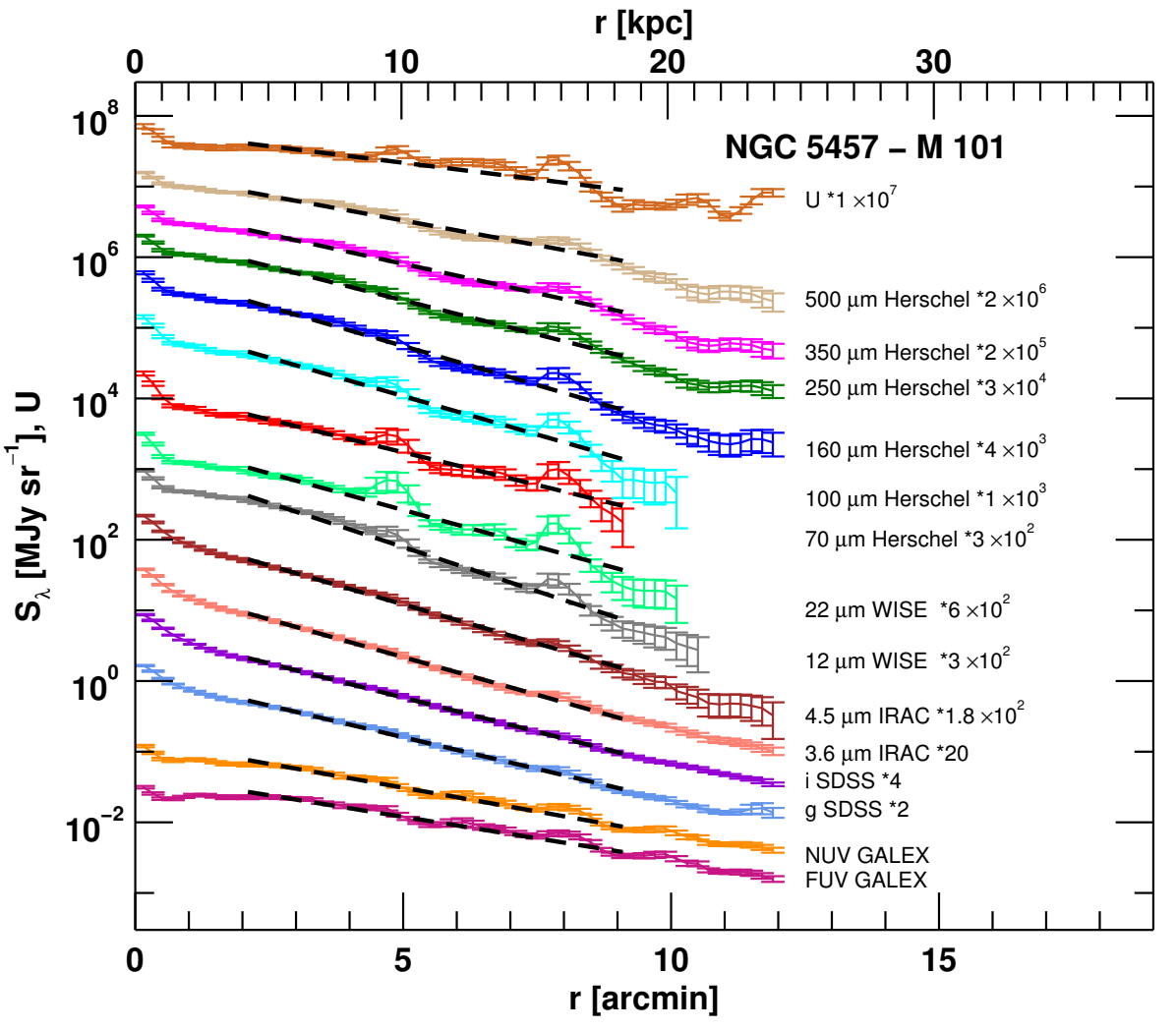

Fig. 2. Multi-wavelength surface-brightness profiles for the galaxy NGC 5457 (M 101), shown up to $r_{25}$. The profiles have been shifted for displaying purposes and the corresponding offsets are quoted next to each profile. The profiles are arranged in order of decreasing wavelength, from top to bottom. At the top, the interstellar radiation field intensity $U$, is shown, in dimensional units. The black dashed lines are exponential fits performed avoiding the central part of galaxies up to the maximum extension of the $70 \mu \mathrm{m}$ profile (shown in red).
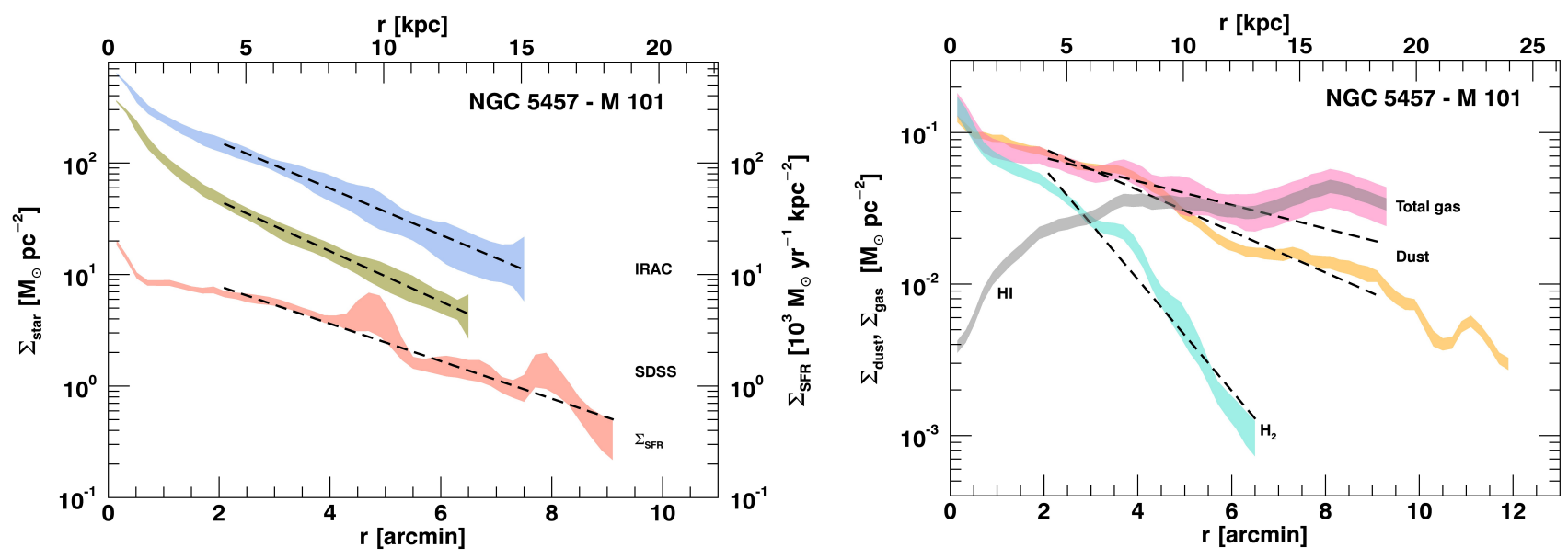

Fig. 3. Left panel: stellar-mass surface-density profiles from IRAC (blue) and from SDSS or SINGS (green), and SFR surface-density profiles (red) of the galaxy NGC 5457 (M 101), derived as described in Sects. 5.2 and 5.4, respectively. The black dashed lines are exponential fits performed avoiding the central part of galaxies in the same radius range as Fig. 2. Right panel: surface-density profiles for the mass of dust (orange), molecular gas (turquoise), atomic gas (gray), and total gas (pink) of the galaxy NGC 5457 (M 101). The gas-mass profiles have been corrected for DGR as described in Sect. 7.

NGC 5457 (M 101), and NGC 2403), the difference of profiles in the absolute scaling is notable and may be due to the adopted optical colors. Similar offsets in stellar profiles are also found in the analysis of the EAGLE simulations (Camps et al. 2016; Trayford et al. 2016).

We calculated the optical $(g-i)$ and MIR ([3.6]-[4.5]) colors using the aperture photometry for the nine face-on galaxies and we noticed that, while the MIR colors vary in the range $0.95-$ 0.97, the optical ones go from $\sim 0.74$ for NGC 5457 (M 101) up to $\sim 1.26$ for NGC 3031 (M 81). Our conclusion is that for the purpose of comparing the various profiles (dust, gas, stars) it is better to consider the IRAC images, weakly dependent on the dust with respect to the optical proxies, and the empirical relation of Querejeta et al. (2015), that compares well with the results obtained with other methods in the literature and that is available for our entire sample.

\subsection{Gas mass}

We derived the mass surface density of atomic gas $\left(\Sigma_{\mathrm{HI}}\right)$ from HI $21 \mathrm{~cm}$ line intensity $\left(I_{21} \mathrm{~cm}\right)$ images, under the assumption of optically thin HI emission, following:

$\Sigma_{\mathrm{HI}}=0.02 I_{21 \mathrm{~cm}}$, 
where $\Sigma_{\mathrm{HI}}$ is in units of $M_{\odot} \mathrm{pc}^{-2}$ and $I_{21 \mathrm{~cm}}$ in $\mathrm{K} \mathrm{km} \mathrm{s}^{-1}$ (e.g., Schruba et al. 2011).

We derived the mass surface density of molecular gas $\left(\Sigma_{\mathrm{H}_{2}}\right)$ from the ${ }^{12} \mathrm{CO}(1-0)$ or ${ }^{12} \mathrm{CO}(2-1)$ line intensity $\left(I_{\mathrm{CO}(1-0)}\right.$ or $\left.I_{\mathrm{CO}(2-1)}\right)$ images, based on the their availability, by adopting a constant value for the CO-to- $\mathrm{H}_{2}$ conversion factor expressed in the form $X_{\mathrm{CO}}\left(X_{\mathrm{CO}}=N\left(\mathrm{H}_{2}\right) / I_{\mathrm{CO}}\right.$ where $N\left(\mathrm{H}_{2}\right)$ is the molecular gas column density in $\mathrm{cm}^{-2}$ and $I_{\mathrm{CO}}$ is the $\mathrm{CO}$ line intensity in $\mathrm{K} \mathrm{km} \mathrm{s}^{-1}$ ). We assumed a value of $X_{\mathrm{CO}}=2.0 \times 10^{20} \mathrm{~cm}^{-2}$ $\left(\mathrm{K} \mathrm{km} \mathrm{s}^{-1}\right)^{-1}$ (e.g., Bolatto et al. 2013). This value of $X_{\mathrm{CO}}$ corresponds to a $\mathrm{CO}-$ to- $\mathrm{H}_{2}$ conversion factor expressed in terms of $\alpha_{\mathrm{CO}}\left(\alpha_{\mathrm{CO}}=M\left(\mathrm{H}_{2}\right) / L_{\mathrm{CO}}\right.$ where $M\left(\mathrm{H}_{2}\right)$ is the $\mathrm{H}_{2}$ mass in $M_{\odot}$ and $L_{\mathrm{CO}}$ the $\mathrm{CO}$ line luminosity in $\mathrm{K} \mathrm{km} \mathrm{s}^{-1} \mathrm{pc}^{2}$ ) of $\alpha_{\mathrm{CO}}=3.2 M_{\odot} \mathrm{pc}^{-2}\left(\mathrm{~K} \mathrm{~km} \mathrm{~s}^{-1}\right)^{-1}$ (Narayanan et al. 2012). $X_{\mathrm{CO}}$ and $\alpha_{\mathrm{CO}}$ are indeed related via $X_{\mathrm{CO}}\left[\left(\mathrm{K} \mathrm{km} \mathrm{s}^{-1}\right)^{-1}\right]=6.3 \times$ $10^{19} \alpha_{\mathrm{CO}}\left[M_{\odot} \mathrm{pc}^{-2}\left(\mathrm{~K} \mathrm{~km} \mathrm{~s}^{-1}\right)^{-1}\right]$. As is usual in the literature, we refer to the CO-to- $\mathrm{H}_{2}$ conversion factor both in terms of $X_{\mathrm{CO}}$ and $\alpha_{\mathrm{CO}}$ based on the context. In the case of ${ }^{12} \mathrm{CO}(2-1)$, we assumed a $\mathrm{CO}$ line ratio $I_{\mathrm{CO}(2-1)} / I_{\mathrm{CO}(1-0)}=0.7$, a typical value in HERACLES and other surveys (e.g., Leroy et al. 2009; Schruba et al. 2011). The derivation of $\Sigma_{\mathrm{H}_{2}}$ from $I_{\mathrm{CO}(1-0)}$ and $I_{\mathrm{CO}(2-1)}$, under the assumption of optically thick ${ }^{12} \mathrm{CO}$ emission, respectively, is through:

$\Sigma_{\mathrm{H}_{2}}=4.17 I_{\mathrm{CO}(1-0)}$

$\Sigma_{\mathrm{H}_{2}}=5.95 I_{\mathrm{CO}(2-1)}$,

where $\Sigma_{\mathrm{H}_{2}}$ is in units of $M_{\odot} \mathrm{pc}^{-2}$ and $I_{\mathrm{CO}(1-0)}$ and $I_{\mathrm{CO}(2-1)}$ in $\mathrm{K} \mathrm{km} \mathrm{s}^{-1}$. Equations (2)-(4) include a factor of 1.36 to account for heavy elements (see Schruba et al. 2011). When we refer to total gas (or the surface densities of mass of total gas), we consider the sum of atomic and molecular gas, $\Sigma_{\text {tot gas }}=\Sigma_{\mathrm{HI}}+\Sigma_{\mathrm{H} 2}$ (with the helium included).

\subsection{Star-formation rate}

We derived the surface density of the current SFR $\left(\Sigma_{\mathrm{SFR}}\right)$ combining GALEX-FUV and WISE $22 \mu \mathrm{m}$ according to Bigiel et al. (2008)'s calibration. They originally used the Spitzer $24 \mu \mathrm{m}$ emission to correct the dust attenuation of GALEX-FUV surface brightness. This composite FUV $+24 \mu \mathrm{m}$ SFR tracer, together with the $\mathrm{H} \alpha+24 \mu \mathrm{m}$ one (Kennicutt et al. 2007; Calzetti et al. 2007), has been extensively used for a large number of nearby galaxies (e.g., Rahman et al. 2011; Ford et al. 2013; Momose et al. 2013).

The $22 \mu \mathrm{m}$ flux densities can also be used to correct the FUV luminosity for dust attenuation since typical star-forming objects have a $22 \mu \mathrm{m}$ emission that should be comparable to, or slightly fainter than, the $24 \mu \mathrm{m}$ flux density (we refer e.g., to Hao et al. 2011; Cortese 2012; Eufrasio et al. 2014; Huang \& Kauffmann 2015 , for published applications). We estimated $\Sigma_{\text {SFR }}$ from the GALEX-FUV emission corrected by the WISE $22 \mu \mathrm{m}$ one using the relation presented by Bigiel et al. (2008) by replacing $24 \mu \mathrm{m}$ intensity with the $22 \mu \mathrm{m}$ one:

$\Sigma_{\mathrm{SFR}}=3.2 \times 10^{-3} \times I_{22}+8.1 \times 10^{-2} \times I_{\mathrm{FUV}}$,

where $\Sigma_{\mathrm{SFR}}$ is in units of $M_{\odot} \mathrm{yr}^{-1} \mathrm{kpc}^{-2}$, and $I_{22}$ and $I_{\mathrm{FUV}}$ are the $22 \mu \mathrm{m}$ and FUV intensities, respectively, in units of $\mathrm{MJy} \mathrm{sr}^{-1}$. When the $22 \mu \mathrm{m}$ information is not available $\left(I_{22}=0\right)$, Eq. (5) reduces to the FUV-SFR calibration by Salim et al. (2007). This calibration of $\Sigma_{\mathrm{SFR}}$ is based on the initial mass function (IMF) from Calzetti et al. (2007), which is the default IMF in STARBURST99 (Leitherer et al. 1999) consisting of two power laws, with slope -1.3 in the range $0.1-0.5 M_{\odot}$ and slope -2.3 in the range $0.5-120 M_{\odot}$. To convert to the truncated Salpeter (1955) IMF adopted by, for example, Kennicutt $(1989,1998$ a) or Kennicutt et al. (2007), one should multiply our $\Sigma_{\text {SFR }}$ by a factor of 1.59 .

One caveat in the adopted SFR calibration is that both the $22 \mu \mathrm{m}$ and FUV emission might have a contribution from stars that are too old to be associated with recent SF (e.g., Kennicutt et al. 2009). FUV is a tracer sensitive to SF on a timescale of $\sim 100$ Myr (e.g., Kennicutt 1998a; Calzetti et al. 2005; Salim et al. 2007), while $22 \mu$ m emission, predominantly due to dust-heating by UV photons from bright young stars, is sensitive to a SF timescale $<10$ Myr (e.g., Calzetti et al. 2005, 2007; Pérez-González et al. 2006). Old stars ( $\gtrsim 1$ Gyr) are fainter but redder and so emit relatively stronger at $3.6 \mu \mathrm{m}$. A method to mitigate for the old stars is indeed based on the determination of FUV and $24 \mu \mathrm{m}$ emission with respect to the $3.6 \mu \mathrm{m}$ one in regions where SF has ceased, and use this to remove the component of the FUV and $24 \mu \mathrm{m}$ emission coming from old stars (e.g., Leroy et al. 2008; Ford et al. 2013). However, Leroy et al. (2008) found that both FUV and $24 \mu \mathrm{m}$ bands do appear to be dominated by a young stellar population almost everywhere in their sample of 23 galaxies, including spirals and irregulars, 9 of which are in common with our sample. For this reason we did not apply the correction for the old star component in our sample of spirals. Vice versa, this correction might be mandatory in the case of elliptical galaxies, dominated by pure populations of very old stars with no evidence of younger stars. Anyway, since we are not interested in absolute values of the SFR, but on the radial profiles, we do not expect significant differences on the scale-length values derived from profiles.

\section{Surface-brightness profiles, and mass and star-formation rate surface-density profiles}

The collected data and the analysis of the surface-brightness profiles allowed us to study how dust emission relates to the distribution of stellar light. By examining the shape of the surface brightness profiles at each wavelength, we can make a comparison that is independent of the absolute calibration.

Radial surface-brightness profiles were extracted from the dust (Herschel and WISE) and stellar (GALEX, SDSS, SINGS, and IRAC) images. We used elliptical averaging, a widely used traditional technique which was recently proven to fare as well as more sophisticated approaches (Peters et al. 2017). The adopted centers, position angles (PA), inclination angles $(i)$, and minorto-major axis ratios $(b / a)$ are listed in Table 1 . The PA and $i$ angles have been determined kinematically and extracted from references listed in Table 1. The width of each annulus is the same as the pixel size and the radial profile extraction extends up to the largest radius for which the surface brightness is larger than $2 \sigma$. Uncertainties in the surface brightnesses as a function of radius were calculated as the quadrature sum of the standard deviation of the mean along the elliptical isophotes and of the uncertainties on the sky subtraction.

Besides the dust surface-density profile, whose derivation we describe in Sect. 5.1, we also produced mass surface-density profiles of the stars and gas (atomic, molecular, and total), and SFR surface-density profiles, from the maps derived as in Sects. 5.2-5.4, respectively. Uncertainties in the mass profiles as a function of radius were calculated as the quadrature sum of the standard deviation along the elliptical isophotes and the uncertainty on the mass determination. Uncertainties in the $\Sigma_{\mathrm{SFR}}$ profiles as a function of radius were calculated as the quadrature sum of the variation along the elliptical isophotes. 
Table 4. Inner and outer radii used for exponential fitting for each sample galaxy.

\begin{tabular}{lccccccc}
\hline \hline Galaxy & $\begin{array}{c}r_{\text {in }} \\
{\left[^{\prime}\right]}\end{array}$ & $\begin{array}{c}r_{\text {in }} \\
{[\mathrm{kpc}]}\end{array}$ & $r_{\text {in }} / r_{25}$ & $r_{\text {out }}$ (prof.) & $\begin{array}{c}r_{\text {out }} \\
{\left[^{\prime}\right]}\end{array}$ & $\begin{array}{c}r_{\text {out }} \\
{[\mathrm{kpc}]}\end{array}$ & $r_{\text {out }} / r_{25}$ \\
\hline NGC 5457 (M 101) & 2.1 & 4.3 & 0.18 & $70 \mu \mathrm{m}$ & 9.1 & 18.5 & 0.76 \\
NGC 3031 (M 81) & 5.1 & 5.5 & 0.47 & $70 \mu \mathrm{m}$ & 9.3 & 10.0 & 0.85 \\
NGC 2403 & 4.1 & 4.2 & 0.41 & $12 \mu \mathrm{m}$ & 8.5 & 8.7 & 0.85 \\
IC 342 & 2.1 & 1.9 & 0.21 & $70 \mu \mathrm{m}$ & 9.9 & 8.9 & 0.99 \\
NGC 300 & 3.5 & 2.0 & 0.36 & $100 \mu \mathrm{m}$ & 7.1 & 4.1 & 0.73 \\
NGC 5194 (M 51) & 2.1 & 4.8 & 0.30 & $*$ & 3.9 & 8.9 & 0.56 \\
NGC 5236 (M 83) & 2.1 & 4.0 & 0.31 & $70 \mu \mathrm{m}$ & 5.7 & 10.8 & 0.85 \\
NGC 1365 & 1.1 & 5.6 & 0.18 & $70 \mu \mathrm{m}$ & 3.5 & 18.0 & 0.58 \\
NGC 5055 (M 63) & 0.9 & 2.1 & 0.15 & $70 \mu \mathrm{m}$ & 4.7 & 11.3 & 0.80 \\
NGC 6946 & 1.1 & 1.8 & 0.19 & $12 \mu \mathrm{m}$ & 3.1 & 5.1 & 0.54 \\
NGC 925 & 1.1 & 2.8 & 0.21 & $70 \mu \mathrm{m}$ & 4.7 & 11.8 & 0.88 \\
NGC 1097 & 1.0 & 6.3 & 0.21 & $100 \mu \mathrm{m}$ & 4.5 & 25.7 & 0.86 \\
NGC 7793 & 1.1 & 1.2 & 0.21 & $70 \mu \mathrm{m}$ & 4.1 & 4.5 & 0.78 \\
NGC 628 (M 74) & 1.1 & 2.9 & 0.22 & $70 \mu \mathrm{m}$ & 4.1 & 10.8 & 0.82 \\
NGC 3621 & 1.1 & 2.2 & 0.23 & $70 \mu \mathrm{m}$ & 4.5 & 9.1 & 0.92 \\
NGC 4725 & 2.1 & 8.3 & 0.43 & $70 \mu \mathrm{m}$ & 4.3 & 17.1 & 0.88 \\
NGC 3521 & 1.1 & 3.9 & 0.27 & $70 \mu \mathrm{m}$ & 5.3 & 18.5 & 1.27 \\
NGC 4736 (M 94) & 0.9 & 1.4 & 0.23 & $70 \mu \mathrm{m}$ & 3.3 & 5.0 & 0.85 \\
\hline Mean & & & $0.27 \pm 0.09$ & & & & $0.82 \pm 0.17$ \\
\hline
\end{tabular}

Notes. * For NGC 5194 (M 51) the exponential fits stop at a radius of 3.9, lower than $r_{\text {out }}(70 \mu \mathrm{m}$ ), because its interacting companion (NGC 5195) affects surface-brightness profiles at larger radii.

\subsection{Exponential disks and scale-lengths}

We fitted the surface-brightness profiles of the tracers of dust and stars, the mass surface-density profiles of dust, stars, molecular gas, and total gas, and the SFR surface-density profiles with a simple exponential curve, $S=S_{0} \exp \left(-h / h_{0}\right)$, where $h_{0}$ is the scale-length, and $S_{0}$ is the surface brightness (or mass/SFR surface density) at the radius $h=0$ (we do not analyze this quantity in the present work). The exponential decline with the galactic radius is generally a good approximation for the surface-brightness profiles of stellar disks (van der Kruit \& Freeman 2011) and has been extended to the description of dust and molecular gas disks (e.g., Xilouris et al. 1999; Regan et al. 2001).

We excluded from the fit the central part of the surfacebrightness profiles where the emission might be dominated, at least for the stellar component, by the presence of a spheroidal bulge. For each galaxy we determined the starting/inner radius $\left(r_{\text {in }}\right)$ for the fit by eye and once selected this we adopted the same $r_{\text {in }}$ for all profiles of that galaxy. Despite the choice tailored to each object, the resulting $r_{\text {in }}$ are not too dissimilar, with an average ratio $r_{\text {in }} / r_{25}=0.27$ and a $1-\sigma$ scatter of 0.09 . For all profiles of a given galaxy, the exponential fit extends up to the faintest emission level at $70 \mu \mathrm{m}$ surface-brightness profile (outer radius, $\left.r_{\text {out }}(70 \mu \mathrm{m})\right)$, or to those at 12 and $100 \mu \mathrm{m}$ when the $70 \mu \mathrm{m}$ profile is not available (NGC 300) or it is more extended than the $12 \mu \mathrm{m}$ (NGC 2403, NGC 6946) and $100 \mu \mathrm{m}$ (NGC 1097) profiles. For NGC 5194 (M 51) the exponential fits stop at a radius lower than $r_{\text {out }}(70 \mu \mathrm{m})$ because its interacting companion (NGC 5195) affects surface-brightness profiles at larger radii. Again, the resulting $r_{\text {out }}$ are rather uniform, with an average ratio $r_{\text {out }} / r_{25}=0.82$ and a $1-\sigma$ scatter of 0.17 . The small differences in the ranges that are used for the fitting should not be of concern, in particular when the ratios between scale-lengths are analyzed. Table 4 collects the inner and outer radii used for exponential fitting for each sample galaxy. Though we do not perform a true bulge-disk decomposition, the derived scale-lengths still provide a good characterization of the gradients of the various disk components we consider.

An extra source of photons such as an AGN, present in the nuclei of some sample galaxies (Table 1), which basically emits in the whole spectral range we are taking into account, could be, in principle, influencing the observed profiles. There are several reasons why we believe this is not affecting our analysis:

- the accretion disk mainly emits UV and optical radiation, which is very efficiently absorbed by dust (and gas) within the central region of the galaxy;

- given the low luminosities of the AGN in the sample, possible radiation leaks to outer regions are negligible with respect to the luminosity of stars at similar wavelengths;

- the dusty torus, which is instead heated by the accretion disk emission, and would hence emit in the NIR and MIR, is believed to be very weak at these luminosities, or even absent (e.g., González-Martín et al. 2015);

- the central regions ( $\lesssim 1-2 \mathrm{kpc}$, see Table 4$)$, which is where a possible influence by AGN emission could be spotted, are not considered in our analysis.

Figure 2 shows the example of surface-brightness and interstellar-radiation-field profiles and exponential fits for the galaxy NGC 5457 (M 101) and Fig. A.1 displays those of the entire face-on DustPedia sample. The profiles are shown up to $r_{25}$. Table 5 collects the exponential scale-lengths derived for the multi-wavelength surface-brightness profiles.

Also the stellar mass surface density profiles, SFR surfacedensity profiles, and mass surface density profiles of dust, molecular gas, and total gas have been fitted within the same radius as that of the individual surface-brightness profiles. Figure 3 shows these profiles for the galaxy NGC 5457 (M 101), Fig. A.2 displays those of the entire face-on DustPedia sample, and Table 6 collects the corresponding exponential scale-lengths. The gas 


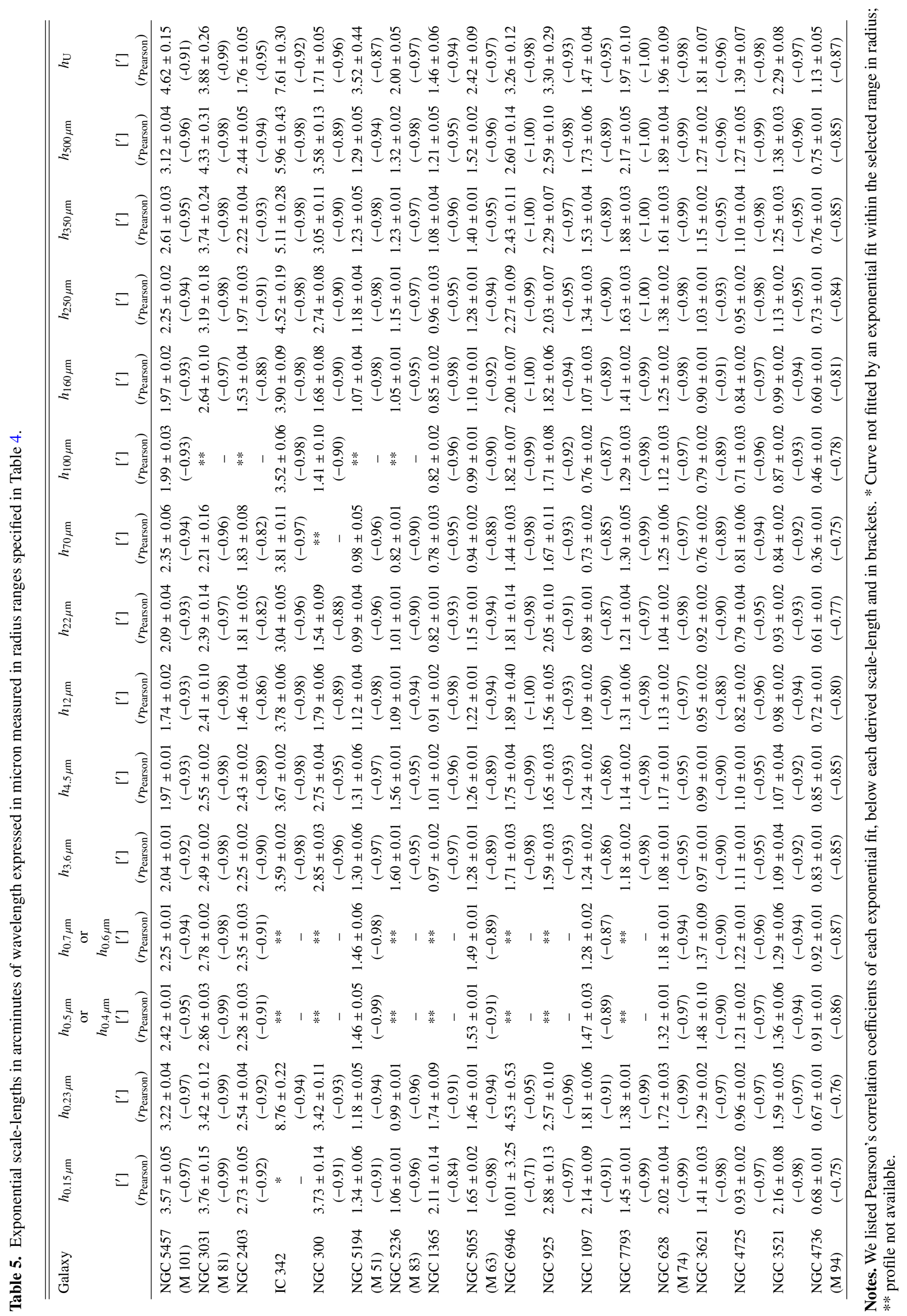


V. Casasola et al.: Scale-lengths in DustPedia

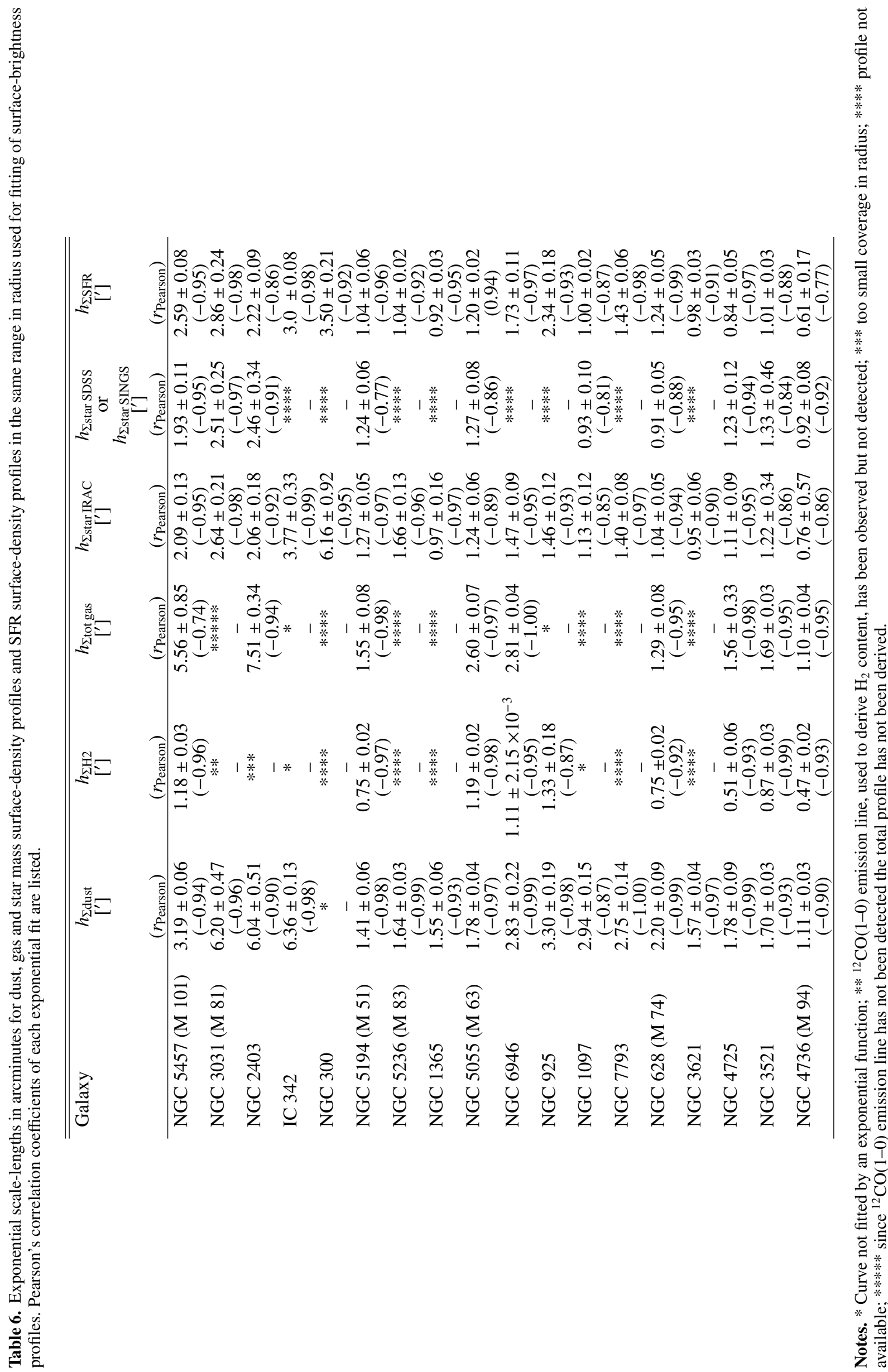




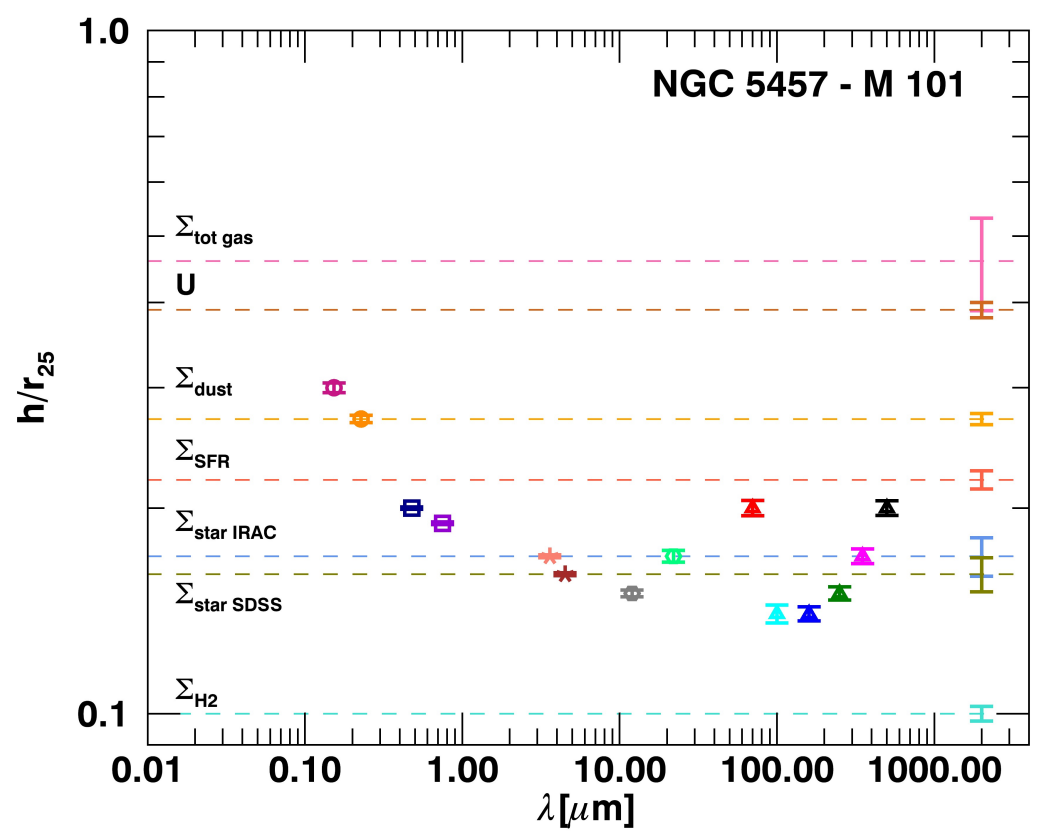

Fig. 4. Scale-length fitted to the surface-brightness profiles from UV to sub-mm and normalized with respect to $r_{25}, h / r_{25}$, as a function of wavelength for the galaxy NGC 5457 (M 101). The scale-lengths of mass (of dust, gas, and stars) and SFR surface-density profiles and the scalelength of $U$ profile, normalized with respect to $r_{25}$, are plotted as horizontal lines because they are not associated with a wavelength. They and the corresponding error bars are drawn at $\lambda=2000 \mu \mathrm{m}$.

mass surface density profiles shown in Figs. 3 and A.2 are corrected for radial variations in DGR and $\alpha_{\mathrm{CO}}$ conversion factor, both properties derived for each single galaxy according to the prescription described in Sect. 7. The adopted values do not affect the derivation of the scale-length for molecular gas. We do not fit the HI gas surface density profiles, as these do not generally follow an exponential distribution. In general, the difference between $\mathrm{HI}$ and $\mathrm{H}_{2}$ (or $\mathrm{CO}$ ) radial distributions in galaxies is striking (e.g., Wong \& Blitz 2002; Bigiel et al. 2008) and HI gas alone extends far beyond the "optical" disk, sometimes in average by a factor 2 to $4\left(R_{\mathrm{HI}} \sim 2-4 R_{\mathrm{opt}}\right)$. The HI gas has very often a small deficiency in the center, possibly due to the transformation of the atomic gas in molecular phase in the denser central parts of galaxies. Bigiel et al. (2008) indeed find that $\Sigma_{\mathrm{HI}}$ saturates at a surface density of $\approx 9 M_{\odot} \mathrm{pc}^{-2}$, and gas in excess of this value is in the molecular phase both in spirals and in HI-dominated galaxies. This is the most common trend in galaxies, where the $\mathrm{HI}$ and $\mathrm{CO}$ distributions appear complementary, but it is not the general case, and all possibilities have been observed, including a central gaseous hole, both in $\mathrm{CO}$ and $\mathrm{HI}$ (like in the Milky Way, M 31, NGC 3031 (M 81), and NGC 3147 in Misiriotis et al. 2006; Nieten et al. 2006; Casasola et al. 2007, 2008, respectively).

\subsection{Results}

Figures 4 and A.3 show the scale-lengths fitted to the surface-brightness profiles from UV to sub-mm, normalized with respect to $r_{25}\left(h / r_{25}\right)$, as a function of wavelength, for NGC 5457 (M 101) and the entire face-on DustPedia sample, respectively. Together with the scale-lengths of surfacebrightness profiles, we also plotted those of $U$ profiles, and of mass and SFR surface-density profiles. Since $U$ profiles and mass and SFR surface density profiles are not associated with a given wavelength, we plotted their scale-lengths marked with horizontal lines and the corresponding error bars are drawn at $\lambda=2000 \mu \mathrm{m}$.

The analysis performed for the whole sample shows a variety of behaviors in terms of exponential scale-lengths despite the fact that the investigated galaxies belong to the same subclass of objects (i.e., nearby, face-on, large, spiral galaxies) and that all images have been homogeneously treated. Therefore, each galaxy has its own trend of scale-lengths. This is likely due to the fact that the derived scale-lengths are affected by the peculiarities of a given galaxy, like, for example, the local maxima in the spiral arms. Nevertheless, we identified some common behaviors in our sample: they are highlighted in Fig. 5, showing the mean $r_{25}$-normalized scale-lengths, $\left\langle h / r_{25}\right\rangle$, over the whole sample, where differences due to the peculiarities of individual galaxies are all smeared out. The mean values of $r_{25}$-normalized scale-lengths and scale-length ratios for selected bands are collected in Table 7.

From Fig. 5 it can be seen that the mean $r_{25}$-normalized stellar surface-brightness scale-lengths decrease from the UV to the NIR by a factor $\sim 1.5$, with values of $\left\langle h_{\mathrm{NUV}} / r_{25}\right\rangle \sim 0.34$ and $\left\langle h_{4.5 \mu \mathrm{m}} / r_{25}\right\rangle \sim 0.24$, respectively. This decrease continues in the MIR dust-dominated bands and the mean $r_{25}$-normalized scalelength reaches a minimum at $70 \mu \mathrm{m}$ of $\sim 0.20$. From $70 \mu \mathrm{m}$ to the sub-mm, instead, there is a steady increase up to the $500 \mu \mathrm{m}$ scale-length with a value of $\left\langle h_{500 \mu \mathrm{m}} / r_{25}\right\rangle \sim 0.32$. There are individual variations to these common trends: the UV scale-lengths in some galaxies are smaller than those in the optical and NIR (e.g., NGC 5194 (M 51)); in a few cases they are much larger than in the common trends (e.g., NGC 6946, where unfortunately the intermediated optical SDSS/SINGS data are absent); the steady increase in the FIR can start at wavelengths longer than $70 \mu \mathrm{m}$ (e.g., NGC 5457 (M 101)).

The scale-lengths of the dust-mass surface-density profiles are larger than those of the surface-brightness profiles at $500 \mu \mathrm{m}$ $\left(\left\langle h_{\Sigma \text { dust }} / h_{500 \mu \mathrm{m}}\right\rangle \sim 1.3\right)$. They are generally larger than most of 


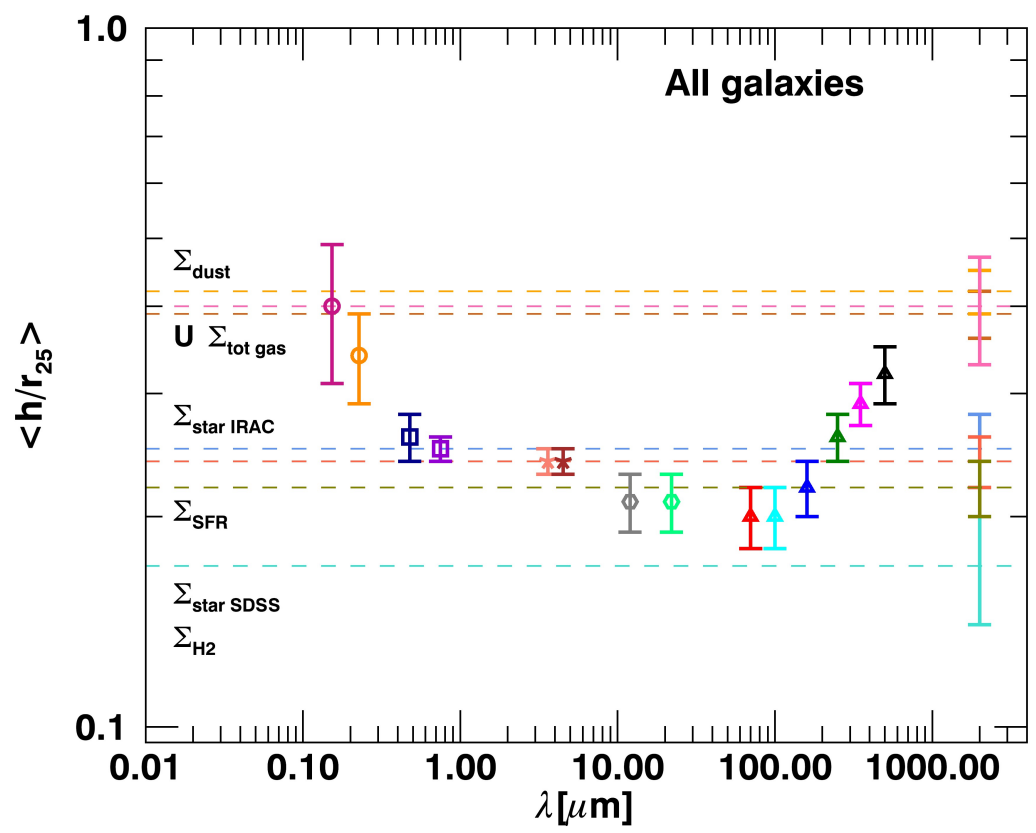

Fig. 5. Mean scale-length normalized with respect to $r_{25}$ of all sample galaxies as a function of wavelength. Scale-lengths of mass (of dust, gas, and stars) and SFR surface-density profiles, the scale-length of $U$ profile, and the corresponding error bars are drawn as in Fig. 4.

the other surface-brightness scale-lengths, and than those of the stellar-mass surface-density profiles. On average, the dust-mass surface-density scale-length is about 1.8 times the scale-length of the stellar-mass surface-density derived from IRAC data and of the $3.6 \mu \mathrm{m}$ surface brightness. Only for NGC 300 is the dustmass surface-density gradient so flat that the exponential profile was not fitted. Only the average FUV scale-length can be as large as the dust-mass surface-density gradient, though the scatter in the former is large. Also, the interstellar-radiation-field intensity has a gradient comparable to that of the dust mass. SDSS and IRAC stellar-mass profiles have comparable scale-lengths, and similar to those of the corresponding surface-brightness profiles from which they have been derived ( $g$ and $i$ bands from SDSS, $B$ and $R$ bands from SINGS, and 3.6 and $4.5 \mu \mathrm{m}$ bands from IRAC). The scale-lengths of the SFR surface density are comparable to those of the stellar $3.6 \mu \mathrm{m}$ emission and of the stellarmass distribution.

As for the gas, the $\mathrm{H}_{2}$ surface-density profiles have typically smaller scale-lengths than the NIR surface-brightness profiles (e.g., $\left\langle h_{\Sigma \mathrm{H} 2} / h_{12 \mu \mathrm{m}}\right\rangle \sim 0.8$ ) and than the IRAC stellar-mass surface-density profiles, and thus, on average, a factor $\sim 2.3$ smaller than the dust mass ones. We stress that these results are based on $\mathrm{H}_{2}$ gas profiles typically less extended than the others. The total gas-mass surface-density profiles, instead, have scale-lengths slightly larger than the dust $\left(\left\langle h_{\Sigma \text { tot gas }} / h_{\Sigma \text { dust }}\right\rangle \sim 1.1\right)$, though the result is influenced by the assumed value for $\alpha_{\mathrm{CO}}$, and thus by the assumed relative contribution of atomic and molecular components to the total. We will discuss this issue further in the following section.

We also studied the trends of the scale-lengths as a function of Hubble stage $T$. In general, the scatter is large and apparent trends are always feeble, within the scatter. Figure 6 shows the trends for exponential scale-length ratios that are less weak. The ratio between the scale-lengths of the Herschel bands and the $3.6 \mu \mathrm{m}$ scale-length tends to increase from earlier to later types, with the stronger trend being that for $h_{70 \mu \mathrm{m}} / h_{3.6 \mu \mathrm{m}}$ (panel a in Fig. 6). The value for $h_{70 \mu \mathrm{m}}$ instead, tends to be smaller than that at longer sub-mm wavelength and shown by the decreasing $h_{250 \mu \mathrm{m}} / h_{70 \mu \mathrm{m}}$ with increasing $T$ (panel b). Among the surfacedensity ratios, only $h_{\Sigma \mathrm{SFR}} / h_{3.6 \mu \mathrm{m}}$ shows a weak trend of increasing SFR scale-length, with respect to stars, when moving to later types (panel c).

\subsection{Discussion and comparison with the literature}

The mean stellar scale-lengths we find in this work (e.g., $\left.h_{3.6 \mu \mathrm{m}} / r_{25} \sim 0.24\right)$ are consistent within the scatter to those found from detailed two-dimensional bulge-disk decompositions of spiral galaxy images (0.25-0.30, e.g., Giovanelli et al. 1994; Moriondo et al. 1998; Hunt et al. 2004). Also, the decrease in the surface-brightness scale-lengths from the UV to the NIR is in line with several previous works in the literature (Peletier et al. 1994; de Jong 1996; Pompei \& Natali 1997; Moriondo et al. 1998; MacArthur et al. 2003; Möllenhoff 2004; Taylor et al. 2005; Fathi et al. 2010). The color gradient has been explained with an intrinsic gradient in the stellar populations, resulting in a blueing of the disks at a greater distance from the center (de Jong 1996; Cunow 2004; MacArthur et al. 2004), compatible with an inside-out formation scenario (Muñoz-Mateos et al. 2007). Other authors, instead, claim that the effect is mostly due to preferential reddening in the inner regions of a galaxy (Beckman et al. 1996; Pompei \& Natali 1997; Möllenhoff et al. 2006) or that dust has, at least, a significant role together with stars in shaping the gradient (Muñoz-Mateos et al. 2011).

According to Alton et al. (1998), we interpret the steady increase in the surface-brightness scale-length from 70 to $500 \mu \mathrm{m}$ as the result of a cold-dust temperature gradient with galactocentric distance combined with a scale-length of the dust disk larger than the stellar (we also refer to Davies et al. 1999). Such a cold-dust temperature gradient is consistent with a dust heating by a diffuse interstellar radiation field gradually decreasing with galactocentric distance, while a more clumpy stellar emission 
Table 7. Mean values over the entire galaxy sample of the $r_{25^{-}}$ normalized scale-lengths $\left(h / r_{25}\right)$ of $U$, of the surface-brightness profiles from FUV to $500 \mu \mathrm{m}$, and of the surface-density profiles of mass (of dust, gas, and star) and SFR.

\begin{tabular}{|c|c|c|}
\hline Ratio & Value & No. of galaxies \\
\hline$\left\langle h_{U} / r_{25}\right\rangle$ & $0.39 \pm 0.03$ & 18 \\
\hline$\left\langle h_{\mathrm{FUV}} / r_{25}\right\rangle$ & $0.40 \pm 0.09$ & 17 \\
\hline$\left\langle h_{\mathrm{NUV}} / r_{25}\right\rangle$ & $0.34 \pm 0.05$ & 18 \\
\hline$\left\langle h_{0.5 \mu \mathrm{m}} / r_{25}\right\rangle$ & $0.26 \pm 0.02$ & 11 \\
\hline$\left\langle h_{0.7 \mu \mathrm{m}} / r_{25}\right\rangle$ & $0.25 \pm 0.01$ & 11 \\
\hline$\left\langle h_{3.6 \mu \mathrm{m}} / r_{25}\right\rangle$ & $0.24 \pm 0.01$ & 18 \\
\hline$\left\langle h_{4.5 \mu \mathrm{m}} / r_{25}\right\rangle$ & $0.24 \pm 0.01$ & 18 \\
\hline$\left\langle h_{12 \mu \mathrm{m}} / r_{25}\right\rangle$ & $0.21 \pm 0.02$ & 18 \\
\hline$\left\langle h_{22 \mu \mathrm{m}} / r_{25}\right\rangle$ & $0.21 \pm 0.02$ & 18 \\
\hline$\left\langle h_{70 \mu \mathrm{m}} / r_{25}\right\rangle$ & $0.20 \pm 0.02$ & 17 \\
\hline$\left\langle h_{100 \mu \mathrm{m}} / r_{25}\right\rangle$ & $0.20 \pm 0.02$ & 14 \\
\hline$\left\langle h_{160 \mu \mathrm{m}} / r_{25}\right\rangle$ & $0.22 \pm 0.02$ & 18 \\
\hline$\left\langle h_{250 \mu \mathrm{m}} / r_{25}\right\rangle$ & $0.26 \pm 0.02$ & 18 \\
\hline$\left\langle h_{350 \mu \mathrm{m}} / r_{25}\right\rangle$ & $0.29 \pm 0.02$ & 18 \\
\hline$\left\langle h_{500 \mu \mathrm{m}} / r_{25}\right\rangle$ & $0.32 \pm 0.03$ & 18 \\
\hline$\left\langle h_{\Sigma \text { dust }} / r_{25}\right\rangle$ & $0.42 \pm 0.03$ & 17 \\
\hline$\left\langle h_{\Sigma \mathrm{H} 2} / r_{25}\right\rangle$ & $0.17 \pm 0.03$ & 9 \\
\hline$\left\langle h_{\Sigma \text { tot gas }} / r_{25}\right\rangle$ & $0.40 \pm 0.07$ & 9 \\
\hline$\left\langle h_{\Sigma \text { star IRAC }} / r_{25}\right\rangle$ & $0.25 \pm 0.03$ & 18 \\
\hline$\left\langle h_{\Sigma \text { star SDSS }} / r_{25}\right\rangle$ & $0.22 \pm 0.02$ & 10 \\
\hline$\left\langle h_{\Sigma \mathrm{SFR}} / r_{25}\right\rangle$ & $0.24 \pm 0.02$ & 18 \\
\hline$\left\langle h_{12 \mu \mathrm{m}} / h_{3.6 \mu \mathrm{m}}\right\rangle$ & $0.90 \pm 0.03$ & 18 \\
\hline$\left\langle h_{70 \mu \mathrm{m}} / h_{3.6 \mu \mathrm{m}}\right\rangle$ & $0.84 \pm 0.05$ & 17 \\
\hline$\left\langle h_{160 \mu \mathrm{m}} / h_{3.6 \mu \mathrm{m}}\right\rangle$ & $0.91 \pm 0.05$ & 18 \\
\hline$\left\langle h_{250 \mu \mathrm{m}} / h_{3.6 \mu \mathrm{m}}\right\rangle$ & $1.07 \pm 0.05$ & 18 \\
\hline$\left\langle h_{500 \mu \mathrm{m}} / h_{3.6 \mu \mathrm{m}}\right\rangle$ & $1.34 \pm 0.07$ & 18 \\
\hline$\left\langle h_{160 \mu \mathrm{m}} / h_{70 \mu \mathrm{m}}\right\rangle$ & $1.14 \pm 0.05$ & 17 \\
\hline$\left\langle h_{250 \mu \mathrm{m}} / h_{70 \mu \mathrm{m}}\right\rangle$ & $1.32 \pm 0.06$ & 17 \\
\hline$\left\langle h_{500 \mu \mathrm{m}} / h_{160 \mu \mathrm{m}}\right\rangle$ & $1.48 \pm 0.05$ & 18 \\
\hline$\left\langle h_{\Sigma \text { dust }} / h_{3.6 \mu \mathrm{m}}\right\rangle$ & $1.77 \pm 0.12$ & 17 \\
\hline$\left\langle h_{\Sigma \text { dust }} / h_{500 \mu \mathrm{m}}\right\rangle$ & $1.33 \pm 0.09$ & 17 \\
\hline$\left\langle h_{\Sigma \text { dust }} / h_{\mathrm{H} 2}\right\rangle$ & $2.27 \pm 0.30$ & 9 \\
\hline$\left\langle h_{\Sigma \text { dust }} / h_{\Sigma \text { star IRAC }}\right\rangle$ & $1.80 \pm 0.13$ & 17 \\
\hline$\left\langle h_{\Sigma \mathrm{H} 2} / h_{3.6 \mu \mathrm{m}}\right\rangle$ & $0.73 \pm 0.11$ & 9 \\
\hline$\left\langle h_{\Sigma \mathrm{H} 2} / h_{12 \mu \mathrm{m}}\right\rangle$ & $0.78 \pm 0.14$ & 9 \\
\hline$\left\langle h_{\Sigma \text { tot gas }} / h_{\Sigma \text { dust }}\right\rangle$ & $1.11 \pm 0.16$ & 9 \\
\hline$\left\langle h_{\Sigma \mathrm{star} \text { IRAC }} / h_{3.6 \mu \mathrm{m}}\right\rangle$ & $1.06 \pm 0.07$ & 18 \\
\hline$\left\langle h_{\Sigma \mathrm{SFR}} / h_{3.6 \mu \mathrm{m}}\right\rangle$ & $0.99 \pm 0.05$ & 18 \\
\hline
\end{tabular}

Notes. Other mean ratios between scale-lengths are collected.

(and dust absorption/emission) would have resulted in flatter FIR/sub-mm profiles (as shown, though for edge-on systems, by Bianchi 2008). The diffuse field could arise for a widespread component of old stars, and thus the derived trend might enforce the idea that dust heating and emission are not related to recent SF. However, it could also arise from younger stars, if parental clouds have a sufficient leakage of radiation. There might not be a simple answer to the question: indeed, the analysis of submm color-maps by Bendo et al. (2015) has shown that old and young stars might contribute in different ways to dust emission at different wavelengths, depending on the particular situation of each galaxy. The flat trend in the derived radiation field, with $h_{U} \approx h_{\mathrm{FUV}}$, seems to suggest a dominant contribution of SF to dust heating. The result is puzzling, though, as one would expect a non negligible contribution to heating from older stars, and thus a scale-length in between $h_{\mathrm{FUV}}$ and $h_{3.6 \mu \mathrm{m}}$; the FUV

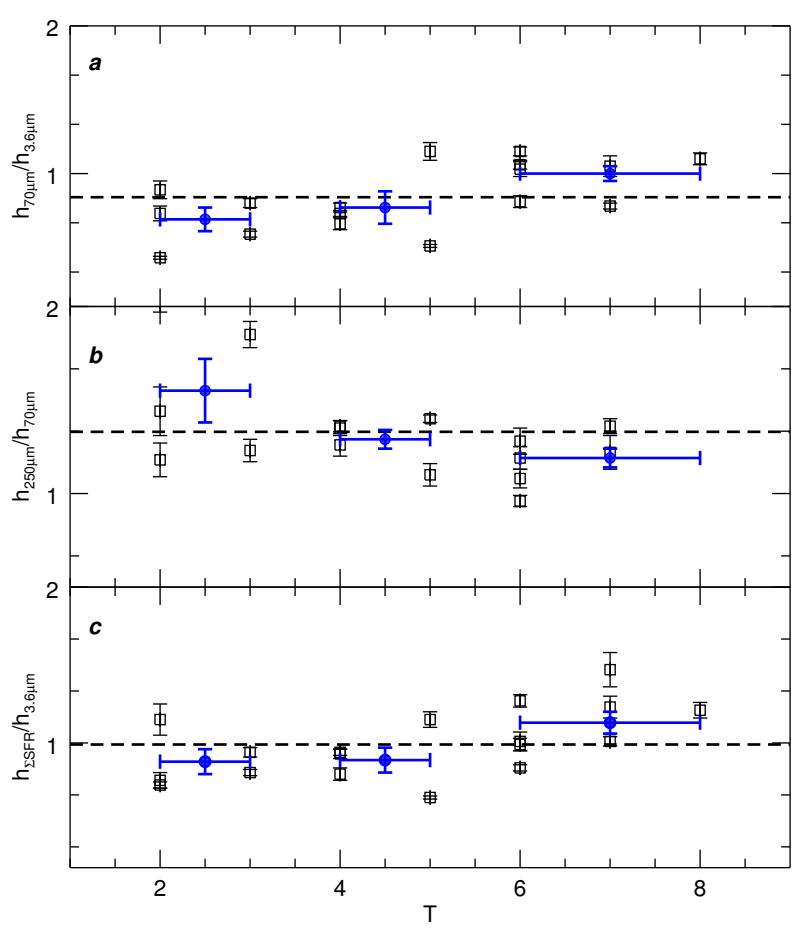

Fig. 6. Ratios of the exponential scale-lengths as a function of Hubble stage $T: a: 70 \mu \mathrm{m} / 3.6 \mu \mathrm{m}, b: 250 \mu \mathrm{m} / 3.6 \mu \mathrm{m}, c: \Sigma_{\mathrm{SFR}} / 3.6 \mu \mathrm{m}$. In each panel, the (blue) large circles with error bars are the means over small ranges of Hubble stages $(2-3,4-5,6-8)$ as indicated by the horizontal error bars, while the vertical error bars are the standard deviations. The horizontal dashed lines show the mean scale-lengths by averaging all galaxies.

gradient might also be significantly flattened by dust extinction. We cannot exclude that the result is due to the effect of systematic error in the modeling due to the non-linear dependence of the modeled SED on $U$, combined with the temperature mixing along the line of sight. We only note that we found little differences in $h_{U}$ estimates if we used the pixel-by-pixel procedure for SED fitting, which should be less affected by temperature mixing (see Sect. 5.1).

For the dust-emission surface-brightness profiles we find trends similar to those of Alton et al. (1998) (i.e., a steady increase of the scale-length for larger wavelengths), though with different and much less extreme scale-length ratios, as a result of the dramatic improvement in resolution from the IRAS/ISO data to the Herschel ones. While Alton et al. (1998) found that the $200 \mu \mathrm{m}$ scale-length was about $30 \%$ larger than that in the $B$-band (with a mean scale-length ratio of $\sim 1.3$ ), we derive here that the 160 and $250 \mu \mathrm{m}$ scale-lengths are of the same order as the $3.6 \mu \mathrm{m}$ scale-length $\left(\left\langle h_{160 \mu \mathrm{m}} / h_{3.6 \mu \mathrm{m}}\right\rangle \sim 0.9\right.$, $\left.\left\langle h_{250 \mu \mathrm{m}} / h_{3.6 \mu \mathrm{m}}\right\rangle \sim 1.1\right)$ and thus are smaller than those in the $B$ band, for the wavelength trends we discussed. In addition, while in Alton et al. (1998) the $200 \mu \mathrm{m}$ scale-length is $80 \%$ longer than at $60 \mu \mathrm{m}$ (with a mean ratio of $\sim 1.8$ ), we find that the 160 and $250 \mu \mathrm{m}$ scale-lengths are 10 and $30 \%$ larger, respectively, than that at $70 \mu \mathrm{m}\left(\left\langle h_{160 \mu \mathrm{m}} / h_{70 \mu \mathrm{m}}\right\rangle \sim 1.1,\left\langle h_{250 \mu \mathrm{m}} / h_{70 \mu \mathrm{m}}\right\rangle \sim 1.3\right)$.

As highlighted in the previous section, the dust-mass surface density we obtained through SED fitting to the Herschel data has an average scale-length that is $\sim 1.8$ times the stellar one. This dust-mass/stars scale-length ratio is slightly higher than those found by Xilouris et al. $(1999 ; 1.4 \pm 0.2)$ and Bianchi $(2007 ; 1.5 \pm 0.5)$ with radiative transfer fits of the dust extinction 
lanes in edge-on spirals, and compatible with that derived by De Geyter et al. (2014; $1.8 \pm 0.8$, also in this case by using radiative transfer fits).

Muñoz-Mateos et al. (2009a) derived the dust surfacedensity profiles of SINGS galaxies by fitting Spitzer SED profiles with the Draine \& Li (2007) method. After fitting the profile with an exponential curve, they found that the dust scale-lengths in the sample are 1.1 the stellar scale-lengths derived from $3.6 \mu \mathrm{m}$ data ${ }^{14}$. From the median value of the $r_{25}$-normalized dust scale-length (0.29) one can obtain the $r_{25}$-normalized $3.6 \mu \mathrm{m}$ scale-length (0.26), which is in excellent agreement with what we derived here with our smaller sample $\left(\left\langle h_{3.6 \mu \mathrm{m}} / r_{25}\right\rangle \sim 0.24\right)$. Instead, the dust surface-density scale-length is smaller than what is inferred from fits to edge-on galaxies and almost half of what we derive here from fitting the surface-density maps. However, this does not necessarily indicate a disagreement between our work and that of Muñoz-Mateos et al. (2009a). As recognized by the same authors, this is most likely the result of the longest wavelength available in their SED, the $160 \mu \mathrm{m}$ data from Spitzer; without a full coverage of the thermal emission peak and beyond, they could not probe the full temperature gradient with the emission from colder dust, and thus their dust-mass scalelengths might be simply a reflection of the surface-brightness scale-lengths at $160 \mu \mathrm{m}$.

Exponential scale-lengths of the surface-brightness profiles at 3.6 and $250 \mu \mathrm{m}$ for the KINGFISH sample have been determined by Hunt et al. (2015). At $250 \mu \mathrm{m}$, they found $h_{250 \mu \mathrm{m}} / r_{25}=0.35$, a value larger than our determination and that of Muñoz-Mateos et al. (2009a). The main reason for the difference seems to be the radial range used by Hunt et al. (2015); to avoid any contribution from the bulge, even in early type spirals, they fitted data for $r / r_{\text {opt }} \geq 0.6$ and up to $r / r_{\text {opt }}=1.5$. This range has a small overlap with that used in our analysis, which refers to the inner disks and whose choice was dictated by the necessity of a uniform coverage for all the wavelengths we considered. In fact, for the 12 galaxies we have in common with the KINGFISH sample (which are essentially from the same Herschel and Spitzer datasets), we find that the profiles of Hunt et al. (2015) are similar to our own for the inner disks, and tend to flatten in the range used in their work.

The methodology in the analysis of Hunt et al. (2015) might also be responsible for the larger estimate of the scalelength at $3.6 \mu \mathrm{m}$ : they find $h_{3.6 \mu \mathrm{m}} / r_{25}=0.37$, a value which, similarly to that at $250 \mu \mathrm{m}$, is larger than those obtained for stars by other authors (e.g., Giovanelli et al. 1994; Moriondo et al. 1998; Hunt et al. 2004) and on SINGS galaxies by Muñoz-Mateos et al. (2009a). Part of the differences might be due to the method of sky subtraction in Hunt et al. (2015). Nevertheless, the authors claim that the homogeneity in the image and profile extraction processing at different wavelengths should obviate possible biases that could affect their conclusions (as well as ours and those of Muñoz-Mateos et al. 2009a), in particular on scale-length ratios. Indeed, Hunt et al. (2015) find a mean ratio between the $250 \mu \mathrm{m}$ scale-length and the $3.6 \mu \mathrm{m}$ one of about 1 , the same value we derive here. We cannot extend our comparison to other tracers of cold dust emission, since exponential fits at longer sub-mm wavelengths are not available in Hunt et al. (2015).

\footnotetext{
14 The common resolution of the analysis of Muñoz-Mateos et al. (2009a) at 3.6 and $160 \mu \mathrm{m}$ and of Hunt et al. (2015) at 3.6 and $250 \mu \mathrm{m}$ is that of the Spitzer/MIPS instrument at $160 \mu \mathrm{m}$ maps $\left(F W H M=38^{\prime \prime}\right)$, very close to that of Herschel/SPIRE at $500 \mu \mathrm{m}$ maps, adopted here.
}

More complex is the comparison with the recent analysis of Smith et al. (2016). They stacked azimuthally averaged profiles for a large sample of low-inclination HRS galaxies and detect dust emission up to a distance of $2 \times r_{25}$. From the stacked Herschel profile of the largest 45 objects in their sample, they derived the radial variation of the dust SED, and a dust-mass surface-density after fitting a MBB to the SED in each radial bin. Though results are provided for fits up to $2 \times r_{25}$, the exponential behavior of their profiles within the inner $r_{25}$ is not much different from the full one and thus could be compared with ours. For the common resolution of $500 \mu \mathrm{m}$ data, they provide gradients for the dust-mass surface density, the stellar-mass surface density and the SFR, normalized to $r_{25}$. From their gradients, we derived a $r_{25}$-normalized scale-length for the dust-mass surface density of 0.25 . Not only do we find a larger dust mass $r_{25}$-normalized scale-length (0.42) but also a larger ratio between the dust and $500 \mu \mathrm{m}$ scale-lengths (1.33), while the $500 \mu \mathrm{m}$ stacked profile presented in their Fig. 2 has almost the same scale-length as the dust mass.

In principle, the result of Smith et al. (2016) could be explained with a much shallower temperature gradient. Indeed, the sub-mm profiles shown in their Fig. 2 give a slightly smaller $h_{500 \mu \mathrm{m}} / h_{250 \mu \mathrm{m}}$ ratio (1.15 vs. 1.25 , determined from their deconvolved exponential fits for the same radial range used in the current work). However, the scale-length of the average temperature gradient for $r / r_{25}<1$, derived from their Fig. 4, is $h_{\mathrm{T}} / r_{25} \approx 1.8$, which, assuming $U \approx T^{4+\beta}$ (Hunt et al. 2015, they used $\beta=2$ ), converts to $h_{U} / r_{25} \approx 0.3$, again smaller than our determination. Thus, their dust heating gradient is steeper than ours, and should have produced a larger ratio for $h_{500 \mu \mathrm{m}} / h_{250 \mu \mathrm{m}}$ and a larger $h_{\Sigma_{\text {dust }}} / h_{500 \mu \mathrm{m}}$ than in this work, which is contrary to what they derive. Clearly, the complex procedure adopted for the stacked analysis makes it complicated to understand the reason for this discrepancy. We only note that we get discrepant results also for the SFR surface density scale-length, which in our case has a value in between those of the UV and NIR surfacebrightness distributions used to derive it $(0.24)$; while in theirs it is smaller (0.16) than in the NIR (our determination), despite the similarity of the methods used to derive it. Instead, their stellarmass surface-density has a scale-length similar to our own (they have 0.24 ). Thus, they obtain a dust to stellar mass scale-length ratio of $\sim 1$, in contrast with our previous discussion.

The trend of increasing $70 \mu \mathrm{m}$ scale-length with respect to $3.6 \mu \mathrm{m}$ and $250 \mu \mathrm{m}$ emission for increasing Hubble stage $T$ can instead be the result of a residual bulge contribution which has escaped our simple 1D analysis and masking of the inner regions. A more concentrated heating source could result in a steeper gradient to dust heating for earlier-type galaxies, as seen in the models of Bianchi (2008). The weak increasing trend of the $h_{\Sigma \mathrm{SFR}} / h_{3.6 \mu \mathrm{m}}$ ratio moving to the later types is expected in the framework's inside-out scenario of galaxy disks. As shown in the grid of chemical evolution models of Mollá \& Díaz (2005) (e.g., their Fig. 7, panel b), at a given galaxy mass, galaxies with different SF efficiencies (that can be translated into different morphological types) result in different SF histories. Spiral galaxies of later type, thus having lower SF efficiency, tend to have very low SFR in the past and to have the peak of their SFR at present time, while early type spiral (with higher SF efficiencies) had this peak a few Gyr after their formation. At later times, the SFR of early type spirals is maintained constant. Thus, we expect differences in the scale-lengths of galaxies of different morphological type due to their different SF histories. In particular, we expect that in the late type galaxies, the SFR might be more spatially extended at present time than in the past because they have just reached or 
they are still reaching the peak of their SFR. On the other hand, earlier-type galaxies are in stationary phase after the peak of the SFR. Consequently the comparison of the scale-lengths are essentially unchanged with time.

The dependence on the wavelength flattens for the MIR dust emission bands, because of a less strong dependence (a linear one) on the interstellar radiation field of dust emission from very small grains subject to stochastic heating (Draine \& Li 2007). At $12 \mu \mathrm{m}$, we find an average scale-length with respect to $3.6 \mu \mathrm{m}$ emission, $\left\langle h_{12 \mu \mathrm{m}} / h_{3.6 \mu \mathrm{m}}\right\rangle$, of 0.9 which is close to what has been derived at $8 \mu \mathrm{m}$, another band dominated by emission features, by Regan et al. (2006; 0.97, as derived from their tabulated data).

The large scale-lengths found in this work for the dustmass surface brightness imply, if there is no disk truncation, that $\approx 30 \%$ of the dust mass resides in the outskirts of the disk at $r>r_{25}$, as opposed to only $\approx 10 \%$ for the stellar mass. Together with the previous results from radiative transfer fits of edge-on galaxies, this poses the problem of how dust can migrate from its origin in evolved stellar atmospheres and $\mathrm{SNe}$, or, as we found here when comparing dust and molecular gas, from the denser parts of the ISM where it is supposed to accrete most of its mass. Several qualitative explanations for the large dust scale-lengths have been proposed: the dust could trace an "unseen" molecular gas component at large radii, or grains could be transported from the optical disk, through the action of macro turbulence, or thanks to interactions with nearby galaxies (for a review, we refer to Sauvage et al. 2005). For the well-studied case of the edge-on galaxy NGC 891, the dust emission detected at $r \approx r_{25}$ (Popescu \& Tuffs 2003) is likely associated to nonpristine gas from the inner disk perturbed by the nearby companion UGC 1807 (Bianchi \& Xilouris 2011); however, the detected feature constitutes only a minor fraction of the total dust mass of the galaxy.

Despite the fact that the galaxies of our sample are classified as interacting (see Sect. 2), we cannot invoke a similar mechanism to explain our results; our dust mass scale-lengths, as well as all the other measurements, refer only to the inner disk for $r<r_{25}$ and there is no indication (but also no proof of the contrary) that the observed behavior extends beyond the optical disk. Despite the increased sensitivity of the Herschel telescope and other instruments, the dust mass fraction beyond $r_{25}$ cannot be easily derived for individual galaxies; instead, stacking analysis seems to suggest that stars and dust follow the same distribution up to $2 \times r_{25}$ (Smith et al. 2016, though this result, as we discussed earlier, is not consistent with that of this work for $r<r_{25}$ ). A possible mechanism that could explain our findings is a change in the typical lifetimes of grains against destruction by shocks, with a longer lifetime at larger radii (Sauvage et al. 2005). Because of the similarity between UV and dust scale-lengths, it is tempting to associate this mechanism to the inside-out galaxy formation scenario that is used to explain, together with dust extinction, the larger scale-lengths of radiation from the younger stellar component (Muñoz-Mateos et al. 2009a). The planned radiative transfer analysis of DustPedia galaxies will shed light on the true contribution of dust extinction to the shaping of the UV and optical appearance of spiral galaxies, and provide a detailed description of the disk surface brightness across the SED of dust emission. This will allow us to analyze in detail why some galaxies present variations to common trends of scale-length versus wavelength (e.g., NGC 5457 (M 101), NGC 5194 (M 51), and NGC 6946).

As for the molecular gas, its scale-length is, on average, smaller than that of the optical-NIR bands $\left(\left\langle h_{\Sigma \mathrm{H} 2} / h_{3.6 \mu \mathrm{m}}\right\rangle \sim 0.7\right.$, $\left.\left\langle h_{\Sigma \mathrm{H} 2} / h_{12 \mu \mathrm{m}}\right\rangle \sim 0.8\right)$. Leroy et al. (2009) found that the average molecular gas scale-length is only $5 \%$ smaller than the $3.6 \mu \mathrm{m}$ stellar scale-length and the SFR scale-length, while Regan et al. (2001, 2006) and Bendo et al. (2010) find that molecular gas and optical-NIR bands have profiles with comparable scalelengths. The comparison between the scale-lengths of molecular gas and MIR emission features could be conditioned by using different ${ }^{12} \mathrm{CO}$ transitions and MIR bands. For the molecular gas, we used ${ }^{12} \mathrm{CO}(1-0)$ and ${ }^{12} \mathrm{CO}(2-1)$ emission lines, while Regan et al. (2006) used the ${ }^{12} \mathrm{CO}(1-0)$ emission line, and Bendo et al. (2010) the ${ }^{12} \mathrm{CO}(3-2)$ emission line. Further, for the MIR emission features, we analyzed the images at $12 \mu \mathrm{m}$, while Regan et al. (2006) and Bendo et al. (2010) analzyed those at $8 \mu \mathrm{m}$. It is well known that each ${ }^{12} \mathrm{CO}$ transition traces different physical gas properties. The kinematic temperature of molecular gas is typically $\sim 10 \mathrm{~K}$ (Scoville et al. 1987), which is above the level energy temperature of $5.5 \mathrm{~K}$ for the $J=1$ level of the ${ }^{12} \mathrm{CO}$, but below the temperatures of $16.5 \mathrm{~K}$ and $33 \mathrm{~K}$ for the $J=2$ and $J=3$ levels, respectively. This implies that a slight change in gas kinematic temperature is sufficient to affect the excitation for the ${ }^{12} \mathrm{CO}(2-1)$ and ${ }^{12} \mathrm{CO}(3-2)$ emission lines. In addition, the different critical densities of the three ${ }^{12} \mathrm{CO}$ transitions $\left(\sim 10^{3} \mathrm{~cm}^{-3}\right.$, $\sim 2 \times 10^{4} \mathrm{~cm}^{-3}$, and $\sim 7 \times 10^{4} \mathrm{~cm}^{-3}$ for ${ }^{12} \mathrm{CO}(1-0),{ }^{12} \mathrm{CO}(2-1)$, and ${ }^{12} \mathrm{CO}(3-2)$, respectively) make their line ratios sensitive to local gas density. The emissions at 8 and $12 \mu \mathrm{m}$ are instead attributable to the same kind of transitions in $\mathrm{C}-\mathrm{H}$ bonds in the proposed carriers of the MIR features, like, for example, PAH molecules (Draine 2011) or nanoparticles of amorphous hydrocarbons (Jones et al. 2013). The main result of the current analysis being that the dust-mass surface-density scale-length is about 1.8 times the stellar one, a significative fraction of the refractory materials must reside in the atomic gas. Based on these results, in the following section we attempt to derive the relative contribution of $\mathrm{H}_{2}$ and $\mathrm{HI}$ to the ISM, and the $\alpha_{\mathrm{CO}}$ conversion factor.

\section{Dust- and gas-mass profiles to solve for single CO-to- $\mathrm{H}_{2}$ conversion factors}

As already mentioned in Sect. 5.3, a major uncertainty in the derivation of the molecular gas content in a galaxy is the poorlyknown $\alpha_{\mathrm{CO}}$ conversion factor. We have developed a method to solve for the $\alpha_{\mathrm{CO}}$ conversion factor per galaxy by using dustand gas-mass profiles.

Recently, for resolved galaxies with available Herschel data, it has been shown that it is possible to derive $\alpha_{\mathrm{CO}}$ independently of the dust-mass estimate and DGR (Sandstrom et al. 2013). The idea of Sandstrom et al. (2013) is that spatially resolved measurements of $\Sigma_{\text {dust }}, \Sigma_{\mathrm{HI}}$, and $\Sigma_{\mathrm{H} 2}$ (from CO observations) allow one to solve for $\alpha_{\mathrm{CO}}$ and the DGR over regions smaller than the typical scale over which the DGR varies (i.e., one region is well represented by a single DGR value) and it covers a range of $\mathrm{CO} / \mathrm{HI}$ ratios. The peculiarity of their approach is the absence of assumptions about the values of $\alpha_{\mathrm{CO}}$ and the DGR and their dependence on metallicity or other parameters. Another approach has been developed by Magrini et al. (2011). Using typical properties of Galactic dust and gas, they converted the DGR profile into a profile of the oxygen abundances for the metals in solid state; they then compared it to the gas metallicity gradients and studied the dependency of the $\alpha_{\mathrm{CO}}$ conversion factor on metallicity.

The method we developed to solve for the $\alpha_{\mathrm{CO}}$ conversion factor per galaxy is a simplified version of that of Magrini et al. (2011) and is based on the following assumptions: i) The DGR and the dust optical properties are the same in both the atomic and molecular gas phases; ii) dust and gas are well mixed; iii) the 
DGR is invariant with respect to the metallicity gradient or it follows the same trend of the metallicity gradient; and iv) the fraction of mass in ionized gas is negligible.

As early as the mid-1980s, Hildebrand (1983) suggested that a good way to estimate the mass and the distribution of the ISM in a galaxy might be from the optical depth of the sub$\mathrm{mm}$ continuum emission from the dust. In later years, experimental studies based on laboratory samples of dust grain species have instead shown that there is less scatter between species around $100 \mu \mathrm{m}$ than in the sub-mm (e.g., Coupeaud et al. 2011). Dust grains are robust and found in all phases of the ISM. Several works therefore used the dust emission to infer the gas distribution (e.g., Guelin et al. 1993, 1995; Boselli et al. 2002; Corbelli et al. 2012; Eales et al. 2012; Sandstrom et al. 2013; Groves et al. 2015). The amount of gas is usually measured using HI and CO surveys, however Smith et al. (2012) have found that for early-type galaxies (E/S0) the ISM was detected for $50 \%$ of objects through its dust emission but only $22 \%$ through its CO emission, in addition to the uncertainty associated with $\alpha_{\mathrm{CO}}$.

From a practical point of view, in order to solve for the $\alpha_{\mathrm{CO}}$ conversion factor per galaxy, we first determined the dust-, atomic-, molecular- and total-gas-mass profiles following prescriptions of Sect. 5, in particular by assuming the constant $\alpha_{\mathrm{CO}}$ value of $3.2 M_{\odot} \mathrm{pc}^{-2}\left(\mathrm{~K} \mathrm{~km} \mathrm{~s}^{-1}\right)^{-1}$. Second, based on our assumptions and in the hypothesis of a constant global value for the DGR, we can write:

$$
\begin{aligned}
\Sigma_{\text {dust }} & =\operatorname{DGR}\left(\Sigma_{\mathrm{HI}}+\Sigma_{\mathrm{H} 2}\right) \\
& =\operatorname{DGR}\left(\Sigma_{\mathrm{HI}}+\alpha_{\mathrm{CO}} I_{\mathrm{CO}}\right) .
\end{aligned}
$$

Finally, we fitted Eq. (7) to the data and derived global values for DGR and $\alpha_{\text {CO }}$ for each galaxy.

We applied the same approach described above, also taking into account that the DGR is an indicator of the amount of metals that get locked up in the dust through the stellar yields. Thus, a dependence on the metallicity gradient is expected. A correlation has been widely shown between the DGR and the gas-phase oxygen abundance (e.g., Issa et al. 1990; Lisenfeld \& Ferrara 1998; Edmunds 2001; James et al. 2002; Hirashita et al. 2002; Draine et al. 2007): the DGR decreases with radius, following the trend with metallicity. We therefore corrected atomic- and molecular-gas-mass profiles for metallicity thanks to the radial metallicity gradient available for the entire galaxy sample (see Sect. 3.7). To do this, we applied Eq. (7) with the following additional assumption:

$\log (\mathrm{DGR})=\log \left(\mathrm{DGR}_{0}\right)+C_{\mathrm{O} / \mathrm{H}} \times\left(r / r_{25}\right)$,

(i.e., the same gradient as for metallicity given by Eq. (1)) with $\mathrm{DGR}_{0}$ being the value at $r_{0}=0$. Thus, we also derived global values for DGR and $\alpha_{\mathrm{CO}}$ "corrected" for metallicity gradient $\left(\alpha_{\mathrm{CO}(\mathrm{O} / \mathrm{H})}\right)$. Again, we do not comment on the $\mathrm{DGR}_{0}$ values.

The right panels of Figs. 3 and A.2 show the mass-surfacedensity profiles of the dust, atomic, molecular, and total gas corrected using the derived DGR and $\alpha_{\mathrm{CO}}$ according to Eq. (7) without the correction for metallicity gradient of Eq. (8). In the case of one available gas component ( $\mathrm{HI}$ or $\mathrm{H}_{2}$ ), we corrected it by using the derived DGR. Given the uncertainties in the dust-mass derivation and since this work is focused on the study of the gradients, we do not attach any particular importance to the values of DGR. We use these values to scale the profiles in Figs. 3 and A.2. The estimated $\alpha_{\mathrm{CO}}$ values are instead more directly related to the relative gradients of the various ISM components.

The $\alpha_{\mathrm{CO}}$ values derived without or with the correction for metallicity gradient are collected in Table 8. In this table, Col. (1) gives the galaxy names, Col. (2) the metallicity gradients with uncertainty from Pilyugin et al. (2014), Col. (3) the oxygen abundances at the radius of $0.7 \times r_{25}$ that is equivalent to the solar radius in our Galaxy, Cols. (4) and (5) $\alpha_{\mathrm{CO}}$ values derived without and with the correction for metallicity gradient, respectively, and Cols. (6) and (7) mean $\alpha_{\mathrm{CO}}$ values derived from Sandstrom et al. (2013) for the galaxies in common with our sample for all galaxies and the inner galactic kpc, respectively.

The $\alpha_{\mathrm{CO}}$ values are in the range (0.39) $M_{\odot} \mathrm{pc}^{-2}\left(\mathrm{~K} \mathrm{~km} \mathrm{~s}^{-1}\right)^{-1}$ both without and with the correction of $\mathrm{HI}$ and $\mathrm{H}_{2}$ profiles for metallicity. The $\alpha_{\mathrm{CO}}$ determinations obtained taking into account the metallicity gradient are always lower than (or equal to) those derived neglecting the metallicity gradient. The highest discrepancy between the two $\alpha_{\mathrm{CO}}$ derivations $\left(\alpha_{\mathrm{CO}} / \alpha_{\mathrm{CO}(\mathrm{O} / \mathrm{H})} \sim 1.9\right)$ is found for NGC 3521, the galaxy with the second-steepest metallicity gradient in our sample, $\sim 0.7$ dex $r_{25}{ }^{-1}$. This could be due to the very small number of oxygen abundances (9) used to derive the metallicity gradient for NGC 3521 (we refer to Fig. 1 of Pilyugin et al. 2014), giving statistically weak metallicity gradients with respect to those of other galaxies (e.g., NGC 628 and NGC 5457 (M 101) where the metallicity gradients are derived from several tens of oxygen abundances). The salient point is that the correction of gas profiles for metallicity gradient has no significant influence on the $\alpha_{\mathrm{CO}}$ value at least for the present galaxy sample. This happens although $\mathrm{O} / \mathrm{H}$ gradients of our sample range from -0.066 dex $r_{25}$ (almost flat) to $-0.840 \mathrm{dex} r_{25}$ (very steep, we refer to e.g., Magrini et al. 2016, for examples of flat and steep metallicity gradients). Because of this, we did not explore the possibility of a further dependence of the $\alpha_{\mathrm{CO}}$ value on the metallicity gradient.

The almost invariance of the $\alpha_{\mathrm{CO}}$ value by adopting a fixed DGR or a DGR depending on the metallicity gradient can mean that $\alpha_{\mathrm{CO}}$ is mainly constrained by the central DGR value in the galaxy or that the DGR values derived from both approaches are different to give the same resulting $\alpha_{\mathrm{CO}}$. For our sample the explanation could be a compromise of both causes: in the central regions dominated by molecular gas, the DGR values are almost independent of the metallicity gradient, while in outer parts rich in atomic gas, the DGR values seem to be metallicitygradient-dependent. The final result is that $\alpha_{\mathrm{CO}}$ is independent of the metallicity gradient for our sample.

\subsection{CO-to- $\mathrm{H}_{2}$ values: comparison with the literature}

Many authors have derived $\alpha_{\mathrm{CO}}$ using a variety of techniques: measuring total gas masses from $\gamma$-ray emission plus a model for the cosmic ray distribution (e.g., Strong \& Mattox 1996; Abdo et al. 2010); using the observed velocity dispersion and size of the molecular cloud to obtain virial masses (e.g., Solomon et al. 1987; Wilson 1995; Bolatto et al. 2008; Gratier et al. 2012); and modeling multiple molecular gas lines with varying optical depths and critical densities (e.g., Weiß et al. 2001; Israel 2009a,b). We do not summarize results of the literature, but we focus on the comparison between our derivations of $\alpha_{\mathrm{CO}}$ and those of Sandstrom et al. (2013) who solved for $\alpha_{\mathrm{CO}}$ with a method similar to ours and for eight galaxies present in our sample.

In four (NGC 5457 (M101), NGC 3521, NGC 925, NGC 628 (M74)) of the eight galaxies in common with Sandstrom et al. (2013), our $\alpha_{\mathrm{CO}}$ derivations referring to the galactic region within $r_{25}$ (or minor if gas-mass profiles are not available until to $r_{25}$ ) are similar to (for 3 or 4 galaxies consistent with) their values within $r_{25}$ (we refer to Col. (6) of Table 8). For 
A\&A 605, A18 (2017)

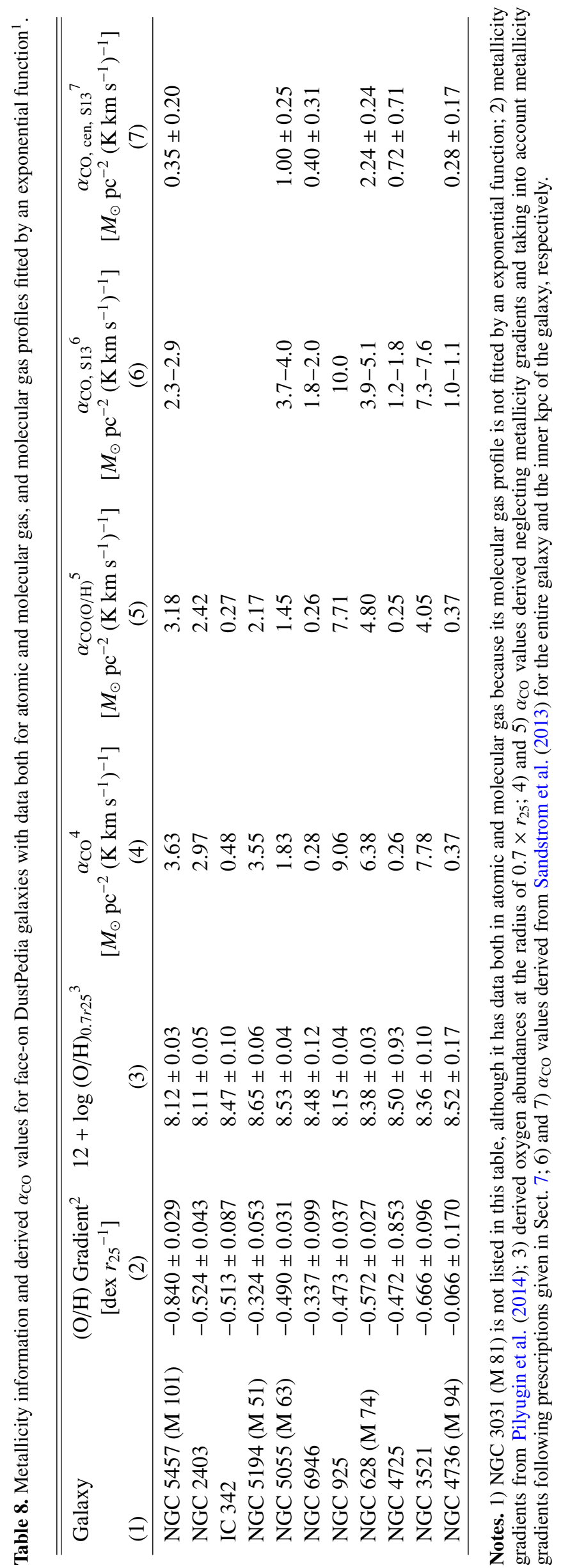


the other four galaxies (NGC 5055 (M63), NGC 4736 (M94), NGC 6946, NGC 4725), there is instead an evident discrepancy between the two $\alpha_{\mathrm{CO}}$ determinations, with our values being lower than those of Sandstrom et al. (2013) within $r_{25}$ but more similar to their determinations for galaxy centers (we refer to Col. (7) of Table 8). Sandstrom et al. (2013) indeed found relatively flat $\alpha_{\mathrm{CO}}$ profiles as a function of galactocentric radius apart from in the galaxy centers (within the inner $\sim 0.1 \times r_{25}$ ) that have $\alpha_{\mathrm{CO}}$ values lower than the galaxy mean ones by factors of 2-3 (or more).

The reason of the discrepancy for four galaxies in common with the sample of Sandstrom et al. (2013), is difficult to establish, but is likely due to differences between the two methods for deriving $\alpha_{\mathrm{CO}}$ (e.g., the work of Sandstrom et al. 2013, is based on circular regions while we used radially averaged profiles), and to how different methods correlate with the ISM properties of individual galaxies; local environmental conditions (metallicity, galactic dynamics, ISM pressure, interstellar radiation field strength, optical depth of the ISM, gas temperature, and dust properties) can produce variations in $\alpha_{\mathrm{CO}}$ (Sandstrom et al. 2013). Additionally, our sample contains some galaxies defined as SF or HII region-dominated (e.g., NGC 5457 (M 101), NGC 6946), and others with signatures of AGN or LINER activity (e.g., NGC 4736 (M 94)).

\subsection{Caveats about the derivation of $\mathrm{CO}-$ to- $\mathrm{H}_{2}$ conversion factor}

The method we developed to derive $\alpha_{\mathrm{CO}}$ could not fit the dust and total gas profiles for the entire galaxy sample (we refer to Figs. 3 and A.2 for the shape of dust and gas profiles of the single galaxies). The assumption that the DGR is not a function of atomic/molecular phase could, for instance, be the cause of the low $\alpha_{\text {CO }}$ values derived for NGC 4736 (M 94) and NGC 6946. In NGC 4736 (M 94), the HI gas profile has indeed a similar behavior to the $\mathrm{H}_{2}$ one, with a central peak and a strong decrease towards the outer parts, while the HI gas distribution is typically low in the inner parts and it increases at large radius. In NGC 6946, the gas profiles show expected trends but the $\mathrm{H}_{2}$ one is strongly peaked in the center with respect to the increase of the HI distribution in the outer parts. Implicit with our assumption on the DGR is the assumption that the dust properties do not change significantly across the different environments that are best sampled by the Herschel resolution. In the current method, and in the analogous works from the literature that we have referred to, the dust mass is derived using the same optical properties, those of the diffuse medium, regardless of the (unknown) contribution of dust associated to molecular- or atomic gas in a specific position within a galaxy. The assumption could be valid if the evolution of dust in denser environments (and in particular the changes in optical properties of grains resulting from coagulation and accretion; we refer to, e.g., Köhler et al. 2015) are restricted to regions with a small filling factor with respect to the global distribution of molecular gas. On this assumption relies not only the entire analysis of this paper, as stated in Sect. 5.1, but also the many dust-mass determinations in the literature: only detailed modeling of the various phases of the ISM in a galaxy, of the optical properties of dust in each phase, and of the local heating conditions will help in the future to understand if the use of a single diffuse-medium dust model still provide a reasonable estimate, or instead introduce a strong bias in the determination of the dust-mass surface density or global values.

The galaxy NGC 628 (M 74) gives the best results of our method. First, its scale-length ratio $h_{\Sigma \text { tot gas }} / h_{\Sigma \text { dust }}$ is around unity and this means that the assumption that the total gas profile follows the dust one is reasonable for this galaxy. Secondly, dust and gas profiles are available for the same radius range. Under these conditions the method we developed gives a $\alpha_{\mathrm{CO}}$ consistent with determinations from other authors (e.g., Sandstrom et al. 2013).

The scale-length ratio $\left\langle h_{\Sigma \text { tot gas }} / h_{\Sigma \text { dust }}\right\rangle$ averaged over the galaxy sample is $\sim 1.1$, and the excess, although small, with respect to the unity could suggest that dust and gas are not so well mixed for all galaxies, contrary to what we assumed in our method to derive $\alpha_{\mathrm{CO}}$. This should simply mean that the DGR varies non-linearly with metallicity, as in the outskirts $\mathrm{H}_{2}$ is negligible. For a large (126) sample of local Universe galaxies covering a 2 dex metallicity range, Rémy-Ruyer et al. (2014) found that the chemical evolution of galaxies (especially for lowmetallicity galaxies), traced by their DGR, strongly depends on their local internal conditions and individual histories. Finally, the scale-length ratio $\left\langle h_{\Sigma \text { tot gas }} / h_{\Sigma \text { dust }}\right\rangle$ larger than 1 could also be due to the fact that for some galaxies the $\mathrm{H}_{2}$ gas profile is available for a smaller radius with respect to the dust and $\mathrm{HI}$ profiles (e.g., NGC 5055 (M 63), NGC 2403).

\section{Conclusions}

We studied the radial distribution of dust, stars, gas, and SFR in a sub-sample of 18 spiral face-on galaxies extracted from the DustPedia sample. The selection was based on galaxies imaged over their whole extent with both PACS and SPIRE in Herschel, with $1 \leq T \leq 8,(d / D)_{\text {sub-mm }} \geq 0.4$, and $D_{\text {sub-mm }} \geq 9^{\prime}$. This study exploited the multi-wavelength (UV/sub-mm) DustPedia database, together with millimeter and centimeter maps extracted from other sources and information on metallicity abundances available in the literature. We fitted the surface-brightness profiles of the tracers of dust and stars, the mass surface-density profiles of dust, stars, molecular gas, and total gas, and the SFR surface density with a simple exponential curve and derived their exponential scale-lengths.

We identified average trends of the scale-lengths with wavelengths: the scale-length decreases from UV to $70 \mu \mathrm{m}$, and this effect can be due to an intrinsic gradient in the stellar populations with a blueing galaxy disk at larger distance from the center, to a preferential reddening in the inner regions of a galaxy, or to the combined role of dust and stars in shaping the gradient. The scale-length increases again from 70 to $500 \mu \mathrm{m}$, most likely because of the cold-dust temperature gradient with galactocentric distance. The scale-lengths of the dust-mass surface density are larger than those of the surface-brightness profiles at $500 \mu \mathrm{m}$, generally larger than any of the other surface-brightness scale-lengths and than the stellar-mass surface-density distributions. On average, the dust-surface-density scale-length is about 1.8 times the stellar density one derived from IRAC data or the $3.6 \mu \mathrm{m}$ surface-brightness scale-length, a result in agreement with radiative transfer analysis of dust extinction in edgeon galaxies. This result could be explained by a change in the typical lifetimes of grains against destruction by shocks, with a longer lifetime at larger radii. We also found that UV and dust scale-lengths are similar and this could be associated with the inside-out galaxy formation scenario that is used to explain, together with dust extinction, the larger scale-lengths of radiation from the younger stellar component. As for the gas, the $\mathrm{H}_{2}$ surface-density profiles have typically scale-lengths $\sim 20 \%$ smaller than the NIR surface brightness, and - on average a factor 2.3 smaller than the dust ones. The total gas surface 
density has $\sim 10 \%$ flatter profiles than dust, though the result is influenced by the $\alpha_{\mathrm{CO}}$ value.

By studying the trends of the scale-lengths as a function of Hubble stage $T$, we found that the ratio between the scalelengths of the Herschel bands and the $3.6 \mu \mathrm{m}$ scale-length tends to increase from earlier to later types, and this trend is particularly evident for the ratio $h_{70 \mu \mathrm{m}} / h_{3.6 \mu \mathrm{m}}$. The value for $h_{70 \mu \mathrm{m}}$ instead tends to be smaller than that at longer sub-mm wavelengths with ratios between longer sub-mm wavelengths and $70 \mu \mathrm{m}$ that decrease with increasing $T$ (see $h_{250 \mu \mathrm{m}} / h_{70 \mu \mathrm{m}}$ ). These trends could be the result of a bulge contribution - that escaped our simple 1D analysis - to dust heating for earlier-type galaxies. Among the surface-density ratios, only $h_{\Sigma \mathrm{SFR}} / h_{3.6 \mu \mathrm{m}}$ shows a weak trend of increasing SFR scale-length, with respect to stars, towards later types, compatible with longer infall time scales for late-type galaxies.

We developed a method to solve for single $\alpha_{\mathrm{CO}}$ conversion factor for each sample galaxy with both atomic and molecular gas data, by assuming that the total gas profile mimics the dust one. For the derivation of $\alpha_{\mathrm{CO}}$, we also took into account the dependence of DGR on metallicity. We found values of $\alpha_{\mathrm{CO}}$ in the range (0.3-9) $M_{\odot} \mathrm{pc}^{-2}\left(\mathrm{~K} \mathrm{~km} \mathrm{~s}^{-1}\right)^{-1}$ both with and without the correction of $\mathrm{HI}$ and $\mathrm{H}_{2}$ profiles for metallicity. The determinations of $\alpha_{\mathrm{CO}}$ obtained taking into account the metallicity gradient are always lower than (or equal to) those derived neglecting the metallicity gradient. In any case, the correction of gas profiles for metallicity gradient has no significant influence on the $\alpha_{\mathrm{CO}}$ value, at least for our galaxy sample with mean metallicity ranging from $12+\log (\mathrm{O} / \mathrm{H}) \sim 8.3$ to $12+\log (\mathrm{O} / \mathrm{H}) \sim 8.7$.

As an extension of this work, in our following papers in the frame of the DustPedia project, we are about to compare single Sérsic decomposition parameters retrieved for all DustPedia sample galaxies for the WISE W1 and Herschel observations. Thus, we will be able to directly compare effective radii of the stellar population and dust.

Acknowledgements. We thank an anonymous referee for useful comments and suggestions, which improved the quality of the manuscript. DustPedia is a collaborative focused research project supported by the European Union under the Seventh Framework Programme (2007-2013) call (proposal No. 606824). The participating institutions are: Cardiff University, UK; National Observatory of Athens, Greece; Ghent University, Belgium; Université Paris Sud, France; National Institute for Astrophysics, Italy and CEA (Paris), France. J.F. acknowledges the financial support from UNAM-DGAPA-PAPIIT IA104015 grant, Mexico. This research made use of the NASA/IPAC Extragalactic Database (NED), which is operated by the Jet Propulsion Laboratory, California Institute of Technology, under contract with the National Aeronautics and Space Administration. We acknowledge the usage of the HyperLeda database (http: //leda.univ-lyon1.fr). This research has made use of the NASA/ IPAC In frared Science Archive, which is operated by the Jet Propulsion Laboratory, California Institute of Technology, under contract with the National Aeronautics and Space Administration. This publication makes use of data products from the Two Micron All Sky Survey, which is a joint project of the University of Massachusetts and the Infrared Processing and Analysis Center/California Institute of Technology, funded by the National Aeronautics and Space Administration and the National Science Foundation. This research made use of the VizieR catalogue access tool, CDS, Strasbourg, France.

\section{References}

Abdo, A. A., Ackermann, M., Ajello, M., et al. 2010, ApJ, 710, 810 Alton, P. B., Trewhella, M., Davies, J. I., et al. 1998, A\&A, 335, 807 Aniano, G., Draine, B. T., Gordon, K. D., \& Sandstrom, K. 2011, PASP, 123, 1218

Aniano, G., Draine, B. T., Calzetti, D., et al. 2012, ApJ, 756, 138

Asano, R. S., Takeuchi, T. T., Hirashita, H., \& Inoue, A. K. 2013, Earth Planets Space, 65, 213

Beckman, J. E., Peletier, R. F., Knapen, J. H., Corradi, R. L. M., \& Gentet, L. J. 1996, ApJ, 467, 175

Bell, E. F., \& de Jong, R. S. 2000, MNRAS, 312, 497
Bendo, G. J., Wilson, C. D., Warren, B. E., et al. 2010, MNRAS, 402, 1409 Bendo, G. J., Boselli, A., Dariush, A., et al. 2012, MNRAS, 419, 1833 Bendo, G. J., Baes, M., Bianchi, S., et al. 2015, MNRAS, 448, 135 Bianchi, S. 2007, A\&A, 471, 765

Bianchi, S. 2008, A\&A, 490, 461

Bianchi, S. 2013, A\&A, 552, A89

Bianchi, S., \& Xilouris, E. M. 2011, A\&A, 531, L11

Bigiel, F., Leroy, A., Walter, F., et al. 2008, AJ, 136, 2846

Bocchio, M., Marassi, S., Schneider, R., et al. 2016, A\&A, 587, A157

Bolatto, A. D., Leroy, A. K., Rosolowsky, E., Walter, F., \& Blitz, L. 2008, ApJ, 686, 948

Bolatto, A. D., Wolfire, M., \& Leroy, A. K. 2013, ARA\&A, 51, 207

Boquien, M., Calzetti, D., Combes, F., et al. 2011, AJ, 142, 111

Boselli, A., Lequeux, J., \& Gavazzi, G. 2002, A\&A, 384, 33

Boselli, A., Eales, S., Cortese, L., et al. 2010, PASP, 122, 261

Bramich, D. M., \& Freudling, W. 2012, MNRAS, 424, 1584

Bresolin, F., Kudritzki, R.-P., Urbaneja, M. A., et al. 2016, ApJ, 830, 64

Bruzual A., G., \& Charlot, S. 1993, ApJ, 405, 538

Bruzual, G., \& Charlot, S. 2003, MNRAS, 344, 1000

Buat, V. 1992, A\&A, 264, 444

Buat, V., \& Xu, C. 1996, A\&A, 306, 61

Buat, V., Deharveng, J. M., \& Donas, J. 1989, A\&A, 223, 42

Buat, V., Iglesias-Páramo, J., Seibert, M., et al. 2005, ApJ, 619, L51

Calzetti, D. 2001, PASP, 113, 1449

Calzetti, D., Kinney, A. L., \& Storchi-Bergmann, T. 1994, ApJ, 429, 582

Calzetti, D., Kennicutt, R. C., Jr., Bianchi, L., et al. 2005, ApJ, 633, 871

Calzetti, D., Kennicutt, R. C., Engelbracht, C. W., et al. 2007, ApJ, 666, 870

Camps, P., Trayford, J. W., Baes, M., et al. 2016, MNRAS, 462, 1057

Casasola, V., Bettoni, D., \& Galletta, G. 2004, A\&A, 422, 941

Casasola, V., Combes, F., Bettoni, D., \& Galletta, G. 2007, A\&A, 473, 771

Casasola, V., Combes, F., García-Burillo, S., et al. 2008, A\&A, 490, 61

Casasola, V., Hunt, L., Combes, F., \& García-Burillo, S. 2015, A\&A, 577, A135

Cassarà, L. P., Piovan, L., Weiss, A., Salaris, M., \& Chiosi, C. 2013, MNRAS, 436, 2824

Clark, C. J. R., Dunne, L., Gomez, H. L., et al. 2015, MNRAS, 452, 397

Clark, C. J. R., Verstocken, S., Fritz, J. et al. 2017, MNRAS, submitted

Compiègne, M., Verstraete, L., Jones, A., et al. 2011, A\&A, 525, A103

Corbelli, E., Bianchi, S., Cortese, L., et al. 2012, A\&A, 542, A32

Cortese, L. 2012, A\&A, 543, A132

Cortese, L., Boselli, A., Buat, V., et al. 2006, ApJ, 637, 242

Cortese, L., Ciesla, L., Boselli, A., et al. 2012, A\&A, 540, A52

Coupeaud, A., Demyk, K., Meny, C., et al. 2011, A\&A, 535, A124

Crosthwaite, L. P., Turner, J. L., \& Ho, P. T. P. 2000, AJ, 119, 1720

Crosthwaite, L. P., Turner, J. L., Hurt, R. L., et al. 2001, AJ, 122, 797

Cunow, B. 2004, MNRAS, 353, 477

Dale, D. A., Aniano, G., Engelbracht, C. W., et al. 2012, ApJ, 745, 95

Dale, D. A., Cook, D. O., Roussel, H., et al. 2017, ApJ, 837, 90

Davies, J. I., Alton, P., Bianchi, S., \& Trewhella, M. 1998, MNRAS, 300, 1006

Davies, J. I., Alton, P., Trewhella, M., Evans, R., \& Bianchi, S. 1999, MNRAS, 304, 495

Davies, J. I., Baes, M., Bianchi, S., et al. 2017, PASP, 129, 044102

de Blok, W. J. G., Walter, F., Brinks, E., et al. 2008, AJ, 136, 2648

De Geyter, G., Baes, M., Camps, P., et al. 2014, MNRAS, 441, 869

Deharveng, J.-M., Sasseen, T. P., Buat, V., et al. 1994, A\&A, 289, 715

de Jong, R. S. 1996, A\&A, 313, 377

De Vis, P., Dunne, L., Maddox, S., et al. 2017, MNRAS, 464, 4680

Draine, B. T. 2003, ARA\&A, 41, 241

Draine, B. T. 2009, Cosmic Dust - Near and Far, ASP Conf. Ser., 414, 453

Draine, B. T. 2011, Physics of the Interstellar and Intergalactic Medium (Princeton University Press)

Draine, B. T., \& Li, A. 2007, ApJ, 657, 810

Draine, B. T., Dale, D. A., Bendo, G., et al. 2007, ApJ, 663, 866

Dwek, E., Galliano, F., \& Jones, A. 2009, Cosmic Dust - Near and Far, ASP Conf. Ser., 414, 183

Eales, S., Smith, M. W. L., Auld, R., et al. 2012, ApJ, 761, 168

Eskew, M., Zaritsky, D., \& Meidt, S. 2012, AJ, 143, 139

Edmunds, M. G. 2001, MNRAS, 328, 223

Eufrasio, R. T., Dwek, E., Arendt, R. G., et al. 2014, ApJ, 795, 89

Fathi, K., Allen, M., Boch, T., Hatziminaoglou, E., \& Peletier, R. F. 2010, MNRAS, 406, 1595

Fazio, G. G., Hora, J. L., Allen, L. E., et al. 2004, ApJS, 154, 10

Ferrara, A., Viti, S., \& Ceccarelli, C. 2016, MNRAS, 463, L112

Ford, G. P., Gear, W. K., Smith, M. W. L., et al. 2013, ApJ, 769, 55

Galliano, F., Dwek, E., \& Chanial, P. 2008, ApJ, 672, 214

Gavazzi, G., \& Scodeggio, M. 1996, A\&A, 312, L29

Gavazzi, G., Pierini, D., \& Boselli, A. 1996, A\&A, 312, 397

Giovanelli, R., Haynes, M. P., Salzer, J. J., et al. 1994, AJ, 107, 2036

González-Martín, O., Masegosa, J., Márquez, I., et al. 2015, A\&A, 578, A74 
Gould, R. J., \& Salpeter, E. E. 1963, ApJ, 138, 393

Gratier, P., Braine, J., Rodriguez-Fernandez, N. J., et al. 2012, A\&A, 542, A108 Groves, B. A., Schinnerer, E., Leroy, A., et al. 2015, ApJ, 799, 96

Guelin, M., Zylka, R., Mezger, P. G., et al. 1993, A\&A, 279, L37

Guelin, M., Zylka, R., Mezger, P. G., Haslam, C. G. T., \& Kreysa, E. 1995, A\&A, 298, L29

Guilloteau, S., \& Lucas, R. 2000, Imaging at Radio through Submillimeter Wavelengths, ASP Conf. Proc., 217, 299

Hao, C.-N., Kennicutt, R. C., Johnson, B. D., et al. 2011, ApJ, 741, 124

Helfer, T. T., Thornley, M. D., Regan, M. W., et al. 2003, ApJS, 145, 259

Helou, G., Roussel, H., Appleton, P., et al. 2004, ApJS, 154, 253

Hildebrand, R. H. 1983, QJRAS, 24, 267

Hirashita, H., Tajiri, Y. Y., \& Kamaya, H. 2002, A\&A, 388, 439

Huang, M.-L., \& Kauffmann, G. 2015, MNRAS, 450, 1375

Hunt, L. K., Pierini, D., \& Giovanardi, C. 2004, A\&A, 414, 905

Hunt, L. K., Draine, B. T., Bianchi, S., et al. 2015, A\&A, 576, A33

Israel, F. P. 2009a, A\&A, 493, 525

Israel, F. P. 2009b, A\&A, 506, 689

Issa, M. R., MacLaren, I., \& Wolfendale, A. W. 1990, A\&A, 236, 237

James, A., Dunne, L., Eales, S., \& Edmunds, M. G. 2002, MNRAS, 335, 753

Jarrett, T. H., Masci, F., Tsai, C. W., et al. 2013, AJ, 145, 6

Jones, A. P., \& Nuth, J. A. 2011, A\&A, 530, A44

Jones, A. P., Fanciullo, L., Köhler, M., et al. 2013, A\&A, 558, A62

Jones, A. P., Koehler, M., Ysard, N., Bocchio, M., \& Verstraete, L. 2017, A\&A 602, A46

Jorsater, S., \& van Moorsel, G. A. 1995, AJ, 110, 2037

Kauffmann, G., Heckman, T. M., White, S. D. M., et al. 2003, MNRAS, 341, 54

Kennicutt, R. C., Jr. 1989, ApJ, 344, 685

Kennicutt, R. C., Jr. 1998a, ApJ, 498, 541

Kennicutt, R. C., Jr. 1998b, ARA\&A, 36, 189

Kennicutt, R. C., Jr., Armus, L., Bendo, G., et al. 2003, PASP, 115, 928

Kennicutt, R. C., Jr., Calzetti, D., Walter, F., et al. 2007, ApJ, 671, 333

Kennicutt, R. C., Jr., Hao, C.-N., Calzetti, D., et al. 2009, ApJ, 703, 1672

Kennicutt, R. C., Calzetti, D., Aniano, G., et al. 2011, PASP, 123, 1347

Kewley, L. J., \& Ellison, S. L. 2008, ApJ, 681, 1183

Köhler, M., Jones, A., \& Ysard, N. 2014, A\&A, 565, L9

Köhler, M., Ysard, N., \& Jones, A. P. 2015, A\&A, 579, A15

Krumholz, M. R. 2011, AIP Conf. Ser., 1386, 9

Kuno, N., Sato, N., Nakanishi, H., et al. 2007, PASJ, 59, 117

Law, K.-H., Gordon, K. D., \& Misselt, K. A. 2011, ApJ, 738, 124

Leitherer, C., Schaerer, D., Goldader, J. D., et al. 1999, ApJS, 123, 3

Leroy, A. K., Walter, F., Brinks, E., et al. 2008, AJ, 136, 2782

Leroy, A. K., Walter, F., Bigiel, F., et al. 2009, AJ, 137, 4670

Leroy, A. K., Bigiel, F., de Blok, W. J. G., et al. 2012, AJ, 144, 3

Lisenfeld, U., \& Ferrara, A. 1998, ApJ, 496, 145

López-Sánchez, Á. R., Dopita, M. A., Kewley, L. J., et al. 2012, MNRAS, 426, 2630

MacArthur, L. A., Courteau, S., \& Holtzman, J. A. 2003, ApJ, 582, 689

MacArthur, L. A., Courteau, S., Bell, E., \& Holtzman, J. A. 2004, ApJS, 152, 175

Magrini, L., Bianchi, S., Corbelli, E., et al. 2011, A\&A, 535, A13

Magrini, L., Coccato, L., Stanghellini, L., Casasola, V., \& Galli, D. 2016, A\&A, 588, A91

Marigo, P., Girardi, L., Bressan, A., et al. 2008, A\&A, 482, 883

Mathis, J. S., Mezger, P. G., \& Panagia, N. 1983, A\&A, 128, 212

Mao, Y.-W., Kennicutt, R. C., Jr., Hao, C.-N., Kong, X., \& Zhou, X. 2012, ApJ, 757, 52

Maraston, C. 2005, MNRAS, 362, 799

Mattsson, L., Gomez, H. L., Andersen, A. C., et al. 2014, MNRAS, 444, 797

McGaugh, S. S., \& Schombert, J. M. 2014, AJ, 148, 77

Meidt, S. E., Schinnerer, E., Knapen, J. H., et al. 2012, ApJ, 744, 17

Misiriotis, A., Xilouris, E. M., Papamastorakis, J., Boumis, P., \& Goudis, C. D. 2006, A\&A, 459, 113

Mollá, M., \& Díaz, A. I. 2005, MNRAS, 358, 521

Momose, R., Koda, J., Kennicutt, R. C., Jr., et al. 2013, ApJ, 772, L13

Moriondo, G., Giovanardi, C., \& Hunt, L. K. 1998, A\&AS, 130, 81

Möllenhoff, C. 2004, A\&A, 415, 63

Möllenhoff, C., Popescu, C. C., \& Tuffs, R. J. 2006, A\&A, 456, 941

Morrissey, P., Conrow, T., Barlow, T. A., et al. 2007, ApJS, 173, 682
Moustakas, J., Kennicutt, R. C., Jr., Tremonti, C. A., et al. 2010, ApJS, 190, 233 Muñoz-Mateos, J. C., Gil de Paz, A., Boissier, S., et al. 2007, ApJ, 658, 1006 Muñoz-Mateos, J. C., Gil de Paz, A., Boissier, S., et al. 2009, ApJ, 701, 1965 Muñoz-Mateos, J. C., Boissier, S., Gil de Paz, A., et al. 2011, ApJ, 731, 10

Narayanan, D., Krumholz, M. R., Ostriker, E. C., \& Hernquist, L. 2012, MNRAS, 421, 3127

Nguyen, H. T., Schulz, B., Levenson, L., et al. 2010, A\&A, 518, L5

Nieten, C., Neininger, N., Guélin, M., et al. 2006, A\&A, 453, 459

Ondrechen, M. P., van der Hulst, J. M., \& Hummel, E. 1989, ApJ, 342, 39

Osterbrock, D. E. 1981, ApJ, 249, 462

Peletier, R. F., Valentijn, E. A., Moorwood, A. F. M., \& Freudling, W. 1994, A\&AS, 108,

Pérez-González, P. G., Gil de Paz, A., Zamorano, J., et al. 2003, MNRAS, 338, 525

Pérez-González, P. G., Kennicutt, R. C., Jr., Gordon, K. D., et al. 2006, ApJ, 648, 987

Peters, S. P. C., van der Kruit, P. C., \& de Jong, R. S. 2017, MNRAS, 464, 1591 Pilyugin, L. S., Grebel, E. K., Zinchenko, I. A., \& Kniazev, A. Y. 2014, AJ, 148, 134

Piovan, L., Tantalo, R., \& Chiosi, C. 2006, MNRAS, 366, 923

Pohlen, M., Cortese, L., Smith, M. W. L., et al. 2010, A\&A, 518, L72

Pompei, E., \& Natali, G. 1997, A\&AS, 124,

Popescu, C. C., \& Tuffs, R. J. 2003, A\&A, 410, L21

Querejeta, M., Meidt, S. E., Schinnerer, E., et al. 2015, ApJS, 219, 5

Rahman, N., Bolatto, A. D., Wong, T., et al. 2011, ApJ, 730, 72

Regan, M. W., Thornley, M. D., Helfer, T. T., et al. 2001, ApJ, 561, 218

Regan, M. W., Thornley, M. D., Vogel, S. N., et al. 2006, ApJ, 652, 1112

Rémy-Ruyer, A., Madden, S. C., Galliano, F., et al. 2013, A\&A, 557, A95

Rémy-Ruyer, A., Madden, S. C., Galliano, F., et al. 2014, A\&A, 563, A31

Rémy-Ruyer, A., Madden, S. C., Galliano, F., et al. 2015, A\&A, 582, A121

Salim, S., Rich, R. M., Charlot, S., et al. 2007, ApJS, 173, 267

Salpeter, E. E. 1955, ApJ, 121, 161

Sandstrom, K. M., Leroy, A. K., Walter, F., et al. 2013, ApJ, 777, 5

Sauvage, M., Tuffs, R. J., \& Popescu, C. C. 2005, Space Sci. Rev., 119, 313

Simonian, G. V., \& Martini, P. 2017, MNRAS, 464, 3920

Schruba, A., Leroy, A. K., Walter, F., et al. 2011, AJ, 142, 37

Scodeggio, M., Gavazzi, G., Franzetti, P., et al. 2002, A\&A, 384, 812

Scoville, N. Z., Yun, M. S., Sanders, D. B., Clemens, D. P., \& Waller, W. H. 1987, ApJS, 63, 821

Smith, M. W. L., Gomez, H. L., Eales, S. A., et al. 2012a, ApJ, 748, 123

Smith, M. W. L., Eales, S. A., Gomez, H. L., et al. 2012b, ApJ, 756, 40

Smith, M. W. L., Eales, S. A., De Looze, I., et al. 2016, MNRAS, 462, 331

Solomon, P. M., Rivolo, A. R., Barrett, J., \& Yahil, A. 1987, ApJ, 319, 730

Strong, A. W., \& Mattox, J. R. 1996, A\&A, 308, L21

Swaters, R. A., van Albada, T. S., van der Hulst, J. M., \& Sancisi, R. 2002, A\&A, 390,829

Tabatabaei, F. S., Braine, J., Xilouris, E. M., et al. 2014, A\&A, 561, A95

Taylor, V. A., Jansen, R. A., Windhorst, R. A., Odewahn, S. C., \& Hibbard, J. E. 2005, ApJ, 630, 784

Trayford, J. W., Theuns, T., Bower, R. G., et al. 2016, MNRAS, 460, 3925

Tully, R. B. 1988, Nearby galaxies catalog (Cambridge and New York: Cambridge University Press)

Valiante, R., Schneider, R., Bianchi, S., \& Andersen, A. C. 2009, MNRAS, 397, 1661

van der Kruit, P. C., \& Freeman, K. C. 2011, ARA\&A, 49, 301

Veilleux, S., Cecil, G., \& Bland-Hawthorn, J. 2005, ARA\&A, 43, 769

Villaume, A., Conroy, C., \& Johnson, B. D. 2015, ApJ, 806, 82

Walter, F., Brinks, E., de Blok, W. J. G., et al. 2008, AJ, 136, 2563

Weiß, A., Neininger, N., Hüttemeister, S., \& Klein, U. 2001, A\&A, 365, 571

Westmeier, T., Braun, R., \& Koribalski, B. S. 2011, MNRAS, 410, 2217

Willner, S. P., Ashby, M. L. N., Barmby, P., et al. 2004, ApJS, 154, 222

Wilson, C. D. 1995, ApJ, 448, L97

Wong, T., \& Blitz, L. 2002, ApJ, 569, 157

Wright, E. L., Eisenhardt, P. R. M., Mainzer, A. K., et al. 2010, AJ, 140, 1868

Xilouris, E. M., Byun, Y. I., Kylafis, N. D., Paleologou, E. V., \& Papamastorakis, J. 1999, A\&A, 344, 868

York, D. G., Adelman, J., Anderson, J. E., Jr., et al. 2000, AJ, 120, 1579

Ysard, N., Köhler, M., Jones, A., et al. 2015, A\&A, 577, A110

Zibetti, S., Charlot, S., \& Rix, H.-W. 2009, MNRAS, 400, 1181 


\section{Appendix A: Additional figures}
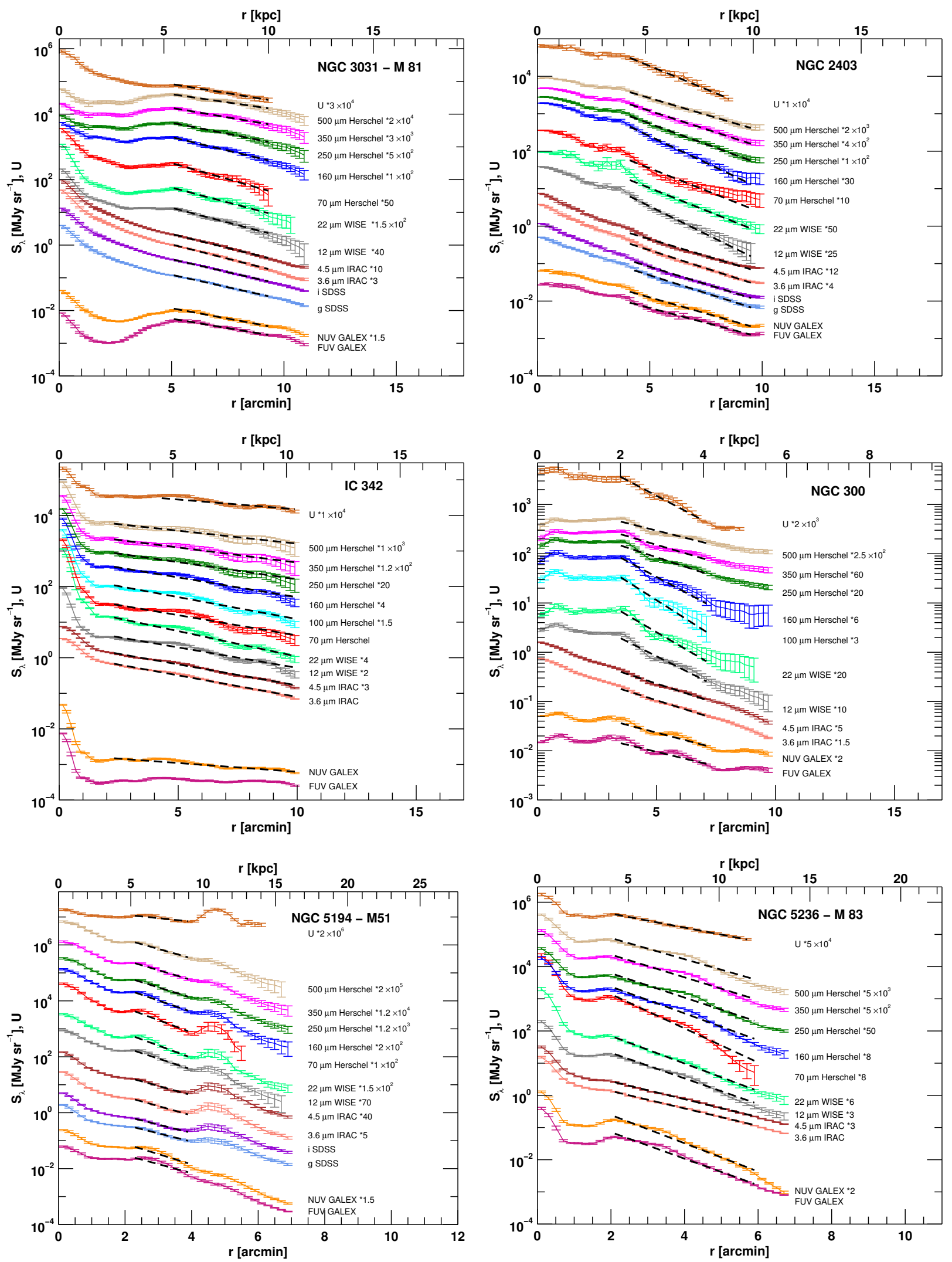

Fig. A.1. Multi-wavelength surface-brightness and $U$ profiles for the face-on DustPedia sample, except for the galaxy NGC 5457 (M 101) already displayed in Fig. 2, shown up to $r_{25}$. The profiles are shown as in Fig. 2. The black dashed lines are exponential fits performed avoiding the central part of galaxies up to the maximum extension of the $70 \mu \mathrm{m}$ profile (or the $12 \mu \mathrm{m}$ profile for NGC 6946 and the $100 \mu \mathrm{m}$ profiles for NGC 1097 and NGC 300). 
V. Casasola et al.: Scale-lengths in DustPedia
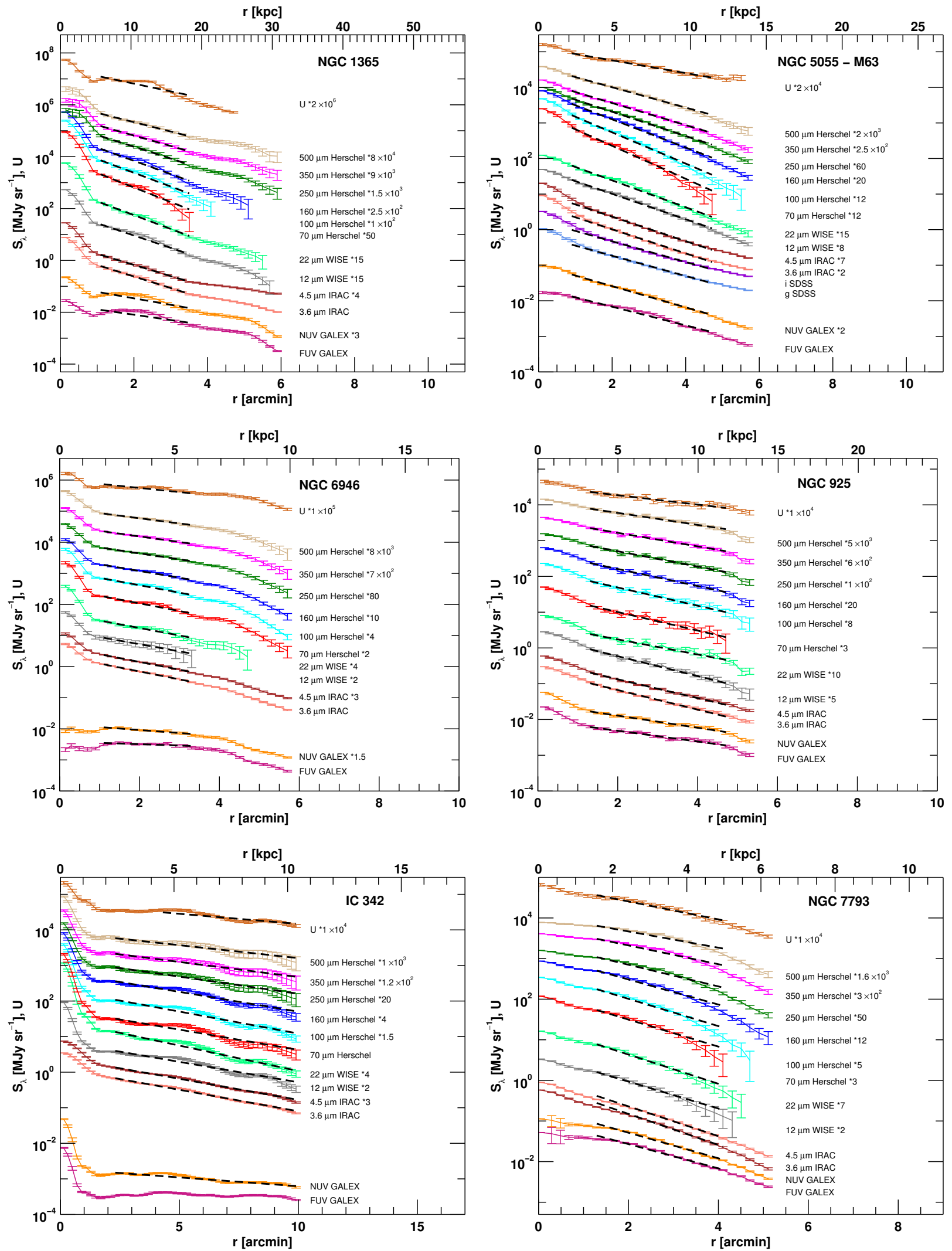

Fig. A.1. continued. 
A\&A 605, A18 (2017)
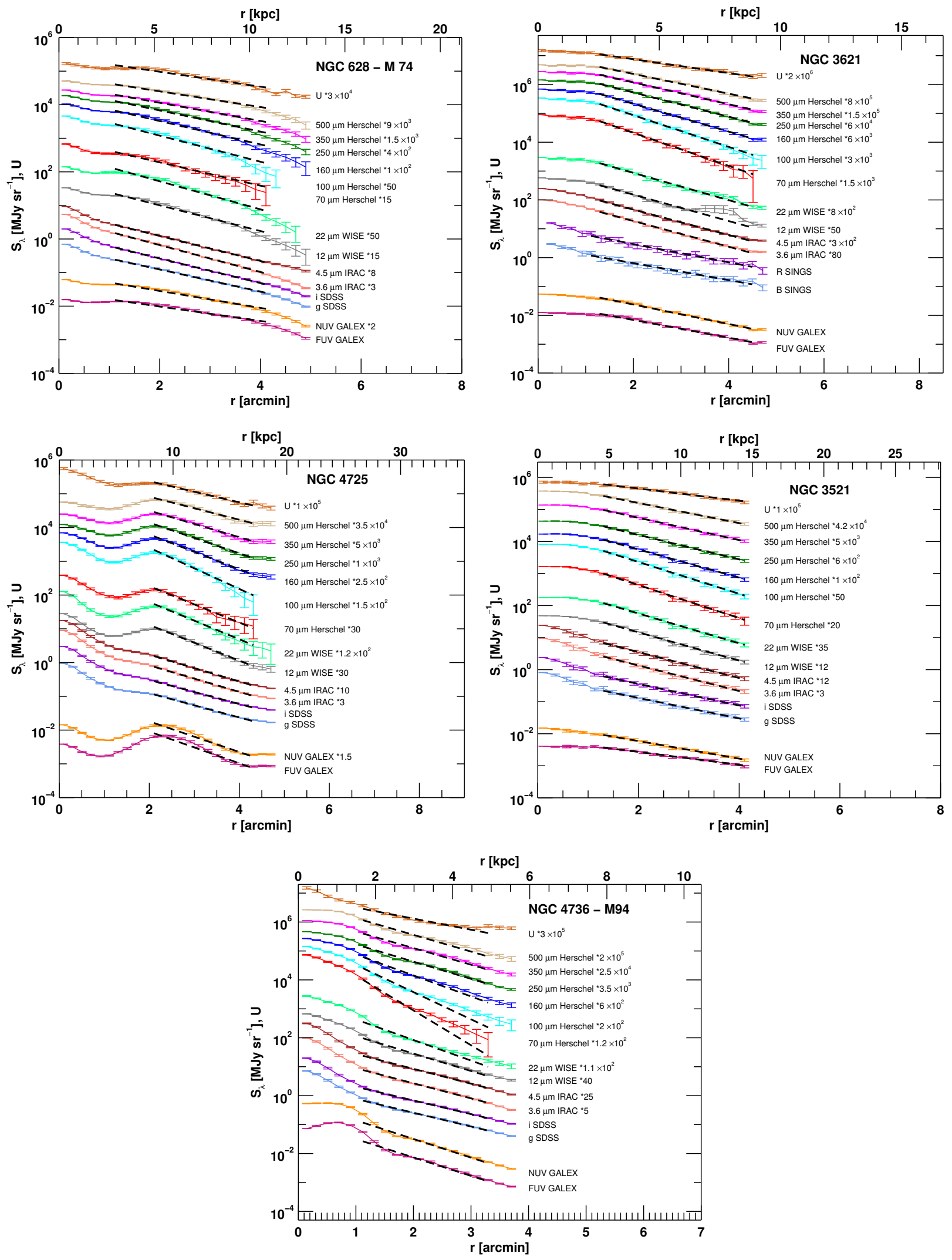

Fig. A.1. continued. 

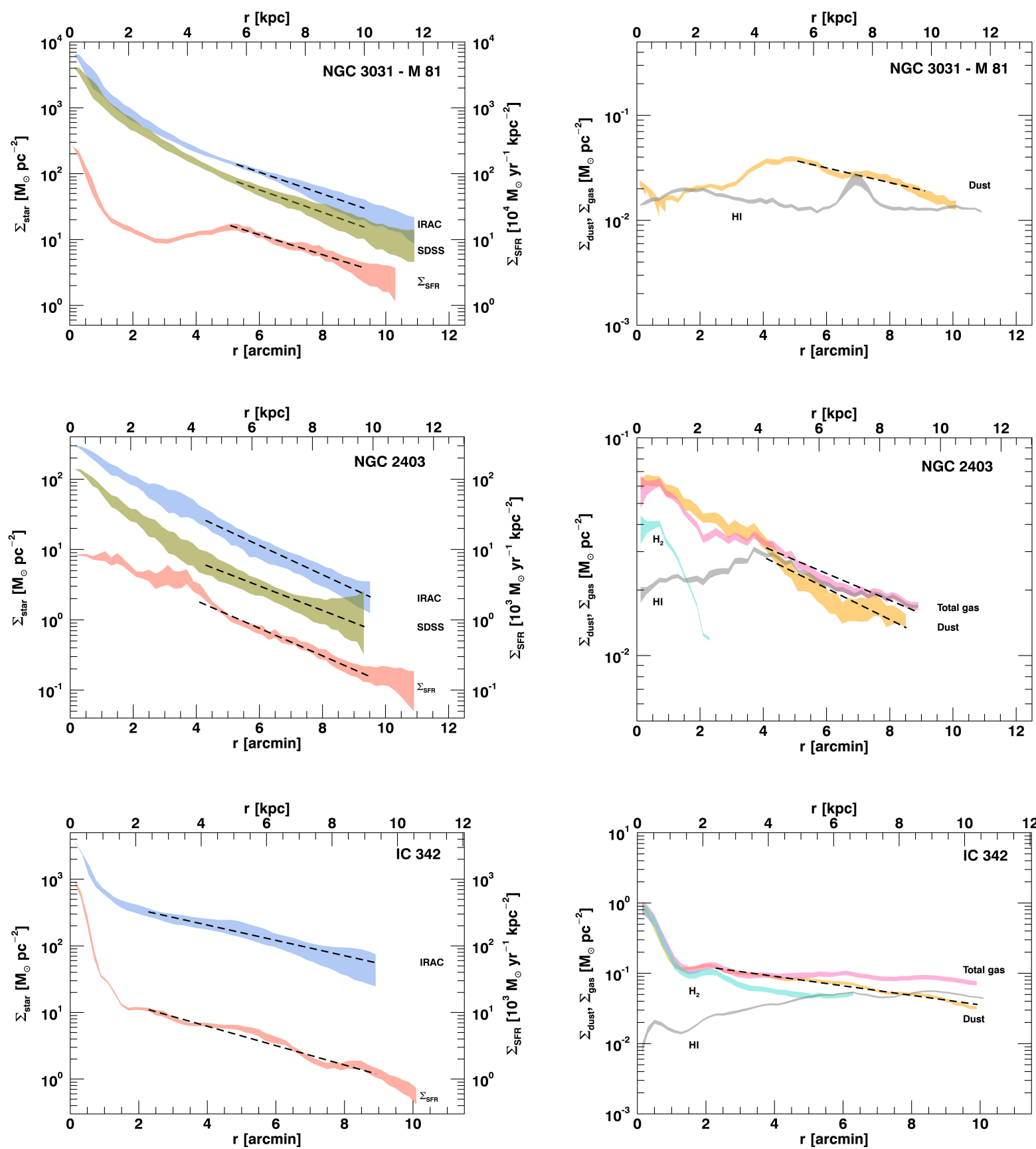

Fig. A.2. Left panel: stellar-mass surface-density profiles for the face-on DustPedia sample, except for the galaxy NGC 5457 (M 101) already displayed in the left panel of Fig. 2. The profiles are shown as in the left panel of Fig. 2. The black dashed lines are exponential fits performed avoiding the central part of galaxies in the same radius ranges of Fig. A.1 or up to the profile is available. For NGC 3621 and NGC 1097 stellarmass surface-density profiles derived from SINGS are plotted without error bars since these profiles have overly large uncertainties to be displayed in a logarithmic scale. Right panel: surface-density profiles for the mass of dust, molecular gas, atomic gas, and total gas for the entire DustPedia face-on sample, except for NGC 5457 (M 101) already shown in the right panel of Fig. 2. The profiles are shown as in the right panel of Fig. 2. 

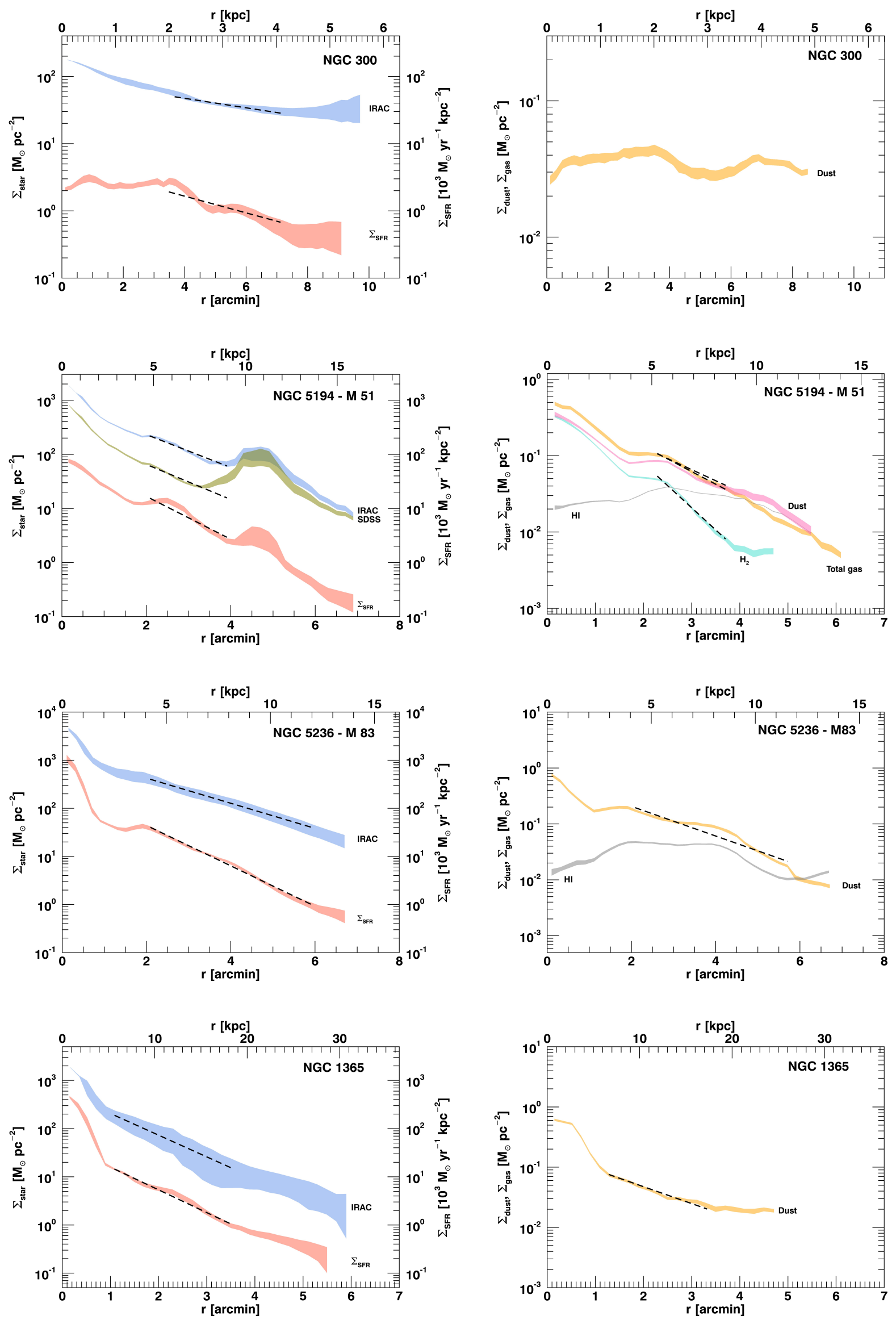

Fig. A.2. continued. 
V. Casasola et al.: Scale-lengths in DustPedia
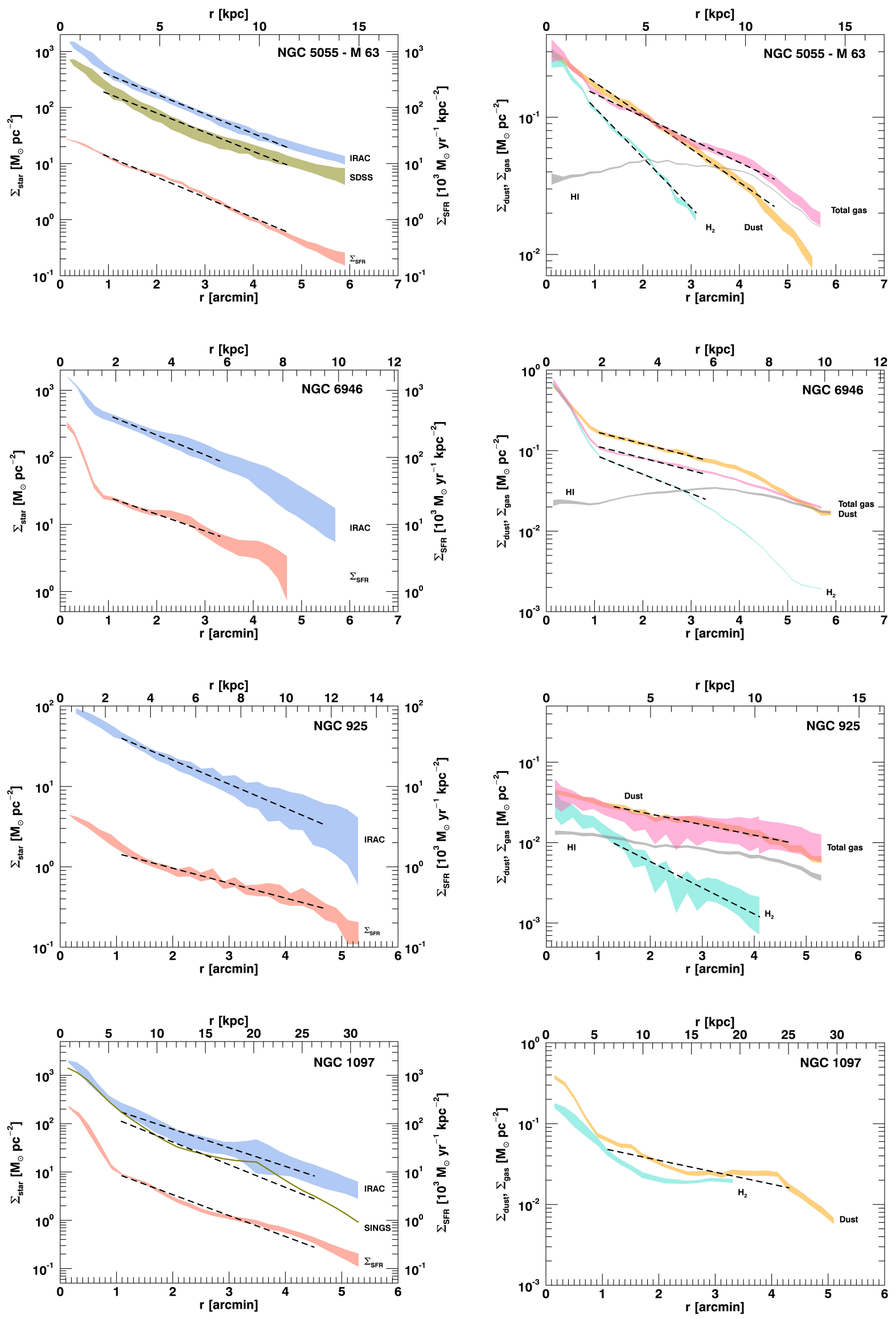

Fig. A.2. continued. 

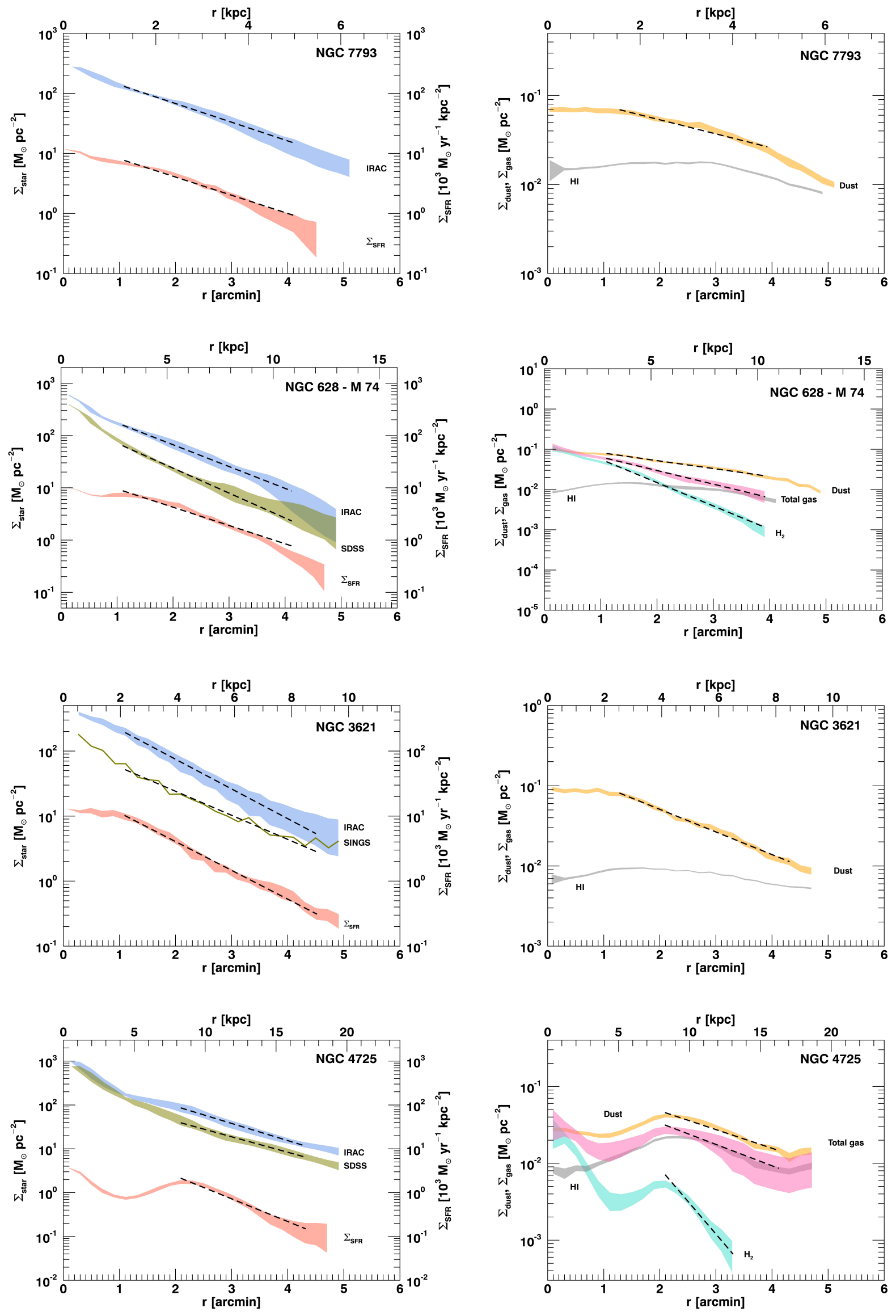

Fig. A.2. continued. 
V. Casasola et al.: Scale-lengths in DustPedia
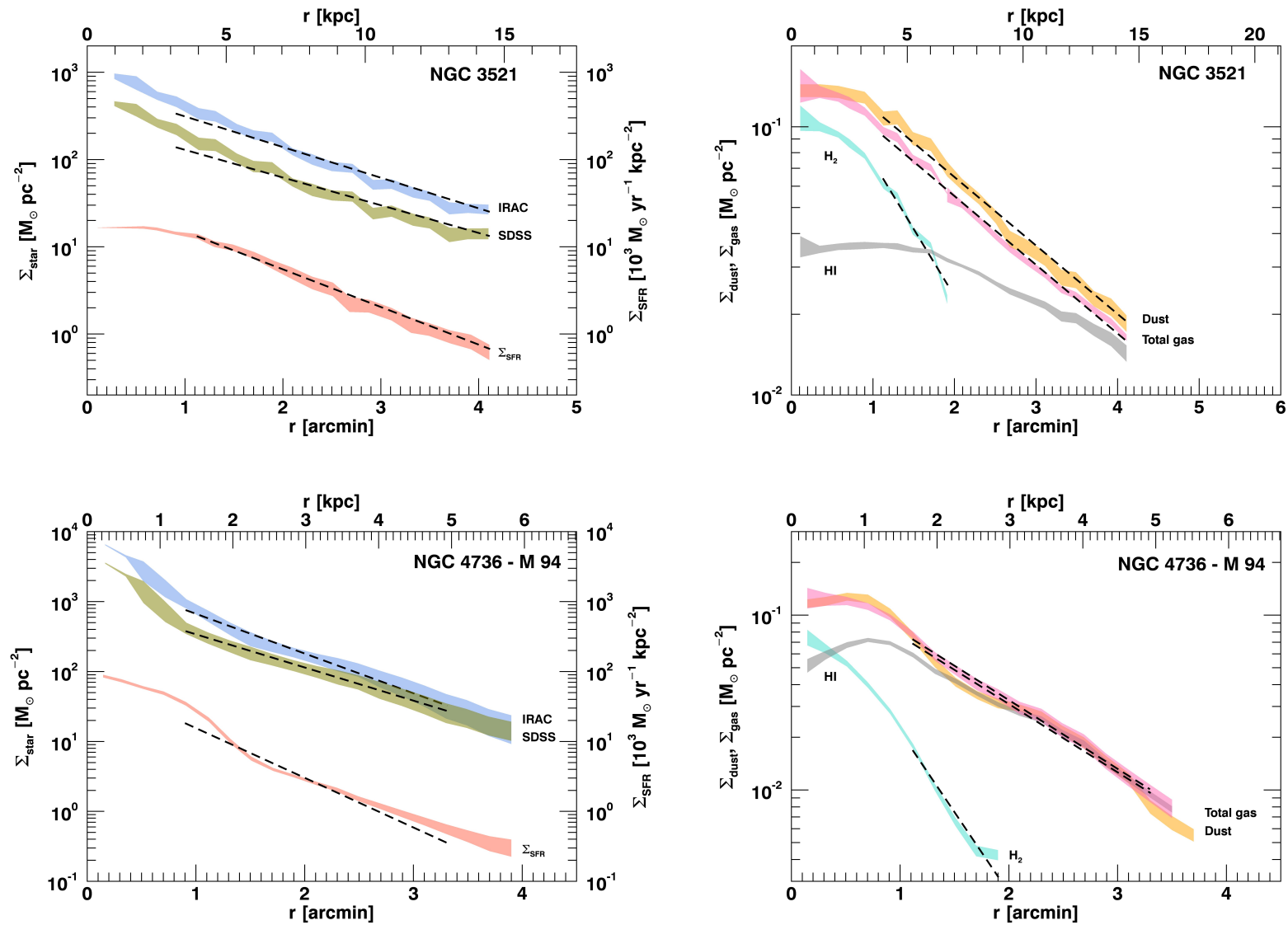

Fig. A.2. continued. 

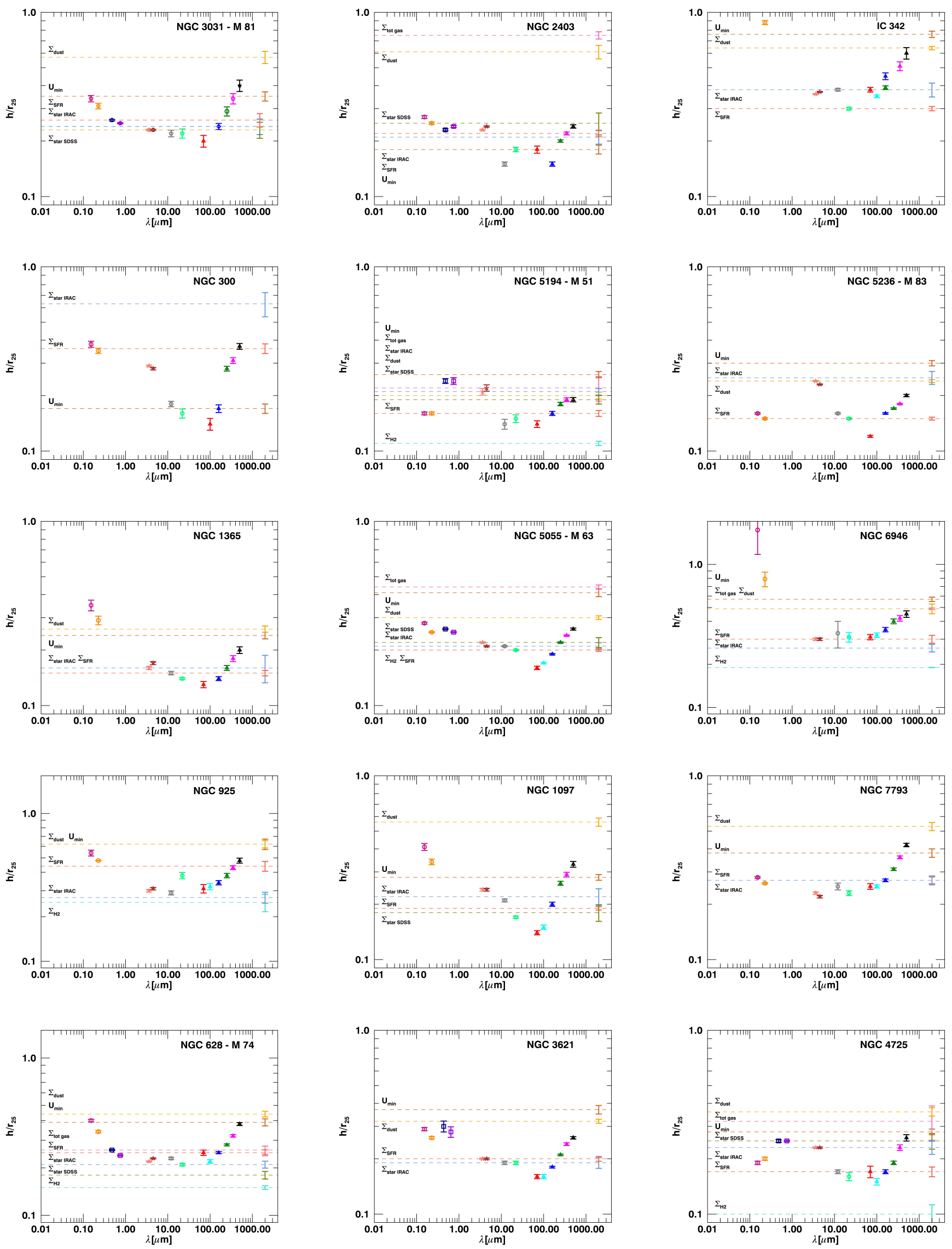

Fig. A.3. Scale-lengths of surface-brightness profiles from UV to sub-mm, normalized with respect $r_{25}$, as a function of wavelength for the face-on DustPedia sample, except for the galaxy NGC 5457 (M 101) already shown in Fig. 4. Scale-lengths of mass (of dust, gas, and stars) and SFR surface-density profiles, scale-length of $U$, and the corresponding error bars are drawn as in Fig. 4. 
V. Casasola et al.: Scale-lengths in DustPedia
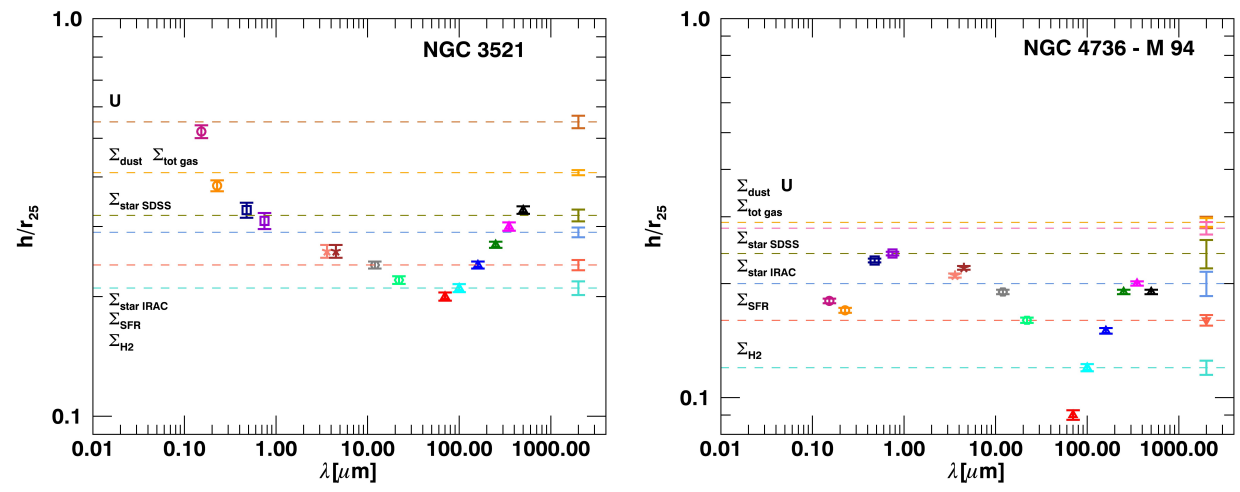

Fig. A.3. continued. 